Prepared in cooperation with the Bureau of Land Management, U.S. Department of the Interior

\title{
Rainfall-Runoff and Erosion Data from the Mancos Shale Formation in the Gunnison Gorge National Conservation Area, Southwestern Colorado, 2003-2006
}

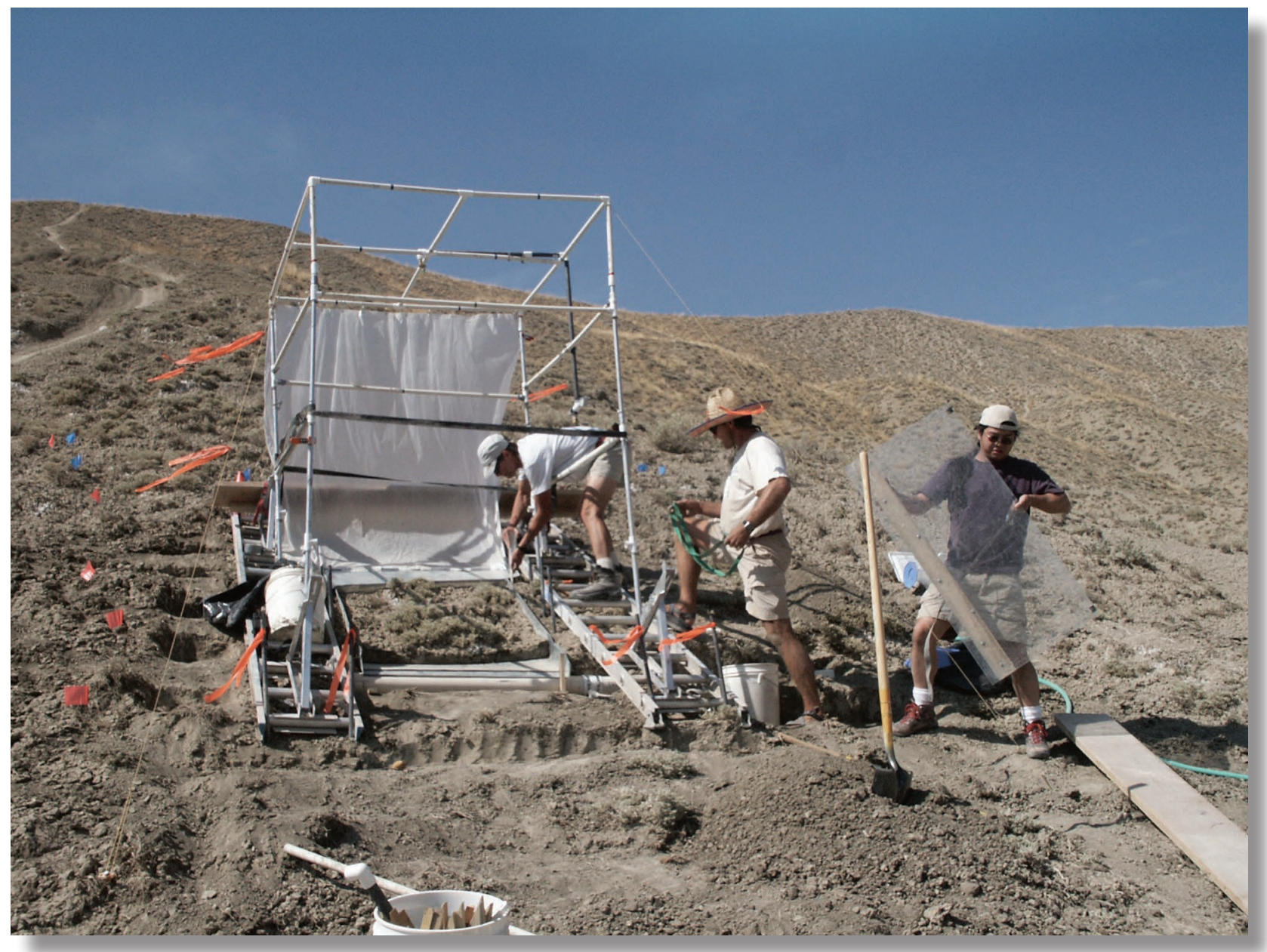

Open-File Report 2007-1002G 



\section{Rainfall-Runoff and Erosion Data from the Mancos Shale Formation in the Gunnison Gorge National Conservation Area, Southwestern Colorado, 2003-2006}

By John G. Elliott, James R. Herring, George P. Ingersoll, John J. Kosovich, and Juli Fahy

Prepared in cooperation with the Bureau of Land Management, U.S. Department of the Interior

Open-File Report 2007-1002G

U.S. Department of the Interior U.S. Geological Survey 


\title{
U.S. Department of the Interior DIRK KEMPTHORNE, Secretary
}

\author{
U.S. Geological Survey \\ Mark D. Myers, Director
}

U.S. Geological Survey, Reston, Virginia: 2008

\author{
About USGS Products \\ For product and ordering information: \\ World Wide Web: http://www.usgs.gov/pubprod \\ Telephone: 1-888-ASK-USGS \\ For more information on the USGS - the Federal source for science about the Earth, its natural and \\ living resources, natural hazards, and the environment: \\ World Wide Web: http://www.usgs.gov \\ Telephone: 1-888-ASK-USGS \\ Publishing support provided by: \\ Denver Publishing Service Center, Denver, Colorado \\ Manuscript approved for publication, September 2007 \\ Edited by Lanna Combs and Jon Raese \\ Suggested citation: \\ Elliott, J.G., Herring, J.R., Ingersoll, G.P., Kosovich, J.J., and Fahy, Juli, 2008, Rainfall-runoff and erosion \\ data from the Mancos Shale Formation in the Gunnison Gorge National Conservation Area, southwestern \\ Colorado, 2003-2006: U.S. Geological Survey Open-File Report 2007-1002G, 67 p. \\ Any use of trade, product, or firm names is for descriptive purposes only and does not imply endorsement \\ by the U.S. Government. \\ Although this report is in the public domain, permission must be secured from the individual copyright \\ owners to reproduce any copyrighted materials contained within this report.
}




\section{Contents}

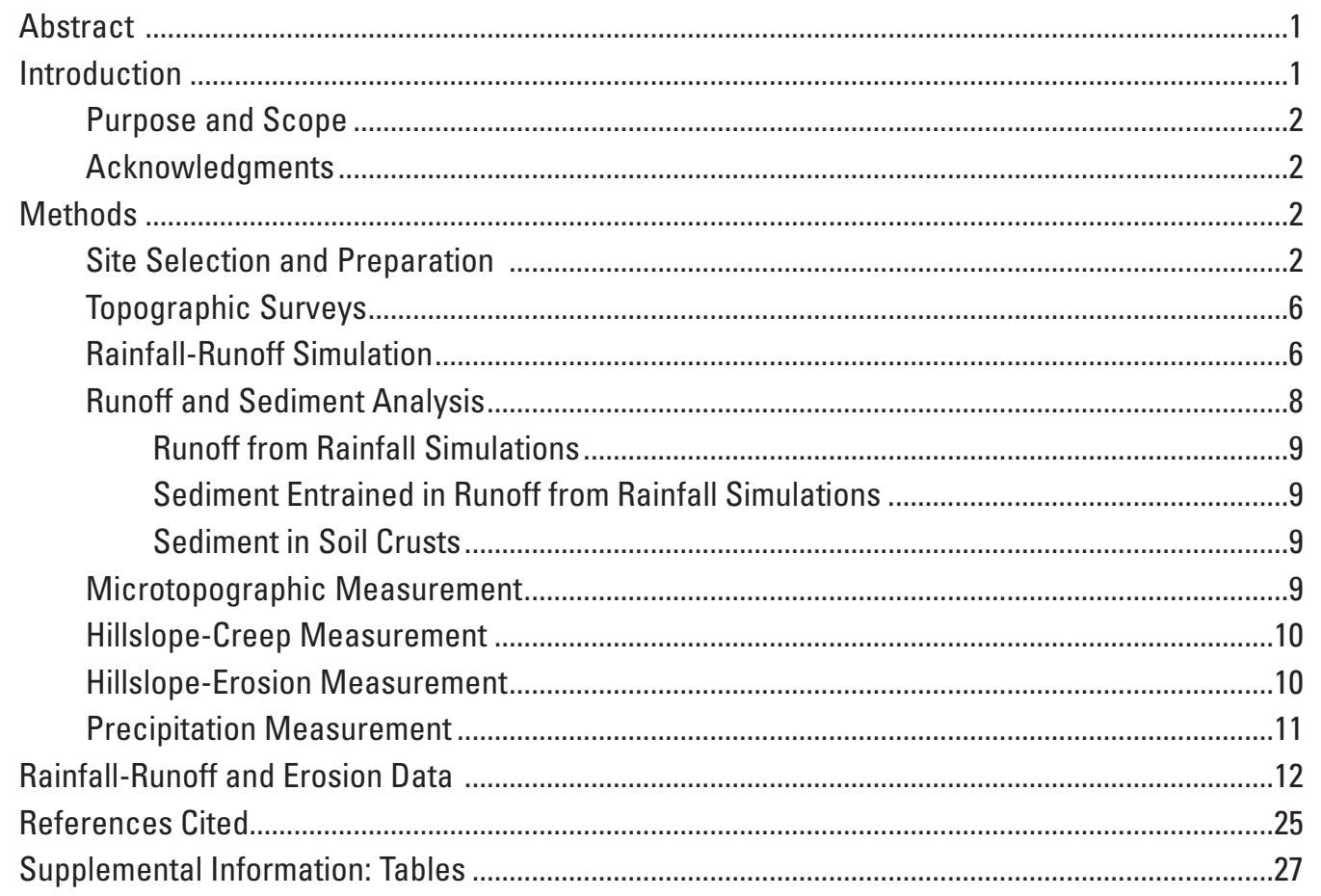

\section{Figures}

1. Map showing Gunnison Gorge National Conservation Area and the upper Elephant Skin Wash and Candy Lane study areas.......

2-4. LIDAR images showing location of:

2. Sampling sites in the upper Elephant Skin Wash study area........................................4

3. Sampling sites in the Candy Lane study area ....................................................................5

4. Arroyo headcut in the Candy Lane study area .................................................................

5-7. Photographs showing:

5. Simulated rainfall-runoff device set up on 1-square meter plot....................................8

6. Erosion bridge and microtopographic measurement...................................................10

7. Silt fence at a hillslope-erosion measurement site ......................................................11

8-12. Graphs showing:

8. Graph showing surveyed hillslope profile and geomorphic terminology at CP.8W...12

9. Total-runoff hydrographs from rainfall simulations......................................................14

10. Sediment-concentration hydrographs from rainfall simulations................................19

11. Soil-surface microtopography before and after a rainfall simulation, ES.9SE_U......24

12. Cumulative precipitation at Montrose \#2 climate station, upper Elephant

Skin Wash, and Candy Lane precipitation gages. 


\section{Tables}

1. Sampling-site locations in upper Elephant Skin Wash study area, Gunnison

Gorge National Conservation Area, southwestern Colorado.

2. Sampling-site locations in Candy Lane study area, Gunnison Gorge

National Conservation Area, southwestern Colorado

3. Rainfall-runoff and ancillary data from Gunnison Gorge National

Conservation Area, southwestern Colorado.

4. Rainfall-runoff and ancillary data from Gunnison Gorge National

Conservation Area, southwestern Colorado.

5. Hillslope-creep rates and ancillary data from Gunnison Gorge

National Conservation Area, southwestern Colorado.

6. Hillslope-erosion data from silt-fence traps on undisturbed areas at Gunnison Gorge National Conservation Area, southwestern Colorado.

7. Daily and cumulative precipitation at the Montrose \#2 climate station, upper Elephant Skin Wash, and Candy Lane rain gages, southwestern Colorado. 


\section{Conversion Factors, Abbreviations, and Datums}

\begin{tabular}{lcl}
\hline Multiply & \multicolumn{1}{c}{ By } & \multicolumn{1}{c}{ To obtain } \\
\hline millimeter $(\mathrm{mm})$ & 0.03937 & inch (in.) \\
meter $(\mathrm{m})$ & 3.281 & foot $(\mathrm{ft})$ \\
kilometer $(\mathrm{km})$ & 0.6214 & mile (mi) \\
square meter $\left(\mathrm{m}^{2}\right)$ & 10.76 & square foot $\left(\mathrm{ft}^{2}\right)$ \\
hectare $(\mathrm{ha})$ & 2.471 & acre \\
square kilometer $\left(\mathrm{km}^{2}\right)$ & 247.1 & acre \\
hectare $($ ha) & 0.003861 & square mile $\left(\mathrm{mi}^{2}\right)$ \\
square kilometer $\left(\mathrm{km}^{2}\right)$ & 0.3861 & square mile $\left(\mathrm{mi}^{2}\right)$ \\
liter $(\mathrm{L})$ & 33.82 & ounce, fluid $(\mathrm{fl} . \mathrm{oz})$ \\
liter $(\mathrm{L})$ & 0.2642 & gallon $(\mathrm{gal})$ \\
liter $(\mathrm{L})$ & 61.02 & cubic inch $\left(\mathrm{in}{ }^{3}\right)$ \\
cubic meter $\left(\mathrm{m}^{3}\right)$ & 35.31 & cubic foot $\left(\mathrm{ft}^{3}\right)$ \\
gram $(\mathrm{g})$ & 0.03527 & ounce, avoirdupois $(\mathrm{oz})$ \\
kilogram $(\mathrm{kg})$ & 2.205 & pound avoirdupois $(\mathrm{lb})$ \\
megagram $(\mathrm{Mg})$ & 1.102 & ton, short $(2,000 \mathrm{lb})$ \\
kilopascal $(\mathrm{kPa})$ & 0.1450 & pound per square inch $\left(\mathrm{lb} / \mathrm{in}^{2}\right)$ \\
inch (in.) & 25.4 & millimeter $(\mathrm{mm})$ \\
gallon $(\mathrm{gal})$ & 3.785 & liter $(\mathrm{L})$ \\
gallon per minute $(\mathrm{gal} / \mathrm{min})$ & 0.06309 & liter per second $(\mathrm{L} / \mathrm{s})$ \\
pound per square inch $\left(\mathrm{lb} / \mathrm{in}^{2}\right)$ & 6.895 & kilopascal $(\mathrm{kPa})$ \\
\hline
\end{tabular}

Temperature in degrees Celsius $\left({ }^{\circ} \mathrm{C}\right)$ may be converted to degrees Fahrenheit $\left({ }^{\circ} \mathrm{F}\right)$ as follows:

$$
{ }^{\circ} \mathrm{F}=\left(1.8 x^{\circ} \mathrm{C}\right)+32
$$

Temperature in degrees Fahrenheit $\left({ }^{\circ} \mathrm{F}\right)$ may be converted to degrees Celsius $\left({ }^{\circ} \mathrm{C}\right)$ as follows:

$$
{ }^{\circ} \mathrm{C}=\left({ }^{\circ} \mathrm{F}-32\right) / 1.8
$$

Vertical coordinate information is referenced to the North American Vertical Datum of 1988 (NAVD 88).

Horizontal coordinate information is referenced to the North American Datum of 1983 (NAD 83).

Altitude, as used in this report, refers to distance above the vertical datum. 


\title{
Rainfall-Runoff and Erosion Data from the Mancos Shale Formation in the Gunnison Gorge National Conservation Area, Southwestern Colorado, 2003-2006
}

\author{
By John G. Elliott' ${ }^{1}$, James R. Herring ${ }^{2}$, George P. Ingersoll ${ }^{1}$, John Kosovich ${ }^{3}$, and Juli Fahy ${ }^{4}$
}

\begin{abstract}
Data were collected and experiments were conducted from 2003 to 2006 by the U.S. Geological Survey, in cooperation with the Bureau of Land Management, to support research into understanding processes that liberate, disperse, and concentrate erosion byproducts in Mancos Shale landscapes. The study area was the Gunnison Gorge National Conservation Area near Montrose and Delta, Colorado. This report includes data collected from 24, small-plot, rainfall-runoff simulations, 6 hillslope-erosion monitoring plots, 20 hillslope-creep monitoring sites, and 3 precipitation gages. Small-plot rainfall-runoff simulations were performed on paired (undisturbed and disturbed) plots to examine the effect of off-highway vehicle use on runoff and erosion. These data were collected in conjunction with several other studies done by the U.S. Geological Survey in the Gunnison Gorge National Conservation Area. Data collected in companion studies are published in separate open-file reports.
\end{abstract}

\section{Introduction}

Mancos Shale landscapes of the Western United States, especially in the upper Colorado River Basin, have become a focal point for the need for science information that will support sound land-use policy development. This need has become more prominent primarily because of (1) increased and changing demands for land use and (2) issues related to selenium, salinity, and sediment. Also, there is an increased awareness within the scientific community of the need to better understand processes leading to the concentration or dispersal of both economically important and environmentally sensitive elements in widely dispersed black shale regions, including the Mancos Shale in southwestern Colorado.

An understanding of the processes leading to the concentration of organic matter, elements of economic importance, and environmentally sensitive elements is fundamental to developing predictive models that can be used to evaluate black shale landscapes for their mineral resource potential and their environmental sensitivity. In many instances, geomorphic, geologic, hydrologic, biologic, and land-use data are insufficient for resource and land managers to formulate scientifically supportable policies for (1) the sustainable development of mineral and energy resources contained in black shale terrains and (2) the multiple uses of public resources in black shale landscapes. There is a lack of detailed understanding of the relations and processes required to create predictive models that can effectively take into account changes in land use, climate, national resource need, or a variety of other factors.

The study was done in cooperation with the Bureau of Land Management in the Gunnison Gorge National Conservation Area (GGNCA) near Montrose and Delta, Colorado (fig. 1). The Mancos Shale in GGNCA outcrops between the southern and western rims of the Black Canyon and Gunnison Gorge and, beyond the GGNCA boundary, extends westward into the Uncompahgre River valley (Kellogg, 2004). Climate of the study area is semiarid, with a mean maximum annual temperature of $17.4^{\circ} \mathrm{C}\left(63.3^{\circ} \mathrm{F}\right)$, a mean minimum annual temperature of $1.4^{\circ} \mathrm{C}\left(34.6^{\circ} \mathrm{F}\right)$, and mean annual precipitation of $242 \mathrm{~mm}$ (9.53 in.) at Montrose (National Oceanic and Atmospheric Administration, 2007). Forty-four percent of the mean annual precipitation occurs as rain in July through October, but measurable snow can occur from October through April. The 2-year, 1-hour rainfall total is $15 \mathrm{~mm}$ (0.59 in.) and the 100-year, 1-hour rainfall total is $38 \mathrm{~mm}$ (1.51 in.) (Miller and others, 1973).

${ }^{1}$ U.S. Geological Survey, Box 25046, MS 415, DFC, Lakewood, CO 80225.

${ }^{2}$ U.S. Geological Survey, Box 25046, MS 973, DFC, Lakewood, CO 80225.

${ }^{3}$ U.S. Geological Survey, Box 25046, MS 516, DFC, Lakewood, CO 80225.

${ }^{4}$ Bureau of Reclamation, Box 25007, MS 86-68520, Lakewood, CO 80225. 
The study sites were located in the area of exposed Mancos Shale in GGNCA. Data were collected from small-plot rainfallrunoff simulations, hillslope-erosion monitoring plots, hillslope-creep measurements, and precipitation gages from 2003 to 2006 to support research into understanding processes that liberate, disperse, and concentrate erosion byproducts in Mancos Shale landscapes.

\section{Purpose and Scope}

The purpose of this report is to present data from several measurements and onsite simulations done as a part of this study. The report includes data collected from 2003 to 2006 from small-plot rainfall-runoff simulations, hillslope-erosion monitoring plots, hillslope-creep measurements, and precipitation gages located in the Gunnison Gorge National Conservation Area (GGNCA) near Montrose and Delta, Colorado (fig. 1).

\section{Acknowledgments}

The authors are grateful for the assistance received in collecting and processing the data presented in this report. Michael R. Stevens, Steven J. Char, Heather Sproule Eppler, Anthony J. Ranalli, Barbara C. Ruddy, and Paul von Guerard of the U.S. Geological Survey (USGS) provided assistance with rainfall-runoff simulations, hillslope creep measurements, and microtopographic measurements. James R. Herring, USGS (retired), collected simulated rain blanks and runoff samples for geochemical analyses. Mike Stevens and Steve Char also assisted with construction of silt fences used in the long-term, hillslope-erosion monitoring plots and with topographic surveying. Heather Sproule Eppler processed runoff and sediment samples from the rainfall simulations. Dave Catts, formerly of the USGS, made reconnaissance maps and computed initial geomorphic characteristics of the GGNCA.

Dennis Murphy, Bureau of Land Management (BLM), Montrose, assisted with installation and maintenance of the rain gages in GGNCA and provided earlier precipitation data from a nearby gage. Karen Tucker, BLM, Montrose, arranged for access to GGNCA through privately owned land. Glen Neigenfind, Clyde Waller, Al Griffin, Nora Hamilton, and Chad Greiner of BLM carefully disturbed the $1-\mathrm{m}^{2}$ treatment plots with their motorcycles before the rainfall-runoff simulations on disturbed soils. Marc Catlin, Uncompahgre Valley Water Users Canal, graciously provided a source of water for the rainfall simulations. Timothy Ward, University of New Mexico (Albuquerque), and Bruce Byars, Baylor University Center for Applied Geography and Spatial Research (Waco, Tex.), provided helpful discussions in the design modification and operation of the rainfall simulator. John Moody, USGS, provided design assistance and materials for the rainfall-runoff collection device and for microtopographic measuring equipment. Richard Grauch and Michele Tuttle, USGS, provided technical insights regarding study site locations. Concurrent, co-located data collection and analyses were conducted by James Herring, Richard Grauch, Michele Tuttle, Geneva Chong, John Kosovich, and Eric Livo, USGS.

\section{Methods}

Quantification of a variety of geomorphic and hydrologic processes in the Gunnison Gorge National Conservation Area (GGNCA) required several types of measurements, surveys, simulations, and instrumentation. Data from this study are presented in a "Supplemental Information" section at the end of this report. A co-located study of Mancos Shale rock material and shalederived soil is published in a companion report by Tuttle and others (2007). Other co-located studies included the geochemistry of the simulated rainfall runoff and sediment (J.R. Herring, U.S. Geological Survey, oral commun., 2003-2006), Mancos Shale geology (R.I. Grauch, U.S. Geological Survey, oral commun., 2003-2006), vegetation and biological soil crusts (G.W. Chong, U.S. Geological Survey, oral commun., 2003-2006), and geospatial data (J.J. Kosovich, U.S. Geological Survey, oral commun., 2004-2006).

\section{Site Selection and Preparation}

Study sites in the upper reaches of Elephant Skin Wash and on hillslopes in the Candy Lane area were chosen for sampling, monitoring, and experiments because of their relative remoteness and infrequent human visitation (figs. 2 and 3). Study sites were selected to represent a range of geomorphic settings in GGNCA with emphasis directed to characteristic areas where off-highway vehicular (OHV) use was a potential. Based on observations and BLM officials, steep hillslopes attract occasional to frequent motorcycle use in GGNCA and were presumed to be more affected by this type of land use than were low-gradient 


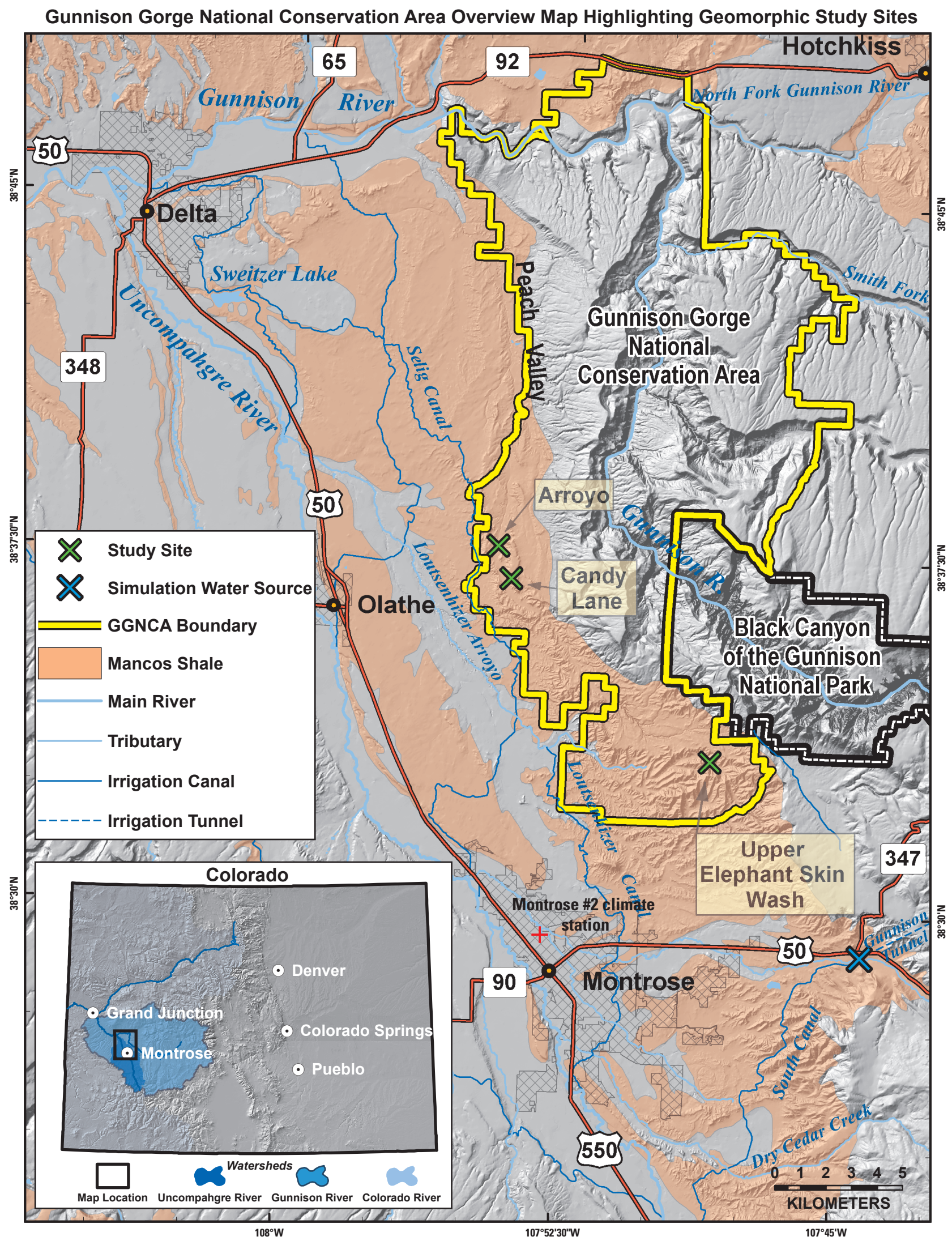

Figure 1. Gunnison Gorge National Conservation Area and the upper Elephant Skin Wash and Candy Lane study areas. 


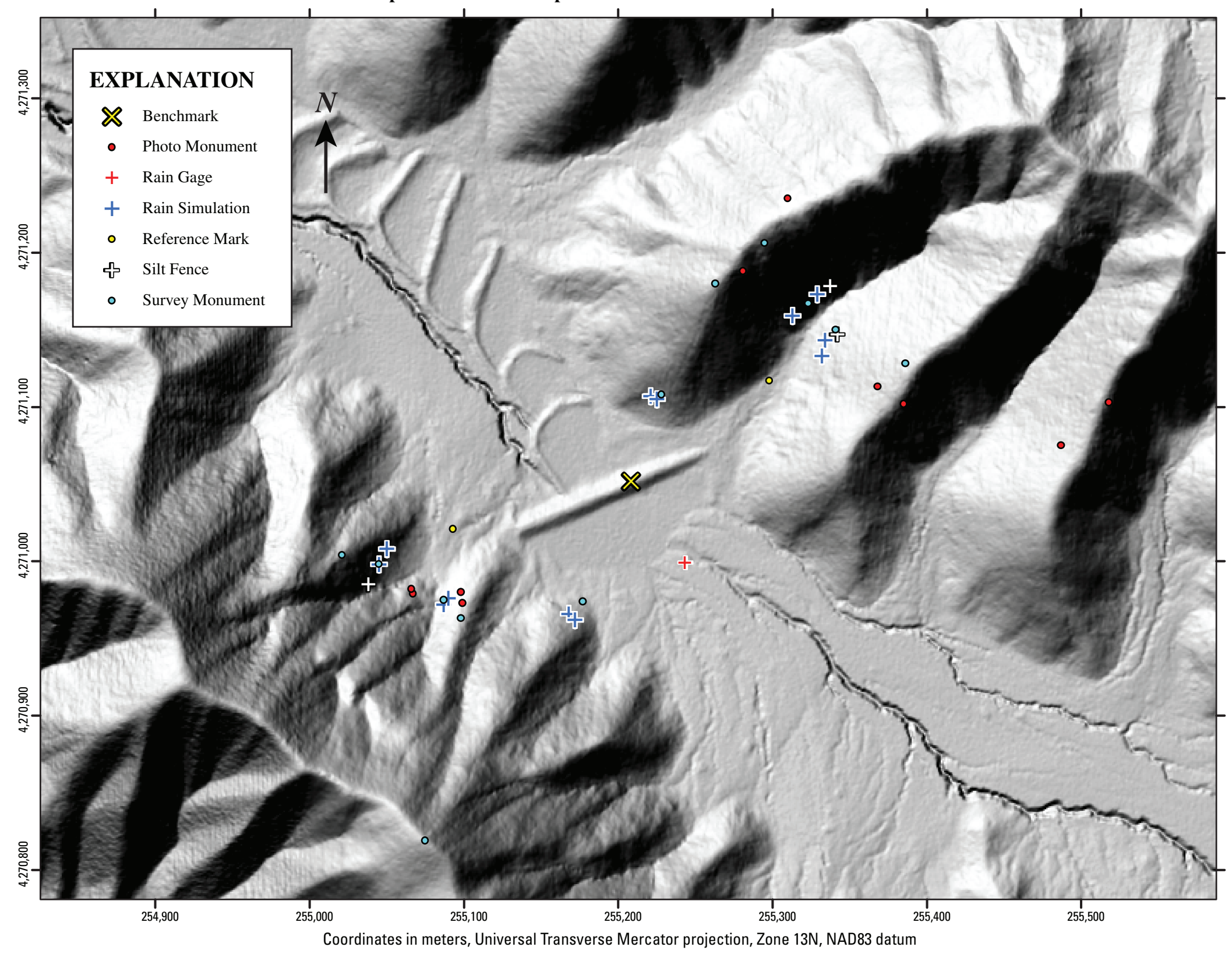

Figure 2. LIDAR image showing location of sampling sites in the upper Elephant Skin Wash study area. 


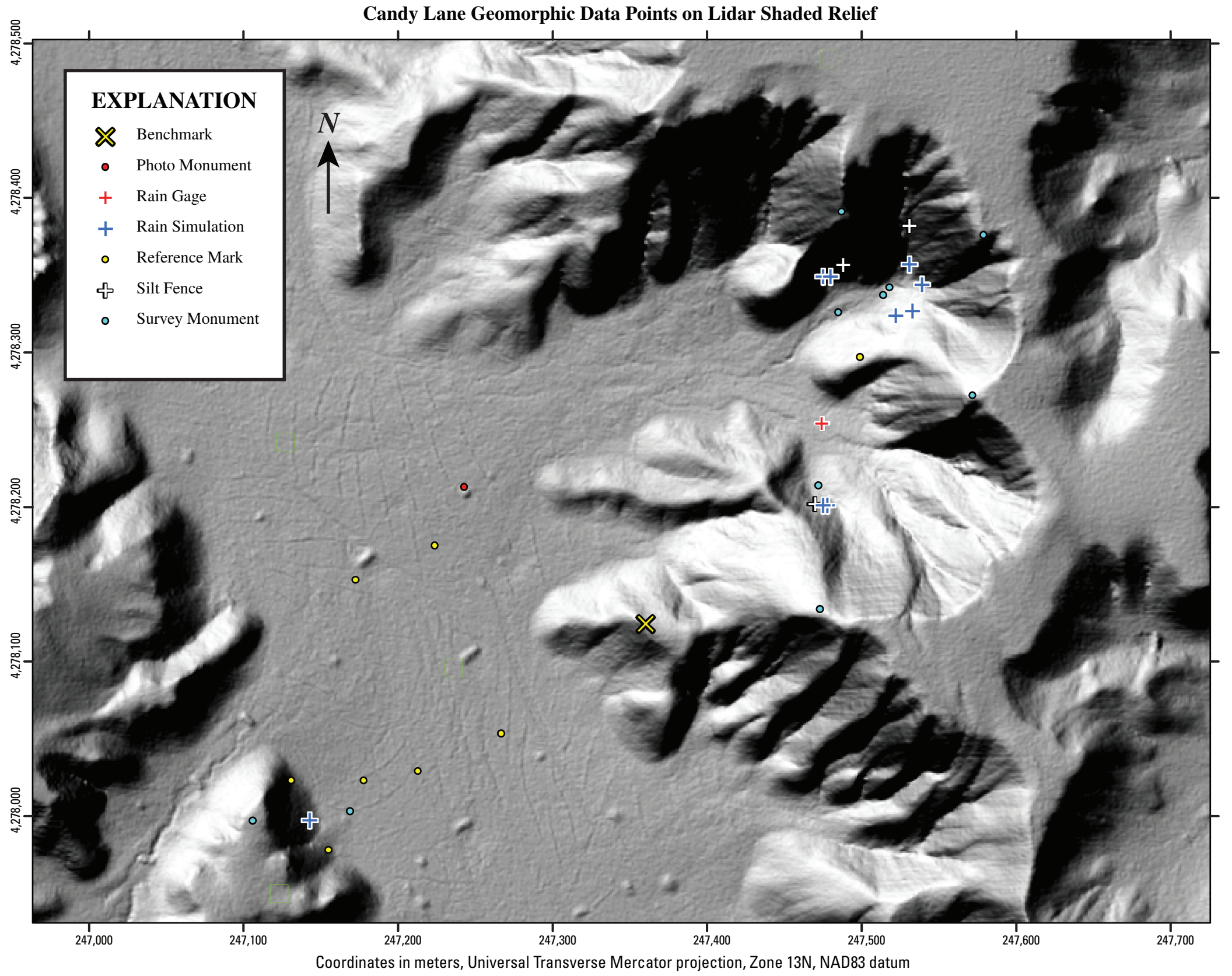

Figure 3. LIDAR image showing location of sampling sites in the Candy Lane study area. 
valley floors and less-steep hillslopes. Consequently, hillslopes with gradients between approximately 20 to 38 degrees occurring over a range of aspects (direction toward which hillslope faces) were selected for study (tables 1 and 2 at the end of this report).

All study sites initially were undisturbed or minimally disturbed by human or livestock activity. Rainfall-runoff simulations were performed on paired plots to test the effect of land-use change from undisturbed (control plot) to OHV disturbed (treatment plot). The control plot was systematically disturbed by a BLM employee on an off-road motorcycle.

\section{Topographic Surveys}

Rainfall-runoff simulations, small-plot microtopography, hillslope creep, and large-plot hillslope erosion measurements were made on hillslopes in GGNCA from 2003 to 2006. Topographic surveys from 2003 to 2005 determined geomorphic characteristics of the hillslopes (profile, gradient, aspect) on which these simulations and measurements were made (table 1). Because hillslope profile, gradient, and aspect can be extracted from digital elevation models for a larger geographic area using geographic information system (GIS) techniques, site characteristics, erosion processes, and other variables dependent on these geomorphic characteristics and quantified in this study can be extrapolated to other, non-instrumented locations.

Permanent bench marks (aluminum caps set in concrete) and survey reference marks (steel concrete-form stakes) were installed at the upper Elephant Skin Wash and Candy Lane study areas to facilitate relocation and replicate measurements at the study sites. Bench-mark and reference-mark coordinates originally were determined with a variety of hand-held GPS receivers and recorded in, or converted to, the Universal Transverse Mercator (UTM) projection, Zone $13 \mathrm{~N}$ coordinates, horizontal datum North American Datum of 1983 (NAD 83), and vertical datum North American Vertical Datum of 1988 (NAVD 88). Horizontal position error estimates for the GPS-determined coordinates ranged from plus or minus 4 to $12 \mathrm{~m}$. Consequently, reference-mark and other study-site monument coordinates are reported to a one-meter accuracy in tables 1 and 2, and are intended only to assist in relocating the study sites. All bench marks, reference marks, and other study-site monuments subsequently were surveyed to $0.003 \mathrm{~m}(0.01 \mathrm{ft})$ horizontal and vertical accuracy using a Topcon GTS-3B geodetic total station, a local, rectangular coordinate system, and an arbitrary, local vertical datum.

Personnel from the USGS National Geospatial Technical Operations Center II and Rocky Mountain Geographic Science Center conducted an onsite GPS survey in August and September 2005 to more precisely determine the coordinates and elevations of the two bench marks. These data were collected with dual frequency Ashtech Z-12 Global Positioning System receivers using static survey methods and, later, were postprocessed using Ashtech Office Suite (AOS) 2.0 software (J.J. Kosovich, U.S. Geological Survey, oral commun., September 2005). Bench-mark coordinates and elevations are presented to accuracies of 0.01 $\mathrm{m}$ in tables 1 and 2 .

Hillslope profile and hillslope gradient were determined by surveying a longitudinal profile from a high point at the top of the hillslope, down the relatively steep part, to the bottom of the hillslope where the gradient lessened abruptly using a Topcon GTS-3B geodetic total station. Topographic points down the hillslope were surveyed at approximately 3-m spacing along the transects. Hillslope aspect was determined onsite using a hand-held Brunton compass adjusted for local magnetic declination published on the most recent USGS 1:24,000-scale topographic map.

Cross sections and a longitudinal profile also were surveyed for one arroyo in the valley floor of the Candy Lane study area to estimate the headcut migration rate for this type of erosion (fig. 4). The arroyo cross sections were located to quantify the channel geometry of an unincised, a recently incised, and an older incised segment of the drainage system.

\section{Rainfall-Runoff Simulation}

Twenty-one small-plot, rainfall-runoff simulations were performed to characterize infiltration, runoff, and erosion on the study hillslopes. Simulations were performed on paired $1-\mathrm{m}^{2}$ plots to test the effect of land-use change from undisturbed (control plot) to OHV disturbed (treatment plot). The control plot was undisturbed with naturally occurring soil-crust structure and vegetation, whereas the paired treatment plot was carefully disturbed by a BLM motorcyclist making 12 passes through the plot. Simulated rainfall was designed to approximate the 100-year, 1-hour rainfall rate (38 mm, or $1.51 \mathrm{in}$.) for the Montrose, Colorado, region (Miller and others, 1973).

The simulated rainfall-delivery device was a modified design that was based primarily on the designs of previously used portable, small-plot devices (Wilcox and others, 1986; Byars and others, 1996; Benavidez-Solorio and MacDonald, 2001). A rectangular scaffold (Byars and others, 1996) with adjustable legs suitable for installation on variable-gradient hillslopes was constructed from 19-mm (3/4 in.) polyvinyl chloride (PVC) pipe and telescoping tent poles and set up onsite. The scaffold was enclosed with plastic tarps before the simulation began to reduce wind disturbance (fig. 5).

Simulated rainfall was delivered through a single Spraying Systems "Full Jet" FL-15 VS nozzle suspended approximately $2.0 \mathrm{~m}$ above the center of the $1-\mathrm{m}^{2}$ plot (Wilcox and others, 1986, fig. 1). The nozzle delivered droplets in a full-cone pattern 


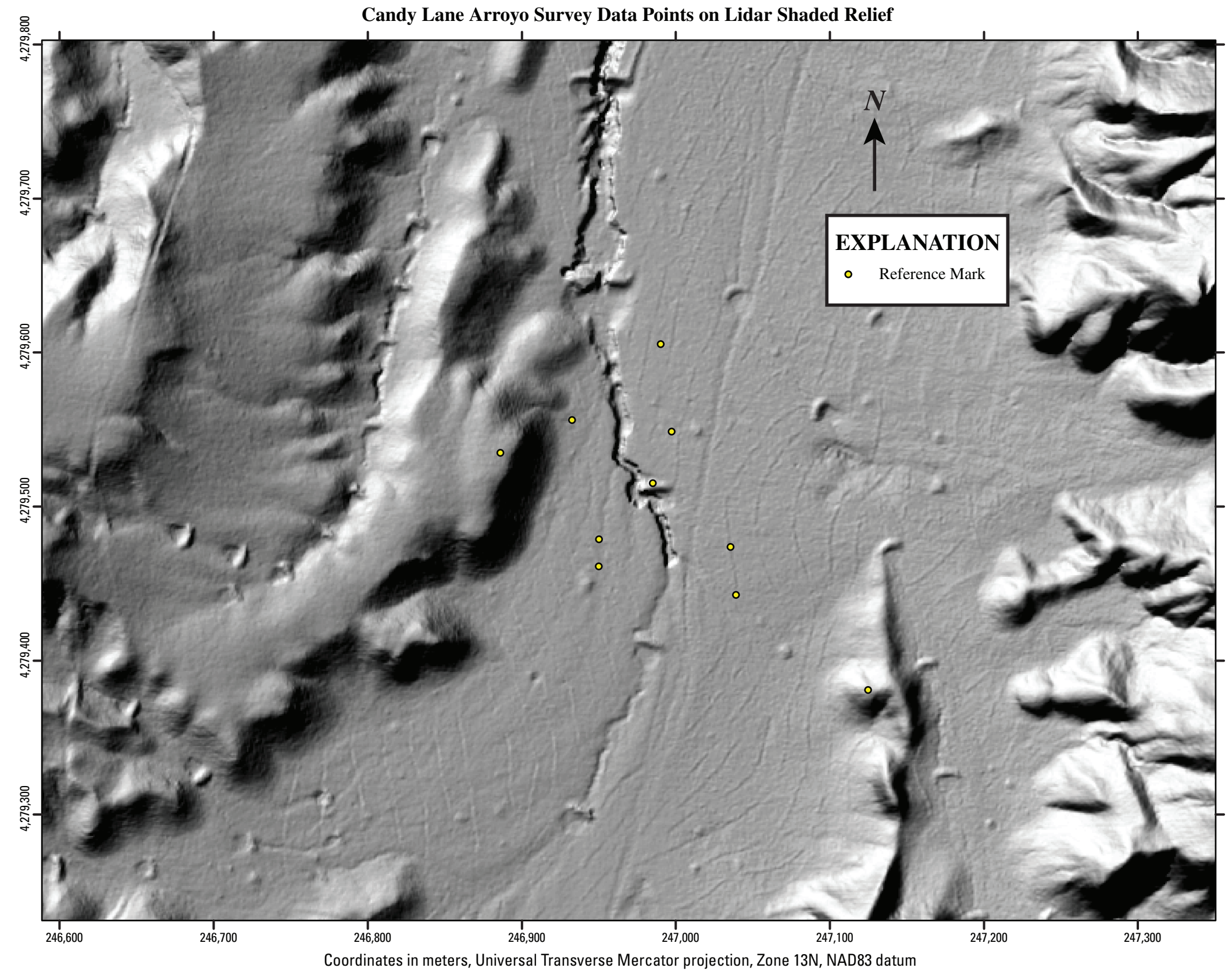

Figure 4. LIDAR image showing location of arroyo headcut in the Candy Lane study area. 


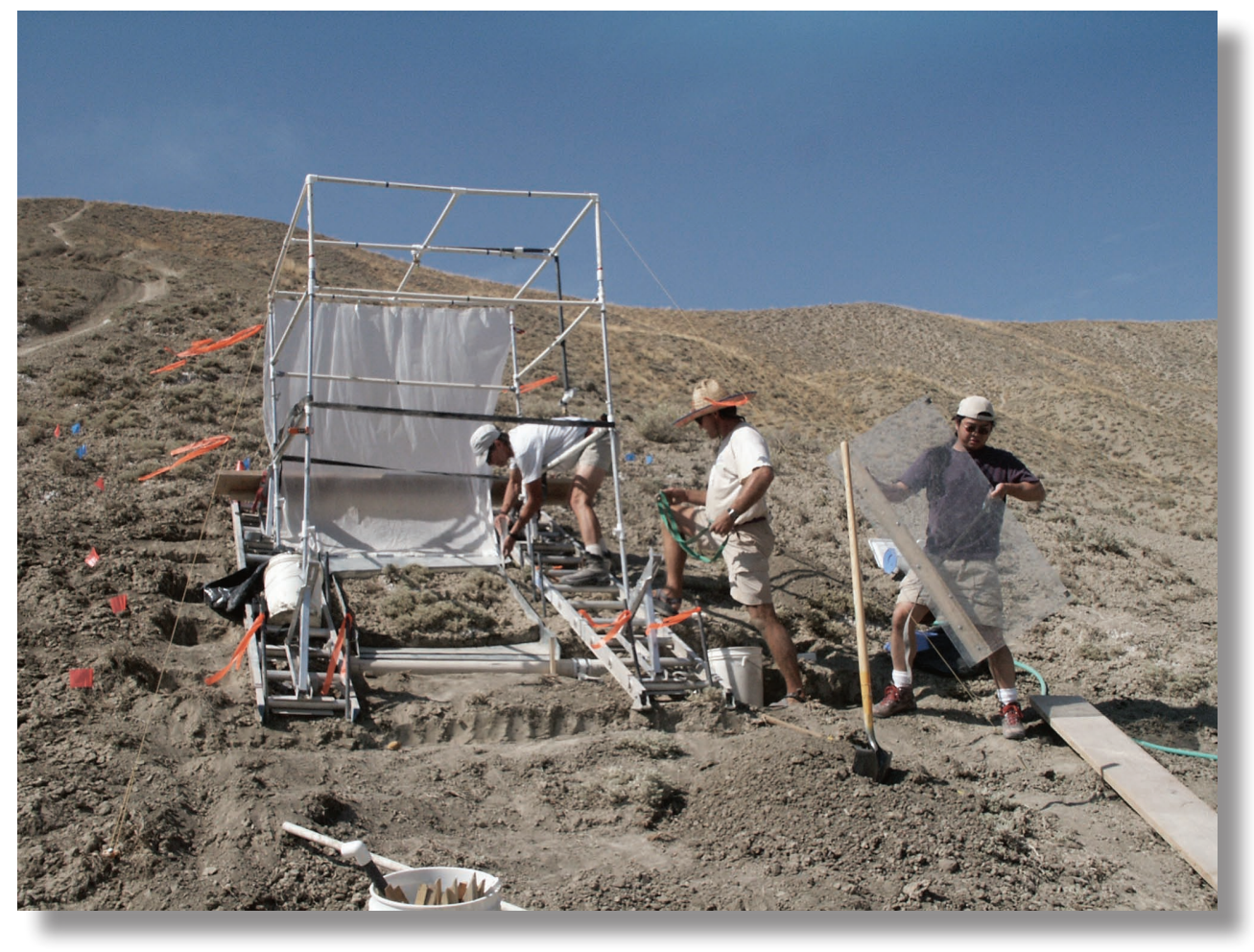

Figure 5. Simulated rainfallrunoff device set up on 1-square meter plot.

with a 120-degree angle. The nozzle was rated to deliver $1.5 \mathrm{gal} / \mathrm{min}$, with a droplet size of approximately $0.94-\mathrm{mm}$ diameter with $40 \mathrm{lb} / \mathrm{in}^{2}$ water pressure.

Water used in the simulations was withdrawn from approximately $200 \mathrm{~m}$ downstream from the discharge point of the Gunnison Tunnel into the South Canal, approximately $9 \mathrm{~km}$ east of Montrose, Colorado (fig. 1). This water was transported by truck to the study sites in a 200-gal polyethylene tank and applied to the study plot under pressure from a Wayne Water System 1/2 HP impeller-type water pump powered by electricity from a gasoline generator. Pressure was regulated manually with a valve at a gage on the scaffold and was kept between 38 and $40 \mathrm{lb} / \mathrm{in}^{2}$ throughout the simulation.

The 1- $\mathrm{m}^{2}$ rainfall-runoff simulation plot was chosen to be representative of the overall conditions of the larger study hillslope. Most $1-\mathrm{m}^{2}$ plots were located on a lower hillslope position, upslope from the hillslope foot or toe, due to water-supply proximity and upslope pumping limitations. A 3-m long section of 100-mm wide galvanized garden edging, folded at two corners formed three sides of the $1-\mathrm{m}^{2}$ plot border frame and was carefully tapped into the soil about $50 \mathrm{~mm}$. The fourth and downhill side of the plot was left unbordered where a 250-mm wide section of galvanized flashing was fitted between the frame sides to collect surface runoff from the plot. A slotted 100-mm diameter PVC pipe, placed horizontally across the hillslope and slightly sloping to one side or the other, received runoff from the flashing and directed it to a collection bucket adjacent to the scaffold. The collection apparatus was identical to the device used by Moody and Martin (2001, fig. 4.1) for their hillsloperunoff measurements.

Most rainfall simulations lasted for 90 minutes, a time sufficient to generate runoff from the test plots. Runoff and entrained sediment were collected in 500-mL bottles for size analysis, concentration measurement, hydrograph computation, and chemical analysis. When the runoff rate was relatively slow or the discharge low, the entire runoff was collected in several bottles for analysis. At higher rates and discharges, only part of the runoff was collected for analysis, but all runoff volume was measured in the field for total runoff computation.

\section{Runoff and Sediment Analysis}

Runoff from the rainfall simulations was collected to compute instantaneous discharge, total runoff hydrograph, suspendedsediment concentration, percentage of silt-and clay content, and chemical characteristics (J.R. Herring, U.S. Geological Survey, oral commun., 2003-2006). Soil crusts from within the 1-m² plots also were collected for particle-size analysis and Munsell color notation (Munsell Color, 1992) was recorded. 


\section{Runoff from Rainfall Simulations}

Runoff collected in bottles onsite from the rainfall simulations was analyzed at the USGS Colorado Water Science Center Sediment Laboratory in Lakewood, Colorado. Bottle tare weight (recorded before the simulation) was subtracted from the gross (filled) bottle weight to determine the net sample (water and entrained sediment) weight and total runoff volume. The total runoff volume for each bottle was divided by the bottle-collection time to determine an instantaneous discharge. Instantaneous discharges throughout the simulation were plotted against elapsed simulation time to create the total runoff (water plus entrained sediment) hydrograph (Frasier and others, 1998, fig. 1).

\section{Sediment Entrained in Runoff from Rainfall Simulations}

Supernatant water in the runoff samples was decanted after settling; the residual sediment was dried in an oven at $100^{\circ} \mathrm{C}$ for at least 24 hours and weighed to get the total sediment weight for each sample bottle (Guy, 1969). Sediment concentration in the runoff of each bottle was calculated as the dry weight of sediment divided by the residual of total runoff weight minus dry sediment weight. Instantaneous sediment concentrations were plotted against elapsed simulation time to create sediment concentration hydrographs, and the average sediment concentration for the entire simulation was computed. Total sediment yield for the simulation was the sum of all instantaneous sediment weights, plus an amount estimated for any unsampled runoff. The volume of onsite discarded runoff was known and was assumed to have a concentration equal to the simulation average concentration.

The residual sediment from three samples from each simulation were further analyzed to determine the size characteristics of sediment in the runoff from the rainfall simulation. These samples were deflocculated with a solution of sodium hexametaphosphate to aid in clay dispersal (Guy, 1969) and wet sieved through a $0.0625-\mathrm{mm}$ sieve to determine the percentages of sediment that were sand size or larger or finer than sand size (silt and clay). Samples from different times in each simulation (early-, middle-, and late-hydrograph periods) were analyzed to determine whether the sediment size characteristics changed as the simulation progressed. Because the total rainfall-simulation time differed from site to site, samples collected at approximately the 25th (early hydrograph), 50th (middle hydrograph), and 75th (late hydrograph) percentiles of the total, elapsed simulation time were analyzed.

\section{Sediment in Soil Crusts}

Dry soil crusts, approximately 10 to $30 \mathrm{~mm}$ thick, were collected onsite from the rainfall-runoff simulation plots and were analyzed for particle size and percentage of silt-and-clay content at the Bureau of Reclamation Soil Laboratory in Lakewood, Colorado. Grain-size data were obtained using a Coulter LS 100 Particle Size Analyzer, which is a laser-diffraction system capable of measuring particles between $0.0004 \mathrm{~mm}$ and $0.9 \mathrm{~mm}$ that are suspended in liquid. Particles may be any type of material including (but not limited to) sediment, soil, or biological samples. The particle-size analyzer is computer controlled and consists of a water-filled sample vessel with a built-in sonicator to disperse the particles, a laser-light source, lenses, and samplecell window. The suspended particles flow through the sample cell where the laser beam illuminates the dispersed sample. The particles scatter the light in patterns determined by their sizes. Arrays of photodetectors measure the intensity of the scattered light, and the particle sizes are determined by diffraction patterns using a Fourier lens. Volume calculations are automatically made from that data, but results may be customized to include volumes, surface-area distribution, statistical parameters, and comparative charts. Data may be presented as actual-size measurements or as sieve sizes.

The optimum amount of sample needed for particle-size measurement should be enough to provide a high signal-to-noise ratio but not enough to create problems with multiple scattering. One-half to $2 \mathrm{~g}$ of dry material are usually sufficient to provide an adequate sample. Larger samples may be characterized by analyzing subsamples and combining the results.

Dry samples were prepared for size analysis by physically disaggregating the soil crusts by hand as much as possible without causing grain-size reduction. The disaggregated material then was placed in a slurry consisting of sample, water, and sodium hexametaphosphate (to aid in clay dispersal), initially well mixed, stirred occasionally thereafter, and left for a minimum of 24 hours. Individual samples were poured through a \#35 sieve $(0.5 \mathrm{~mm})$ to remove larger particles such as fossil and gypsum fragments to avoid scratching the lenses; however, very little material was removed from any sample by this process.

\section{Microtopographic Measurement}

The soil-surface elevation within the 1- $\mathrm{m}^{2}$ rainfall-runoff simulation plots, referred to as the "microtopography," was measured in detail before and immediately after each rainfall simulation. Microtopographic measurements were made using a portable point-gage device, termed an "erosion bridge" by Moody and Martin (2001, fig. 4.4). The erosion bridge consisted of a 940-mm (37-in.) long, 25x51-mm (1x2-in.) aluminum channel bar with 5-mm (3/16-in.) holes spaced 10-mm (3/8-in.) apart, 
attached to a 1,220-mm (48-in.) long, 24x6-mm (1x1/4-in.) aluminum flat bar. The erosion bridge was leveled and clamped at a known reference elevation on permanent 16-mm (5/8-in.) steel concrete-form stakes driven into the ground on either side of the $1-\mathrm{m}^{2}$ plot border frame. Measurement of the soil-surface microtopography was made normal to the downslope direction by carefully lowering a 610-mm (24-in.) long, 5-mm (3/16-in.) diameter stainless-steel rod through every other hole in the erosion bridge until it contacted the soil surface (fig. 6). The length of the rod protruding above the top of the leveled erosion bridge was recorded to the nearest $1 \mathrm{~mm}$ and subtracted from the length of the rod; this remainder was subtracted from the elevation of the erosion bar to determine the soil-surface elevation. Forty-nine points were measured along the erosion bridge and within the $1-\mathrm{m}^{2}$ plot to determine a soil-surface profile. The difference in elevations at these discrete points before and after a rainfall simulation was computed, and the net change in microtopography along one-to-three normal-to-slope transects was calculated.

\section{Hillslope-Creep Measurement}

Hillslope creep in the upper soil horizons was measured over intervals of several months using 530-mm (20 3/4-in.) long wire flags inserted in the soil uphill from the rainfall-runoff simulation plots (figs. 2 and 3). The method used in this study was a modification of the method used by Godfrey (1997) on Mancos Shale hillslopes in Utah. Six wire flags were installed in a row across the hillslope at a known distance uphill from an aluminum bar attached to the permanent steel concrete-form stakes used in the microtopographic measurements. The uphill distance from the reference stakes ranged from 1.0 to $3.0 \mathrm{~m}$, depending on local soil and vegetation conditions at the time of installation. Uphill distance and the exposed length of the wire flags above the soil surface were recorded. Replicate measurements were made with a metal tape measure from a 1,220-mm (48-in.) long, 25x25-mm (1x1-in.) aluminum angle bar leveled and clamped to the reference stakes to the base of the exposed wire flag. Hillslope creep was calculated as the net change in position of the wire flags over the time interval between measurements (several months) and was reported in units of millimeters per year.

\section{Hillslope-Erosion Measurement}

Long-term (several months duration) hillslope erosion on study plots larger than the 1- $\mathrm{m}^{2}$ rainfall-runoff plots was measured at six locations adjacent to the rainfall-runoff simulation sites. These hillslope-erosion plots were located in the upper Elephant Skin Wash area on east, southeast, and northwest hillslope aspects (fig. 2) and in the Candy Lane area on south,

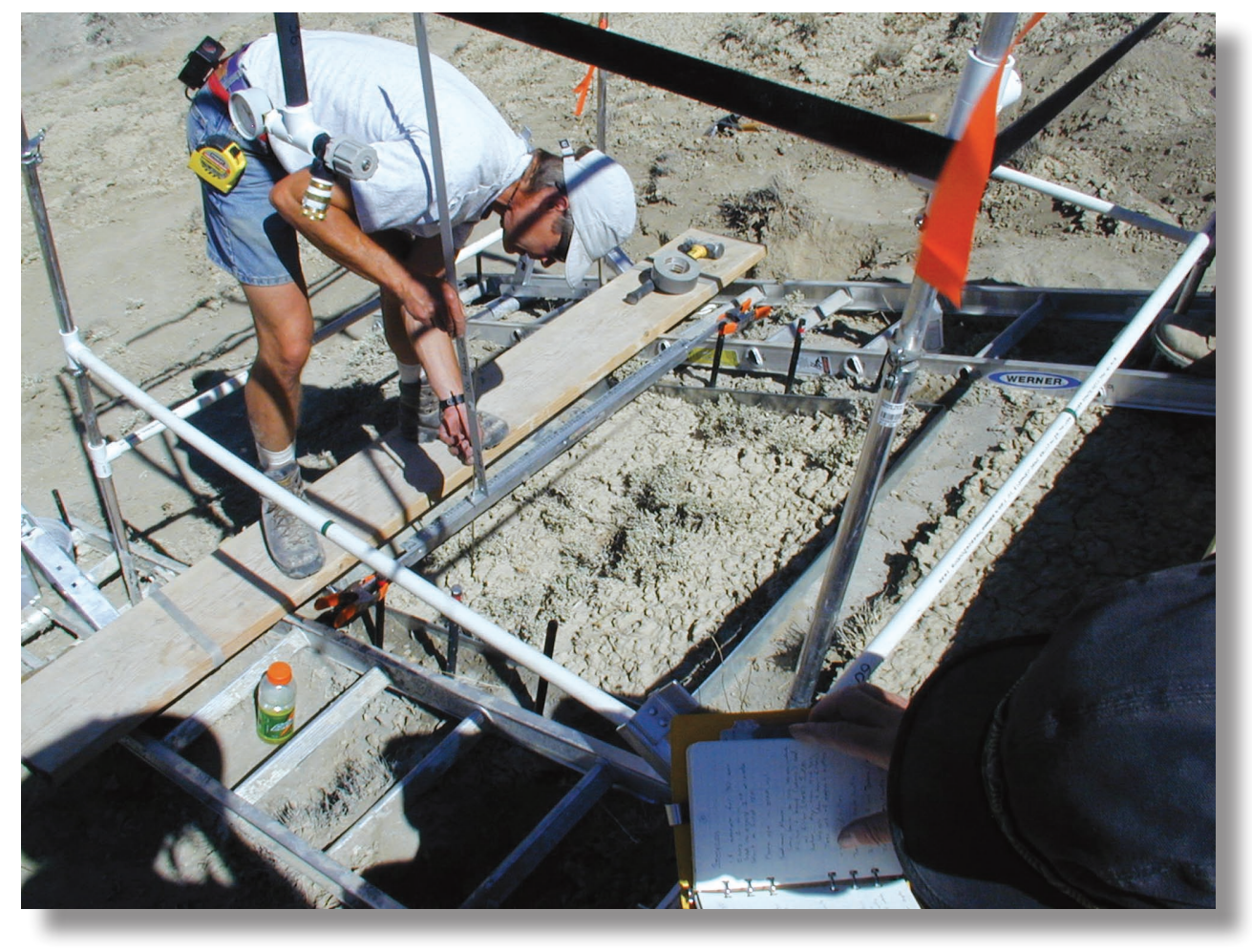

Figure 6. Erosion bridge and microtopographic measurement. 
west, and north hillslope aspects (fig. 3). The rectangular plots ranged in size from $4 \times 7 \mathrm{~m}\left(28 \mathrm{~m}^{2}\right)$ to $\left.5 \times 10 \mathrm{~m}^{(50} \mathrm{m}^{2}\right)$ with the long dimension oriented downslope. Each plot had an impervious plastic barrier on the uphill, cross-slope boundary to prevent upslope runoff from entering the study plot. A 610-mm (24-in.) high, woven silt fence was installed at the downhill plot boundary to collect sediment and pass water derived from within the plot boundaries (fig. 7). The silt-fence fabric was 915-mm (36-in.) wide, and a 305-mm (12-in.) wide portion of the fabric was anchored and buried to prevent sediment and runoff from passing beneath the fence (Robichaud and Brown, 2002, fig. 2). The mesh opening size of the commercial-grade silt-fence fabric was not determined, but onsite examination indicated most of the sediment from the plot was trapped by the fabric.

Sediment trapped behind the silt fence was removed periodically, weighed onsite ("field weight"), air dried in the sediment laboratory, and reweighed to determine the sediment yield (megagrams per hectare) and seasonal erosion rate (megagrams per hectare per year). If the collected sample was large and moist, an aggregate subsample of captured sediment was weighed onsite, dried, and reweighed in the laboratory to determine a moisture correction factor that subsequently was applied to the field sample to calculate the dry sediment weight of the entire collected sample. After the initial silt-fence installation and after periodic removal of accumulated sediment, red carpenter's chalk was dusted on the ground surface to create a stratigraphic marker visible beneath sediment accumulated in the succeeding runoff period.

\section{Precipitation Measurement}

Precipitation was measured in the upper Elephant Skin Wash and Candy Lane study areas using Belfort 5-780 series universal recording rain gages from October 2004 through April 2006. A single gage was located at each study area in a location with minimal topographic or vegetation interference (figs. 2 and 3). These weighing-type gages recorded rain and snow precipitation. Recording charts, showing individual precipitation events as well as the accumulated precipitation as a depth, were retrieved and replaced approximately every 3 to 5 months. The precipitation gage data provided a means to determine the magnitude and duration of individual storms as well as the total precipitation during intervals between the hillslope-creep and hillslope-erosion (silt-fence) measurements.

Precipitation and climate data also are routinely collected at the Montrose \#2 climate station by the National Oceanic and Atmospheric Administration's National Climate Data Center (NCDC). Precipitation data from the Montrose \#2 climate station (National Oceanic and Atmospheric Administration, 2007) was compared to data from the upper Elephant Skin Wash precipitation gage (approximately $10 \mathrm{~km}$ northeast from the Montrose \#2 climate station) and Candy Lane precipitation gage (approximately $14 \mathrm{~km}$ north from the Montrose \#2 climate station) to determine site-to-site correlations and to provide study-site precipitation estimates for earlier periods (before October 2004) when hillslope-creep measurements were made.

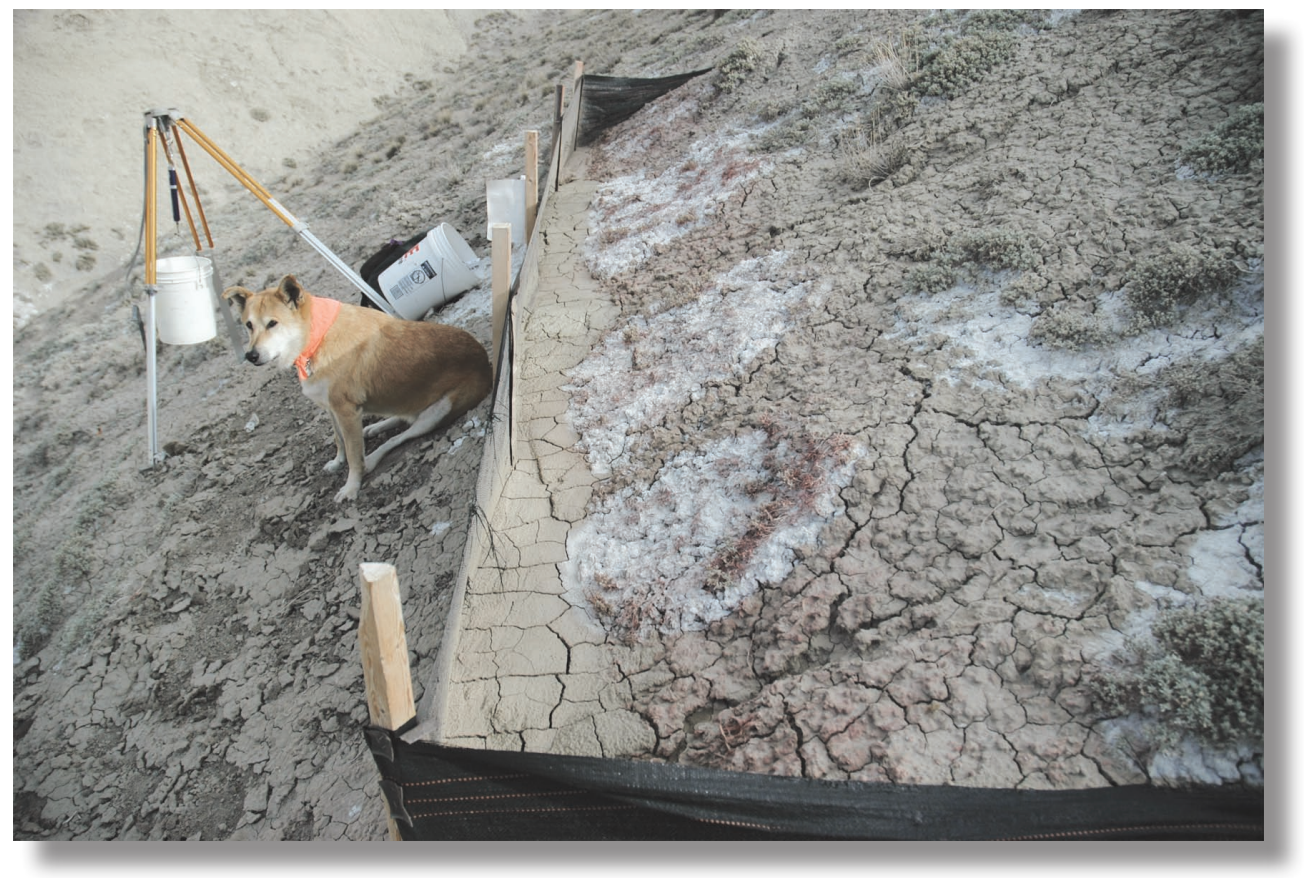

Figure 7. Silt fence at a hillslope-erosion measurement site. 


\section{Rainfall-Runoff and Erosion Data}

Rainfall-runoff, erosion, and additional data were collected from hillslope study sites in two areas of the Gunnison Gorge National Conservation Area (GGNCA) - the upper Elephant Skin Wash area and the Candy Lane area (fig. 1). Six topographic surveys were made on hillslopes in the upper Elephant Skin Wash area (fig. 2), and five were made in the Candy Lane area (fig. 3). Hillslope profiles were generated from the topographic surveys to determine the longitudinal properties (length, curvature, and so forth), aspect, and the average and local hillslope gradients.

Most hillslopes had a long, straight middle portion with relatively constant gradient, a geomorphic feature referred to as the "valley-side slope" by Hack and Goodlett (1960), or the "backslope" by Ruhe and Walker (1968). All hillslopes also has a convex upper portion ("nose" in the Hack and Goodlett nomenclature, or "shoulder" in the Ruhe and Walker nomenclature), and a concave lower portion ("footslope" in both the Hack and Goodlett and Ruhe and Walker nomenclatures) (fig. 8). Average hillslope gradients were calculated from just below the shoulder to just above the footslope and ranged from 20.2 to 37.9 degrees with a mean gradient of 28.5 degrees (table 3 at the end of this report).

Paired rainfall-runoff simulation plots, undisturbed and disturbed, were located on the lower area of the straight hillslope portion (fig. 8) and were positioned to represent the general geomorphic and vegetation characteristics of the larger hillslope area on which they were located. The gradients of the $1-\mathrm{m}^{2}$ rainfall-runoff simulation plots ranged from 21.8 to 37.5 degrees with a mean of 29.9 degrees on undisturbed (control) plots and from 23.0 to 35.0 degrees with a mean of 29.4 degrees on (treatment) plots disturbed by OHV tracks (table 3 ). Total vegetation cover in the $1-\mathrm{m}^{2}$ rainfall simulation plots was estimated visually before rainfall simulation and, in the case of the treatment plots intentionally disturbed by motorcycle tracks, after the controlled disturbance and before the rainfall simulation. Vegetation cover ranged from 0 to 25 percent for the undisturbed plots and from 0 to 10 percent for the disturbed plots (table 3).

Rainfall simulations were performed on $211-\mathrm{m}^{2}$ plots (one undisturbed plot, CS.24E_U, did not have a paired treatment). Three of the plots (sites ES.40E_U2, ES.40E_D2, and CP.8NW_D2) had a second rainfall simulation performed approximately 24 hours after the first simulation, while the soil was still wet, to simulate rainfall on successive days. Most rainfall simulations were 90 minutes in duration; however, three were shortened due to equipment malfunctions or soil liquefaction (plots ES.40NW_U, CP.8S_U, and CP.8S_D, table 3). Two plots (ES.9SW_D and ES.40NW_D) had 120-minute simulations because initial runoff from these plots did not occur until well into the simulation.

The target simulated-rainfall application rate was the 100-year, 1-hour storm at Montrose, Colorado, or $38 \mathrm{~mm}$; however, most simulations lasted for 90 minutes, and mean rainfall application rates ranged from 38.3 to $74.4 \mathrm{~mm} / \mathrm{h}$ due to uncontrollable variations in rainfall-simulator hydraulic performance at a constant water pressure (table 3). Total rainfall depth during the simulations ranged from 52.4 to $107.0 \mathrm{~mm}$ because of variation in simulation duration and simulator hydraulic performance. Consequently, all runoff, infiltration, and erosion data were normalized to hourly rates using the total applied rainfall to allow site-to-site comparisons.

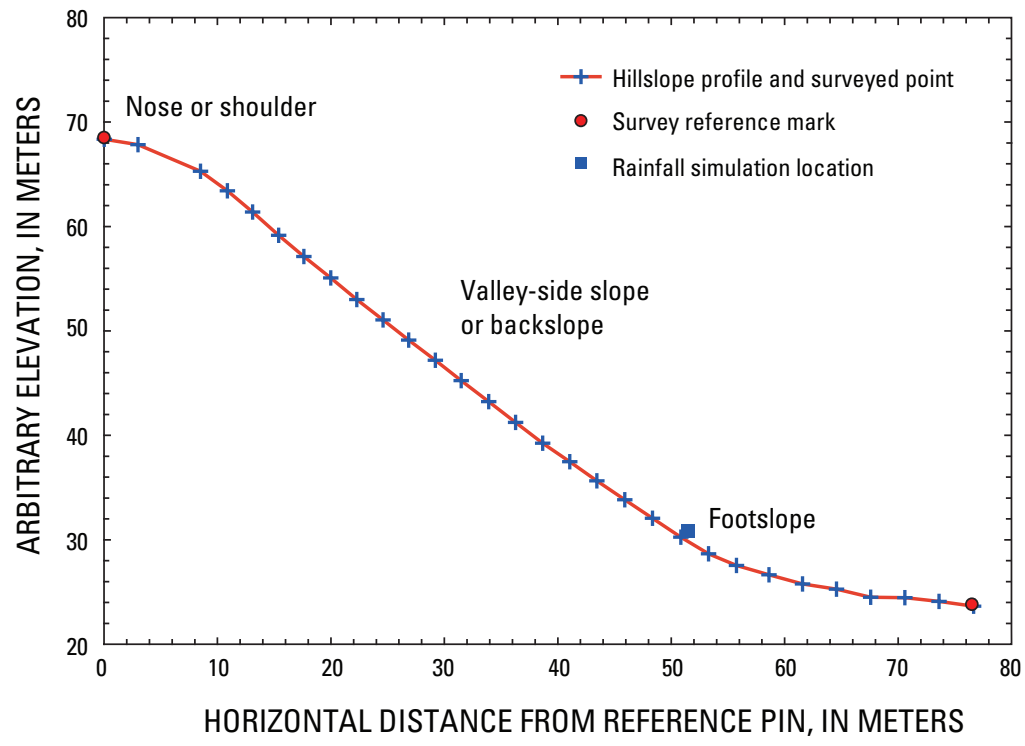

Figure 8. Surveyed hillslope profile and geomorphic terminology at CP.8W. 
Runoff and eroded sediment from the rainfall simulation plots were measured and normalized to hourly rates using the total applied rainfall. The normalized total runoff rate (water and sediment) ranged from 335 to $15,856 \mathrm{~mL} / \mathrm{m}^{2} / \mathrm{h}$, with a mean of $5,321 \mathrm{~mL} / \mathrm{m}^{2} / \mathrm{h}$ from undisturbed plots, and ranged from 366 to $17,929 \mathrm{~mL} / \mathrm{m}^{2} / \mathrm{h}$, with a mean of $5,340 \mathrm{~mL} / \mathrm{m}^{2} / \mathrm{h}$ from disturbed plots (table 3). Mean values were calculated only from results of the single-event simulations and the first day of multiple-day simulations; second-day data from the three multiple-day sites (ES.40E_U2, ES.40E_D2, and CP.8NW_D2) were excluded from mean calculations.

Runoff was collected periodically during the simulations, and instantaneous runoff rates were calculated from the sample volume (milliliters) and sample duration (minutes). Hydrographs of total runoff were derived from plots of instantaneous discharge (milliliters per minute) and elapsed simulation time to determine the runoff and infiltration characteristics of each $1-\mathrm{m}^{2}$ simulation plot (fig. 9).

The simulation infiltration volume was calculated as the difference between the total simulation rainfall applied to the $1-\mathrm{m}^{2}$ plot and total runoff from the plot, in milliliters (table 3). Infiltration rates were normalized to the applied rainfall rate and ranged from 22,144 to $37,665 \mathrm{~mL} / \mathrm{m}^{2} / \mathrm{h}$, with a mean of $32,679 \mathrm{~mL} / \mathrm{m}^{2} / \mathrm{h}$ from undisturbed (control) plots, and from 20,071 to 37,634 $\mathrm{mL} / \mathrm{m}^{2} / \mathrm{h}$, with a mean of $32,660 \mathrm{~mL} / \mathrm{m}^{2} / \mathrm{h}$ from disturbed (treatment) plots. Most $1-\mathrm{m}^{2}$ plots were excavated at one or two locations after the rainfall simulations to observe the mean wetting-front depth, the depth in the soil profile to which visible wetting during the rainfall simulation had occurred in the otherwise dry soil. Mean wetting front depths generally were greater on plots where normalized total runoff rates were less and ranged from 52.5 to $620.0 \mathrm{~mm}$, with a mean of $238.6 \mathrm{~mm}$ beneath undisturbed plots and from 70.0 to $330.0 \mathrm{~mm}$, with a mean of $189.7 \mathrm{~mm}$ beneath disturbed plots.

The mean sediment concentration in the runoff ranged from 0.32 to $55.46 \mathrm{~g} / \mathrm{L}$, with a mean of $23.86 \mathrm{~g} / \mathrm{L}$ for the undisturbed plots, and from 0.16 to $75.90 \mathrm{~g} / \mathrm{L}$, with a mean of $20.84 \mathrm{~g} / \mathrm{L}$ for the disturbed plots. Sediment-concentration hydrographs were derived from the instantaneous concentration and elapsed simulation time of each sample (fig. 10). Normalized sediment yield ranged from 0.10 to $816.83 \mathrm{~g} / \mathrm{m}^{2} / \mathrm{h}$, with a mean of $215.67 \mathrm{~g} / \mathrm{m}^{2} / \mathrm{h}$ for the undisturbed plots, and from 0.14 to $773.73 \mathrm{~g} / \mathrm{m}^{2} / \mathrm{h}$, with a mean of $217.03 \mathrm{~g} / \mathrm{m}^{2} / \mathrm{h}$ for the disturbed plots (table 3 ).

A sediment-size analysis was performed on selected samples collected at approximately the $25^{\text {th }}$ (early hydrograph), $50^{\text {th }}$ (mid-hydrograph), and $75^{\text {th }}$ (late hydrograph) percentiles of simulation elapsed time to determine the percentage of sand and percentage of silt and clay content in the runoff. For the undisturbed (control) plots, the mean percentage of silt and clay content in runoff was 91.1, 92.2, and 85.3 percent in the early, mid-, and late hydrograph periods, respectively, with a mean silt and clay content of 89.5 percent (table 3). For the disturbed (treatment) plots, the mean percentage of silt and clay content in runoff was 95.6, 94.4, and 94.9 percent in the early, mid-, and late hydrograph periods, respectively, with a mean silt and clay content of 94.7 percent.

Sediment-size analysis also was performed on the dry soil crust collected from the 1- $\mathrm{m}^{2}$ rainfall-runoff simulation plots. The percentage of silt and clay content of the undisturbed plots ranged from 72.8 to 94.3 percent, with a mean of 90.1 percent. The median particle size of soil-crust sediment from undisturbed plots ranged from 0.0035 to $0.0144 \mathrm{~mm}$, with a mean of 0.0065 (table 3). The percentage of silt and clay content of the disturbed plots ranged from 82.6 to 95.8 percent, with a mean of 91.0 percent (table 3). The median particle size of soil-crust sediment from disturbed plots ranged from 0.0050 to $0.0095 \mathrm{~mm}$, with a mean of $0.0065 \mathrm{~mm}$.

Soil-surface microtopographic measurements were made normal to the downslope direction on each 1- $\mathrm{m}^{2}$ rainfall-runoff simulation plot before and after each simulation (fig. 11). The elevation difference at 49 discrete points before and after a rainfall simulation was computed along one-to-three transects, and the net change in microtopography along the plot transects was calculated (table 4 at the end of this report).

Negative elevation changes at a point were caused by soil detachment, micro-rill formation, compaction of loose soil particles (ravel), and slumping or solifluction. Positive elevation changes at a point were caused by soil accumulation from upslope erosion, swelling, and slumping or solifluction. Most rainfall-runoff plots showed a combination of elevation increases and decreases at discrete measurement points indicating that multiple processes were active. Net microtopographic elevation changes ranged from -6 to $+10 \mathrm{~mm}$ in all but one undisturbed (control) plots and from -7 to $+8 \mathrm{~mm}$ in all but one disturbed (treatment) plots (table 4). One undisturbed plot (CP.8W_U) had a $-32 \mathrm{~mm}$ net elevation change due to a rapid, downslope, solifluction displacement at the end of the 90-minute rainfall simulation. One disturbed plot (CP.8NW_D2) had a $-13 \mathrm{~mm}$ net elevation change on the second day of a 2-day experiment after showing no net elevation change on the first day (measurement CP.8NW_D1).

Hillslope creep in the upper soil horizons was measured over intervals of several months at locations 1 to $3 \mathrm{~m}$ upslope from each rainfall-runoff simulation site. Seasonal net hillslope-creep rates were calculated as the average net change in position of six wire flags over the time interval between measurements and were reported in units of millimeters per day and millimeters per year (table 5 at the end of this report). Annual net hillslope-creep rates also were calculated for two periods, April or May 2004 to April 2005, and April 2005 to April 2006. Because the wire flag positions were measured from a reference bar to their insertion point in the soil, the precision of creep measurements was affected by changes in soil-surface irregularities and vegetation growth that may have interfered with a straight-line, metal tape measurement from the reference bar. 


\section{(A) ES.9SE}

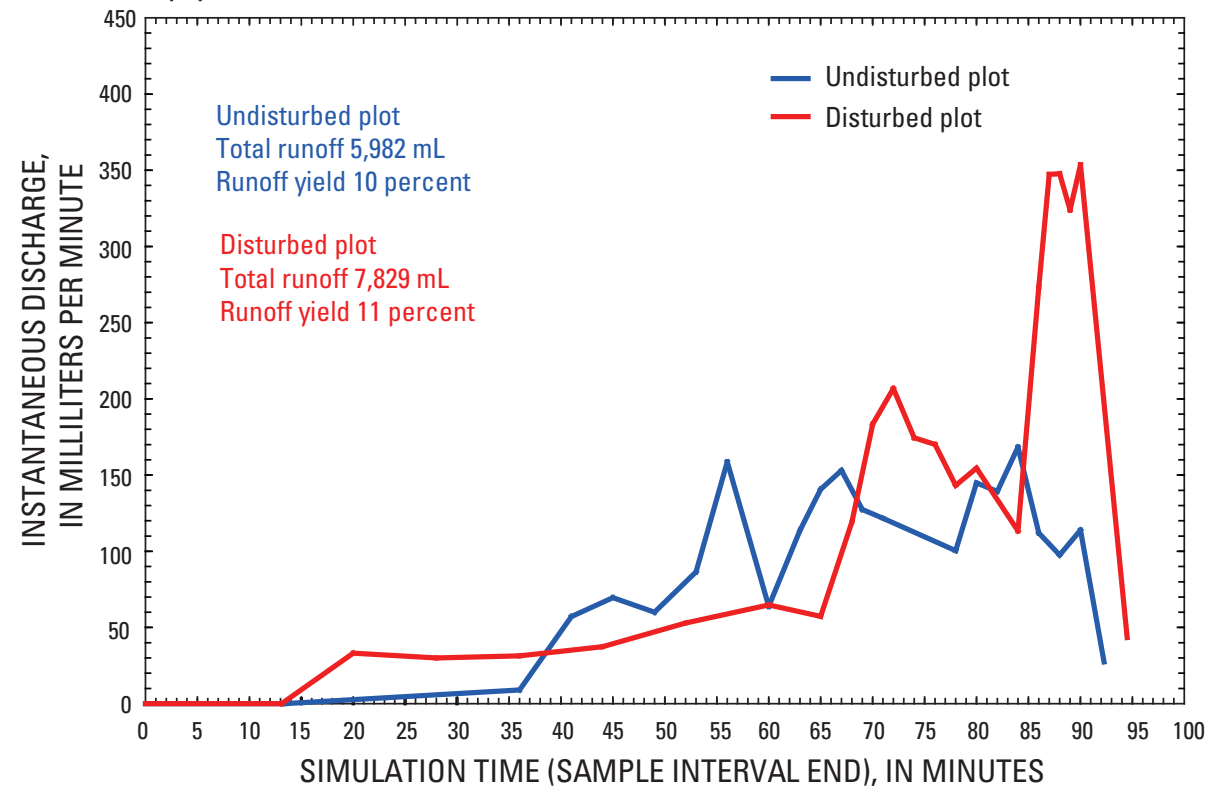

(B) ES.9NW

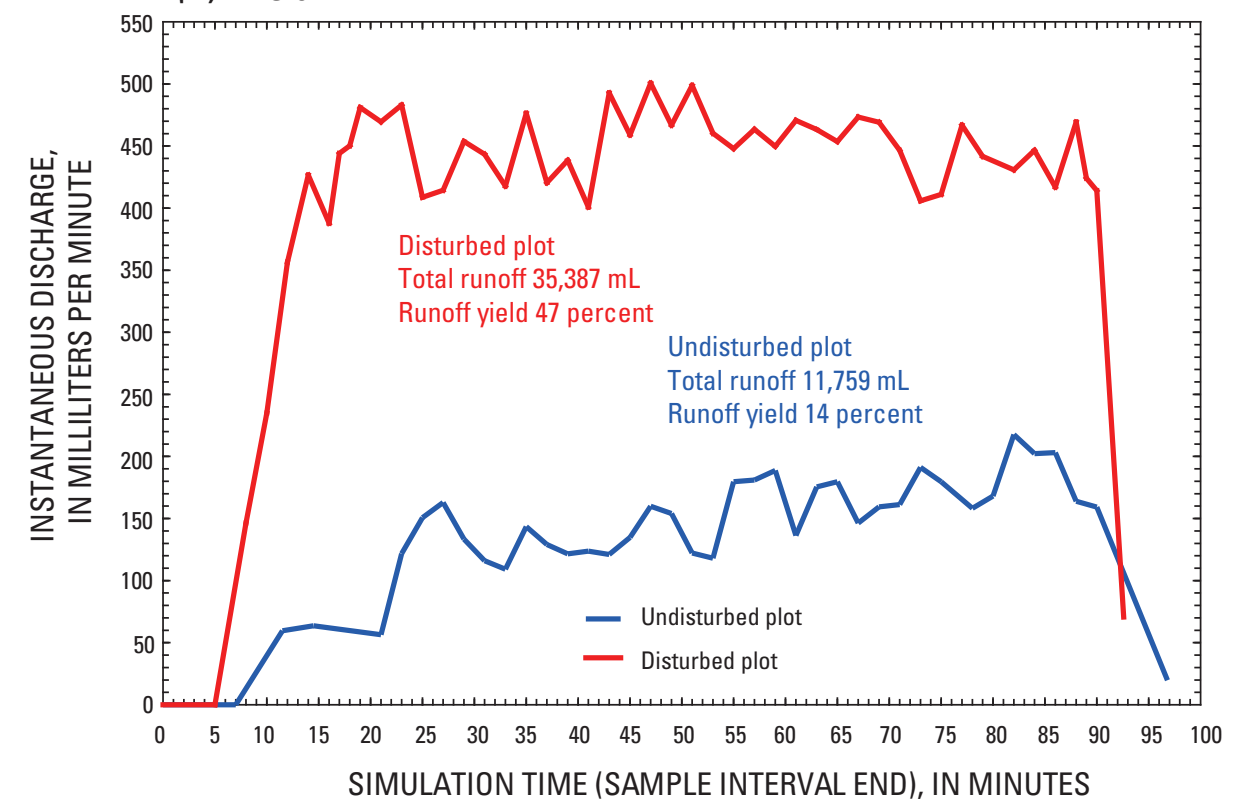

Figure 9. Total-runoff hydrographs from rainfall simulations. 

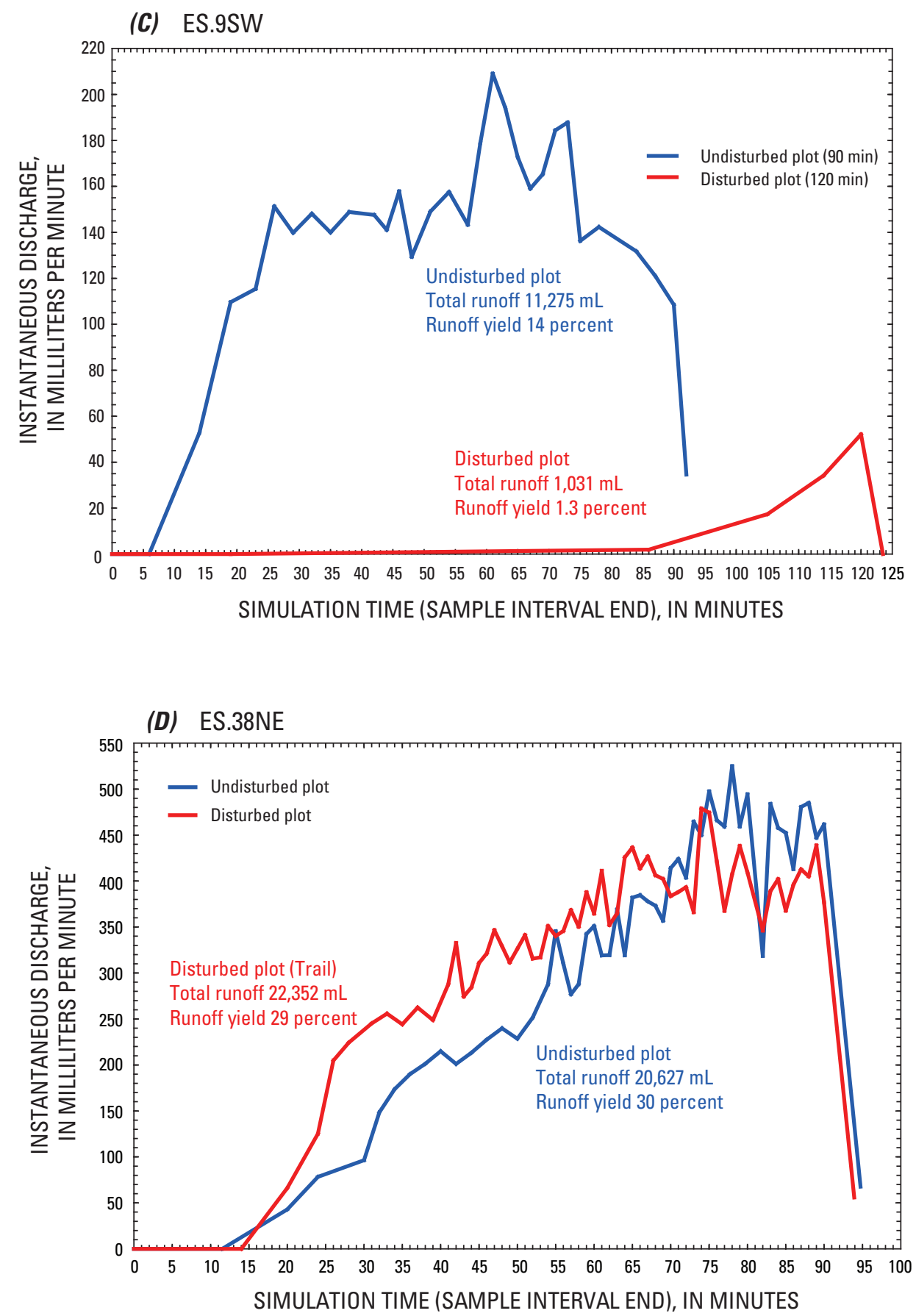

Figure 9. Total-runoff hydrographs from rainfall simulations. - Continued. 


\section{(E) ES.40E}
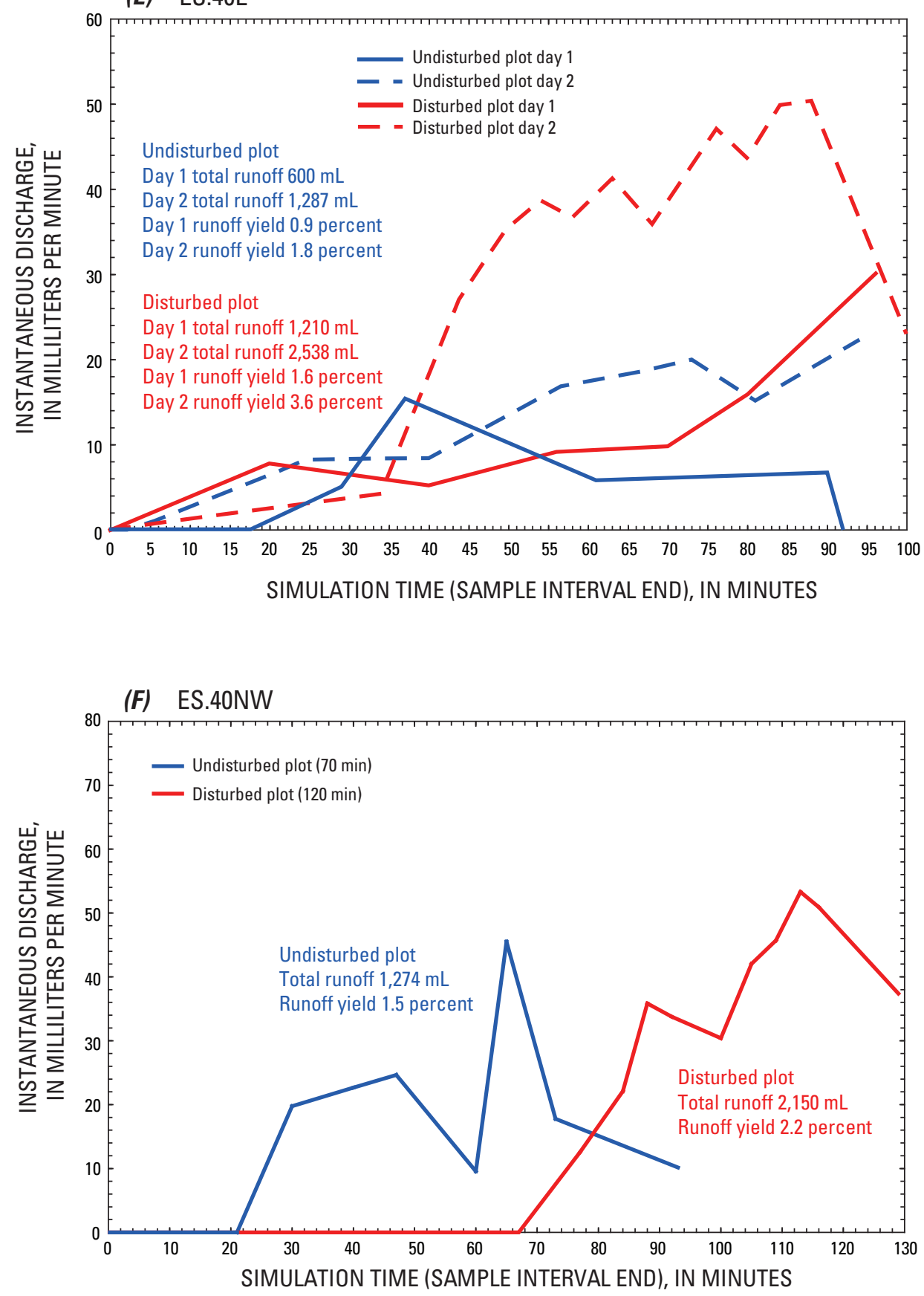

Figure 9. Total-runoff hydrographs from rainfall simulations.-Continued. 
(G) CP.8S

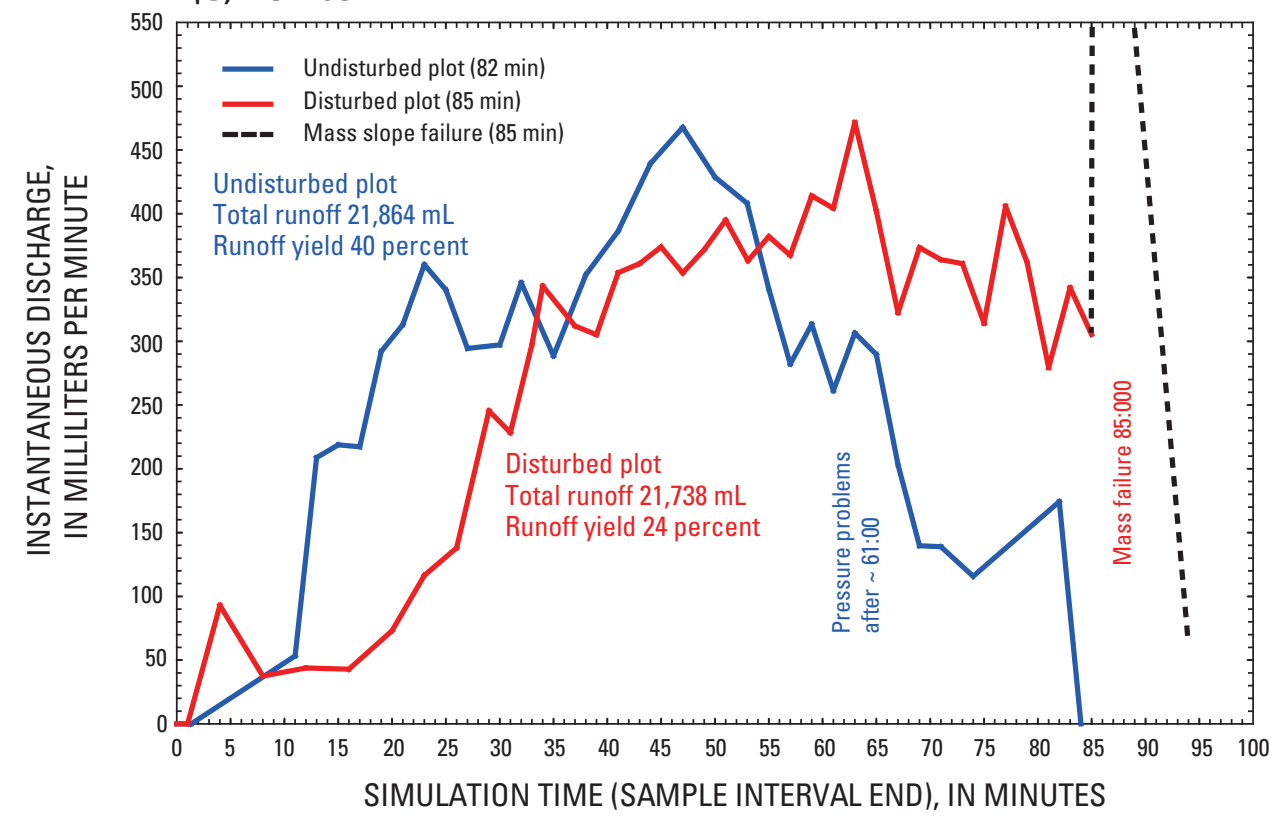

(H) $\mathrm{CP} .8 \mathrm{~W}$

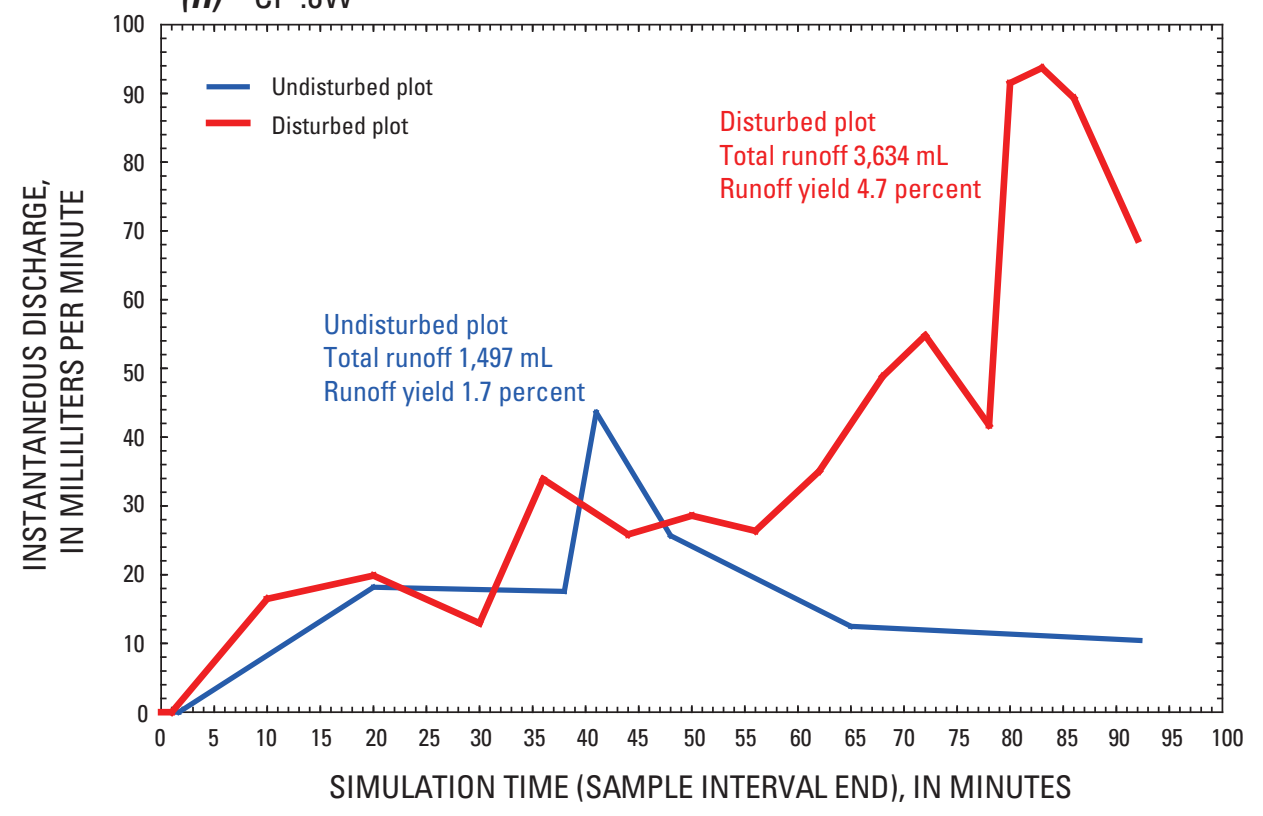

Figure 9. Total-runoff hydrographs from rainfall simulations. - Continued. 

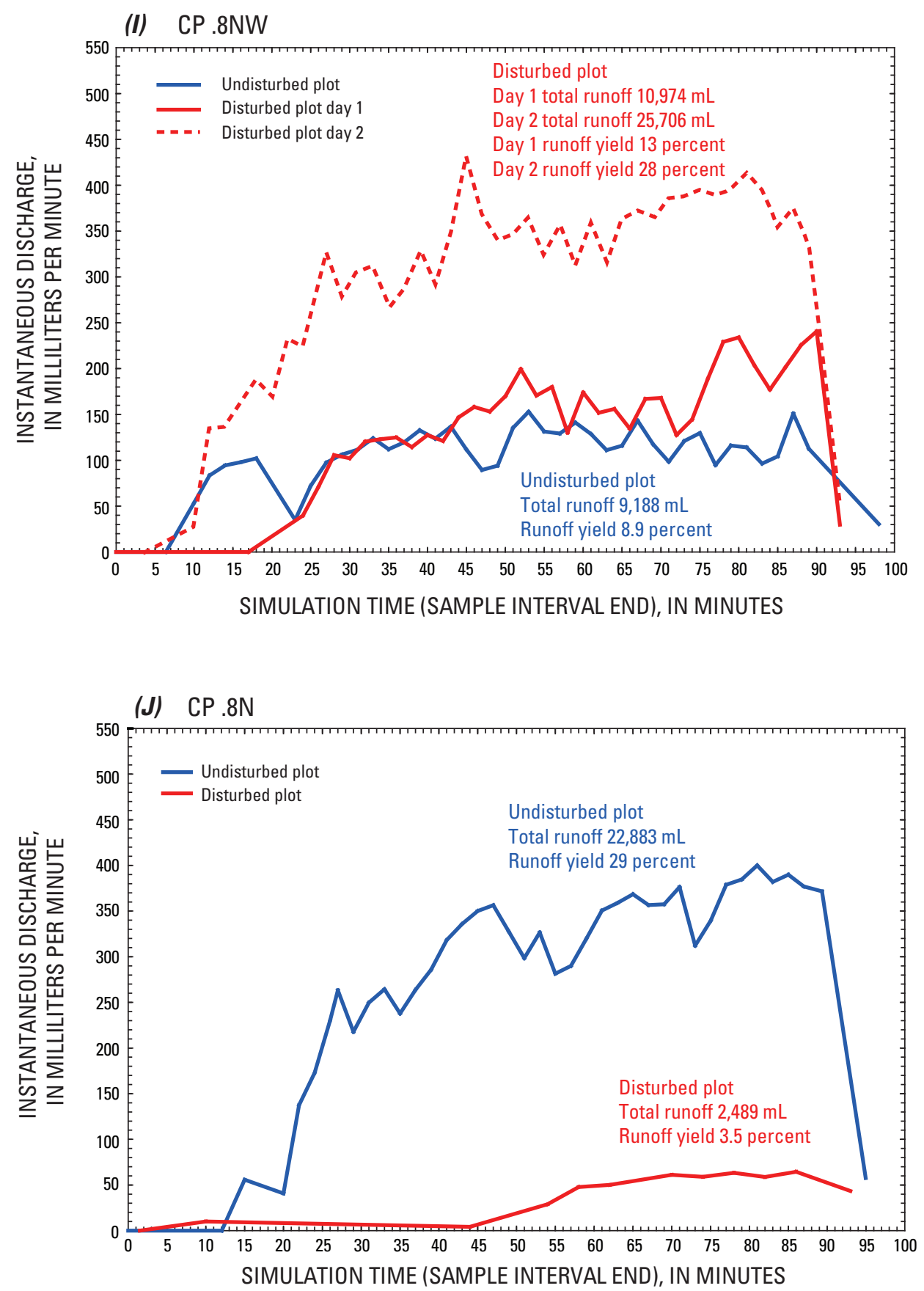

Figure 9. Total-runoff hydrographs from rainfall simulations.-Continued. 
(A) ES9.SE

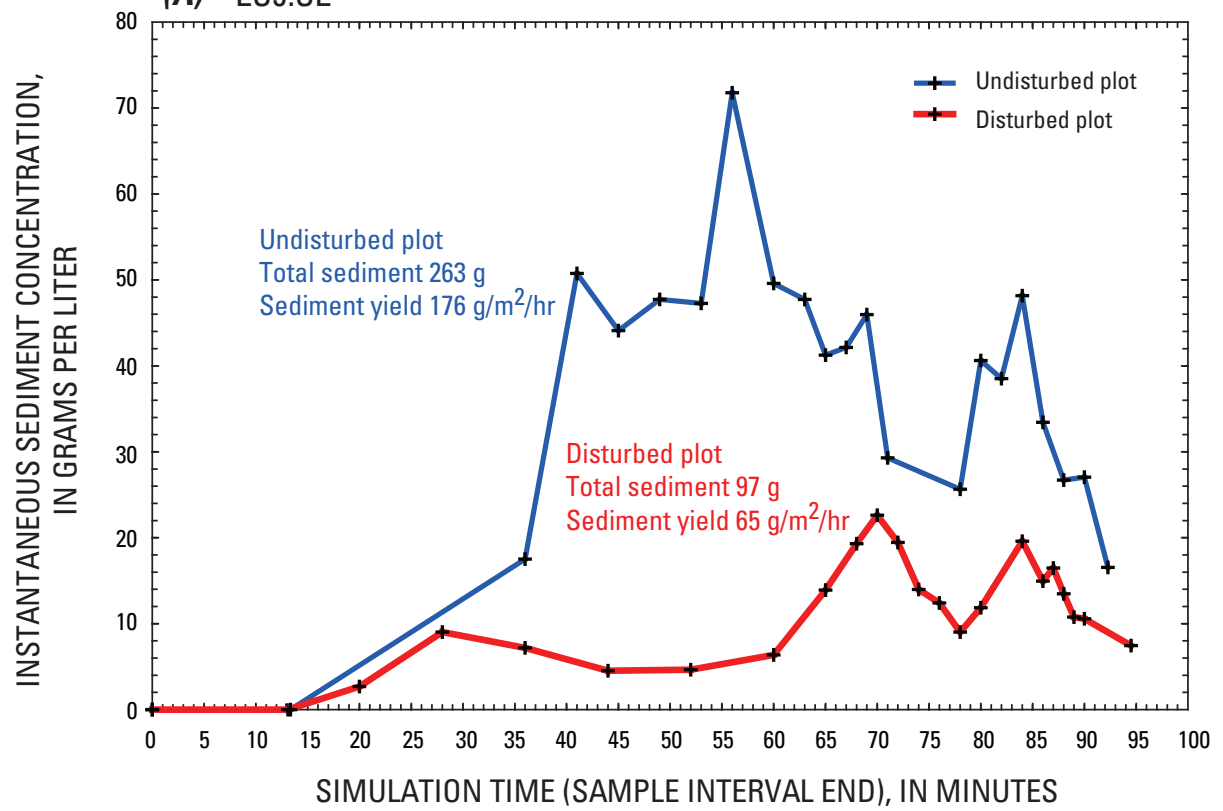

(B) ES9.NW

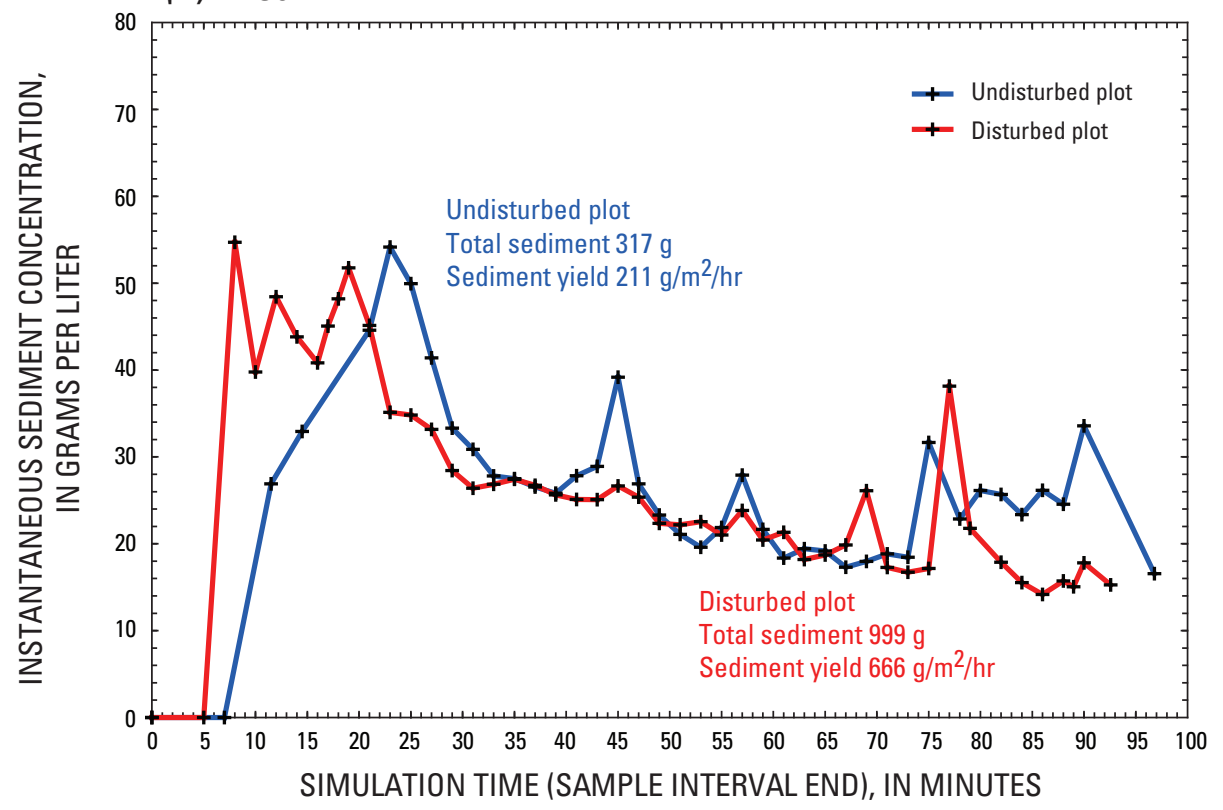

Figure 10. Sediment-concentration hydrographs from rainfall simulations. 
(C) ES.9SW

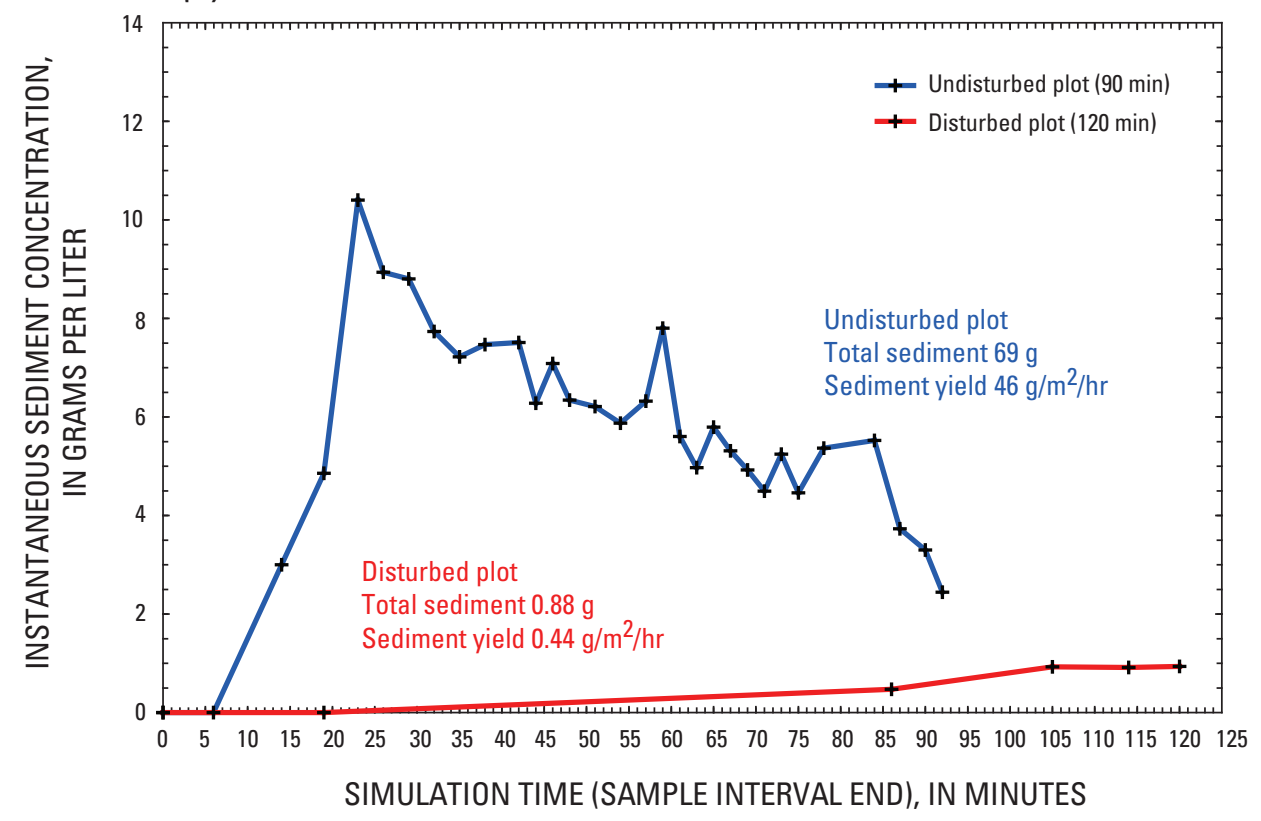

(D) ES38.NE

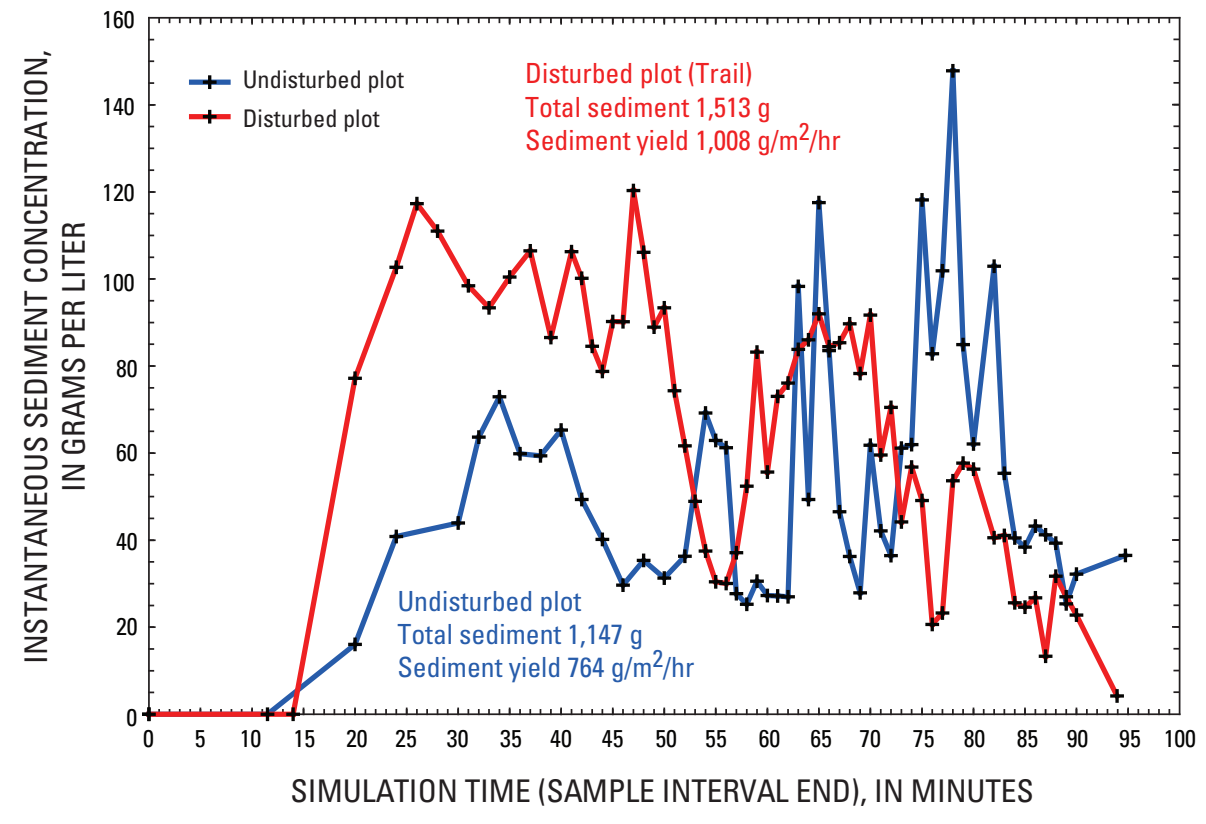

Figure 10. Sediment-concentration hydrographs from rainfall simulations.-Continued 


\section{(E) ES.40E}

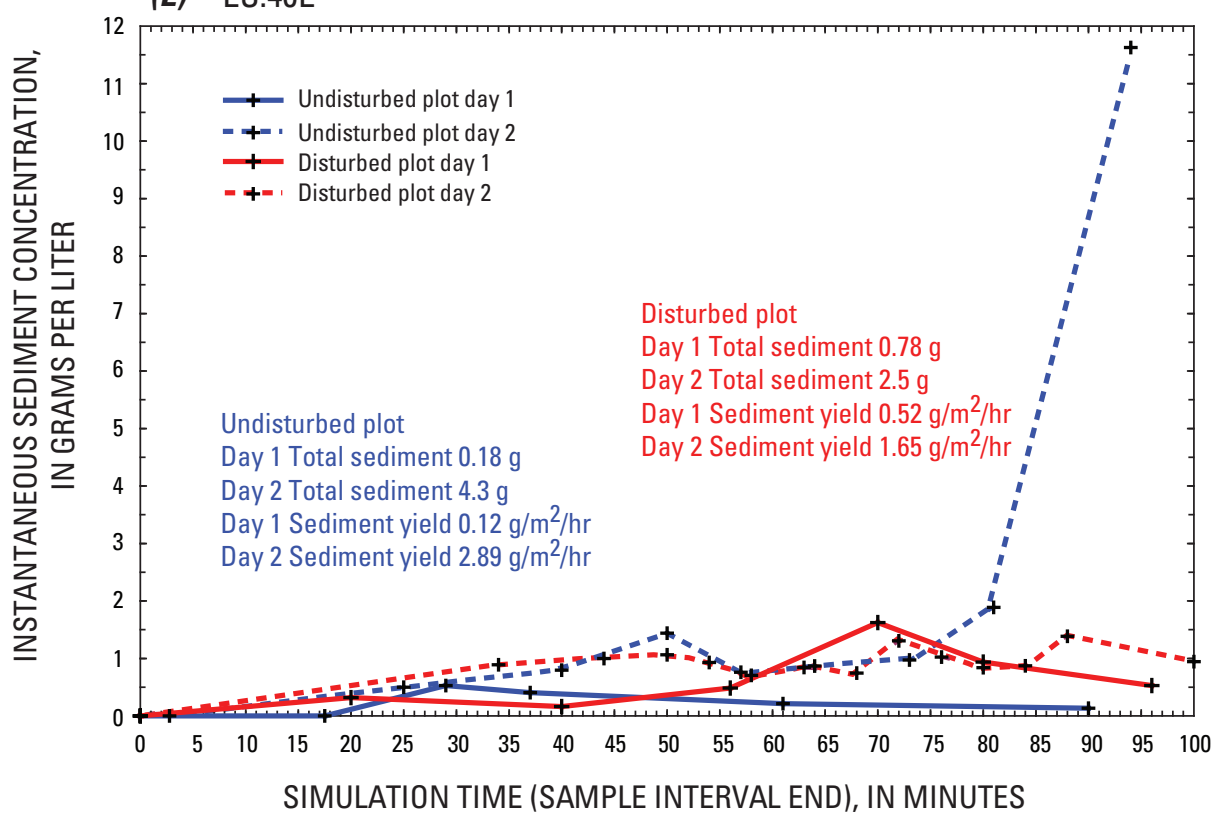

(F) ES.40NW

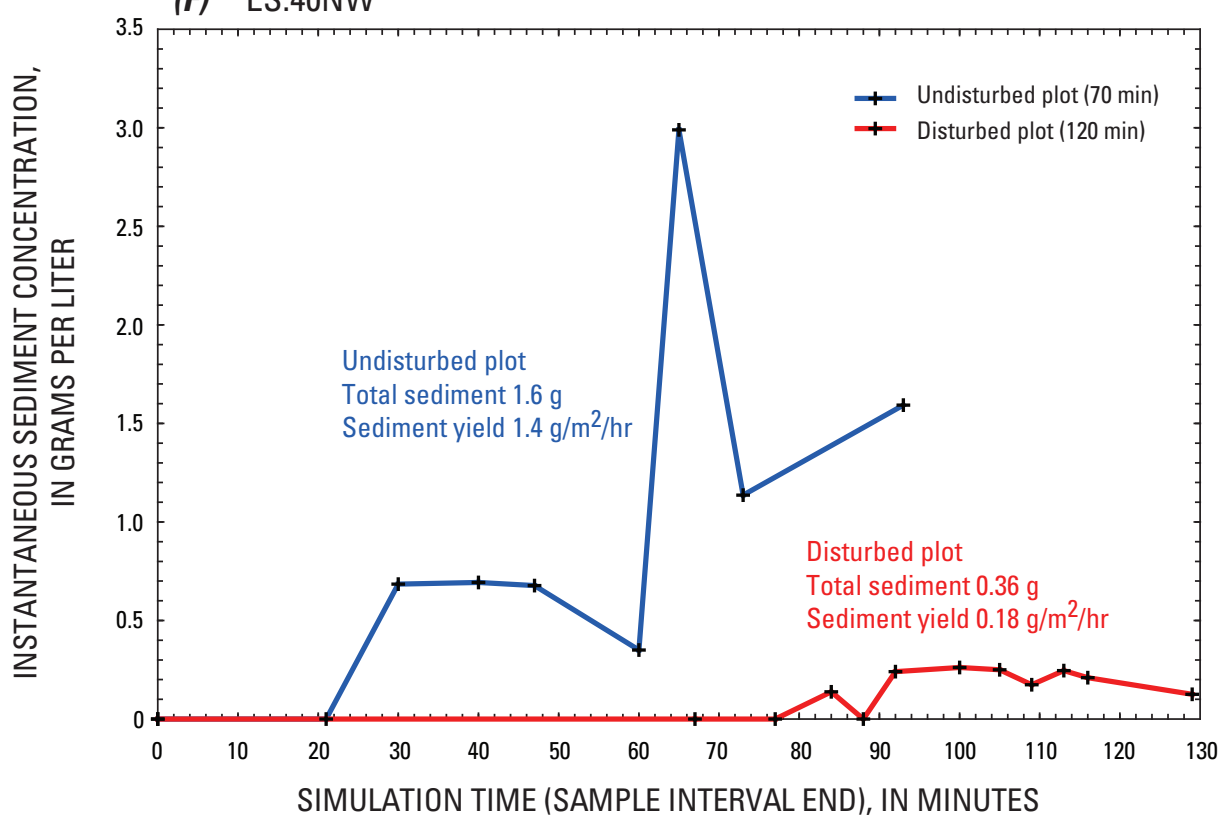

Figure 10. Sediment-concentration hydrographs from rainfall simulations.-Continued 
(G) CP.8S

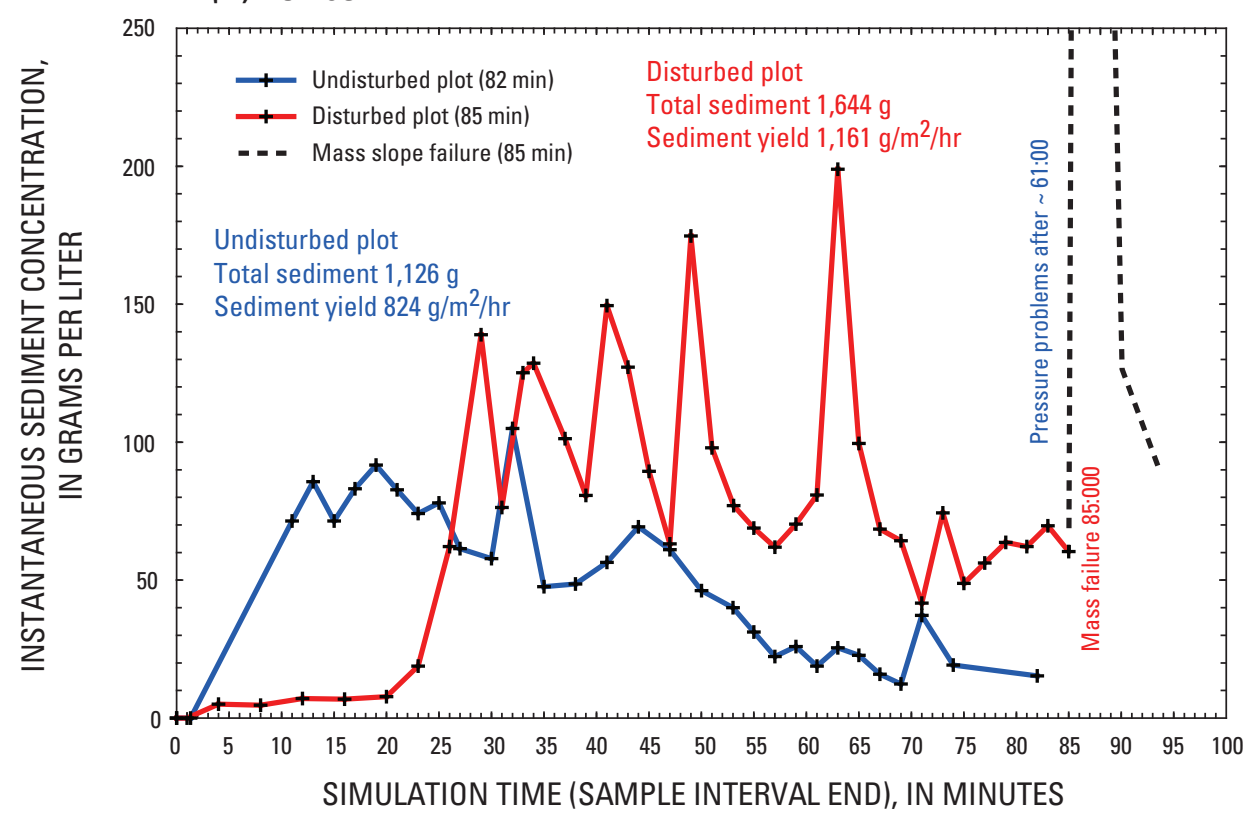

(H) CP.8W

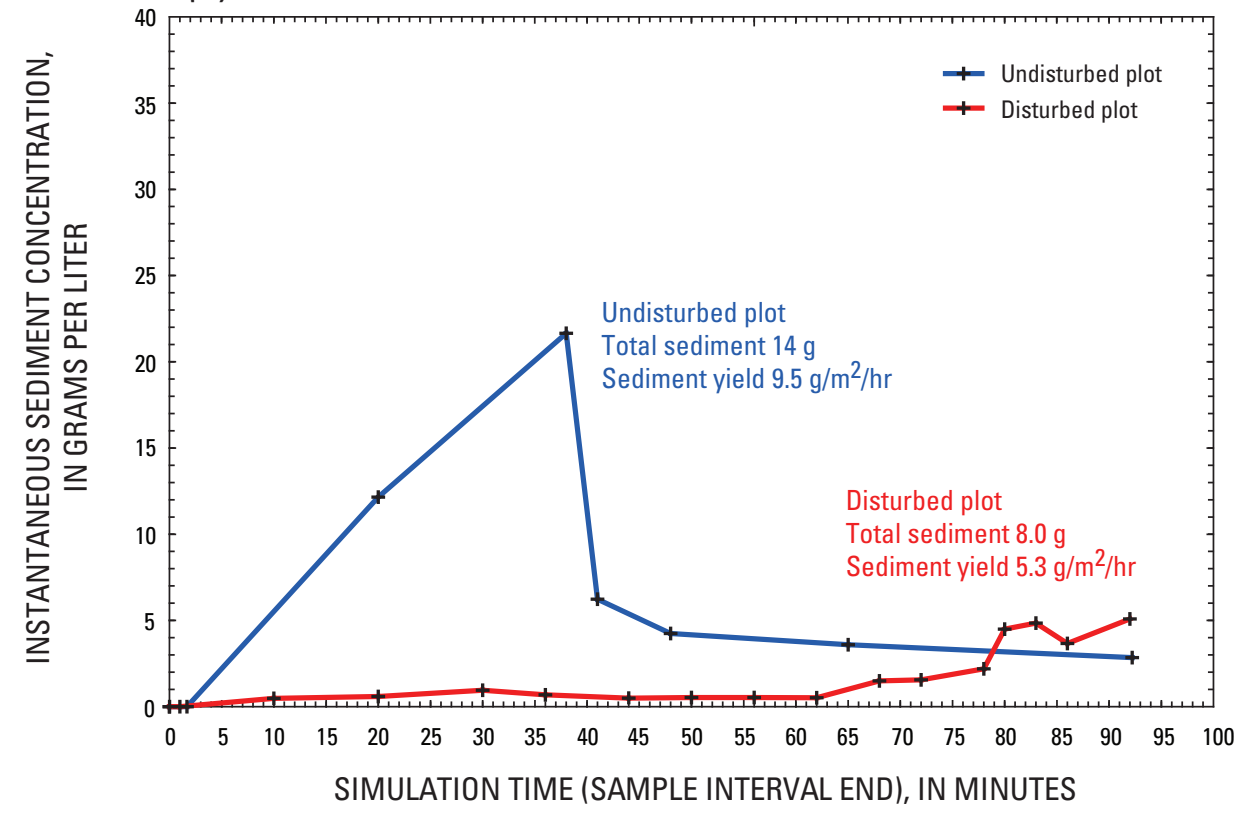

Figure 10. Sediment-concentration hydrographs from rainfall simulations. - Continued 

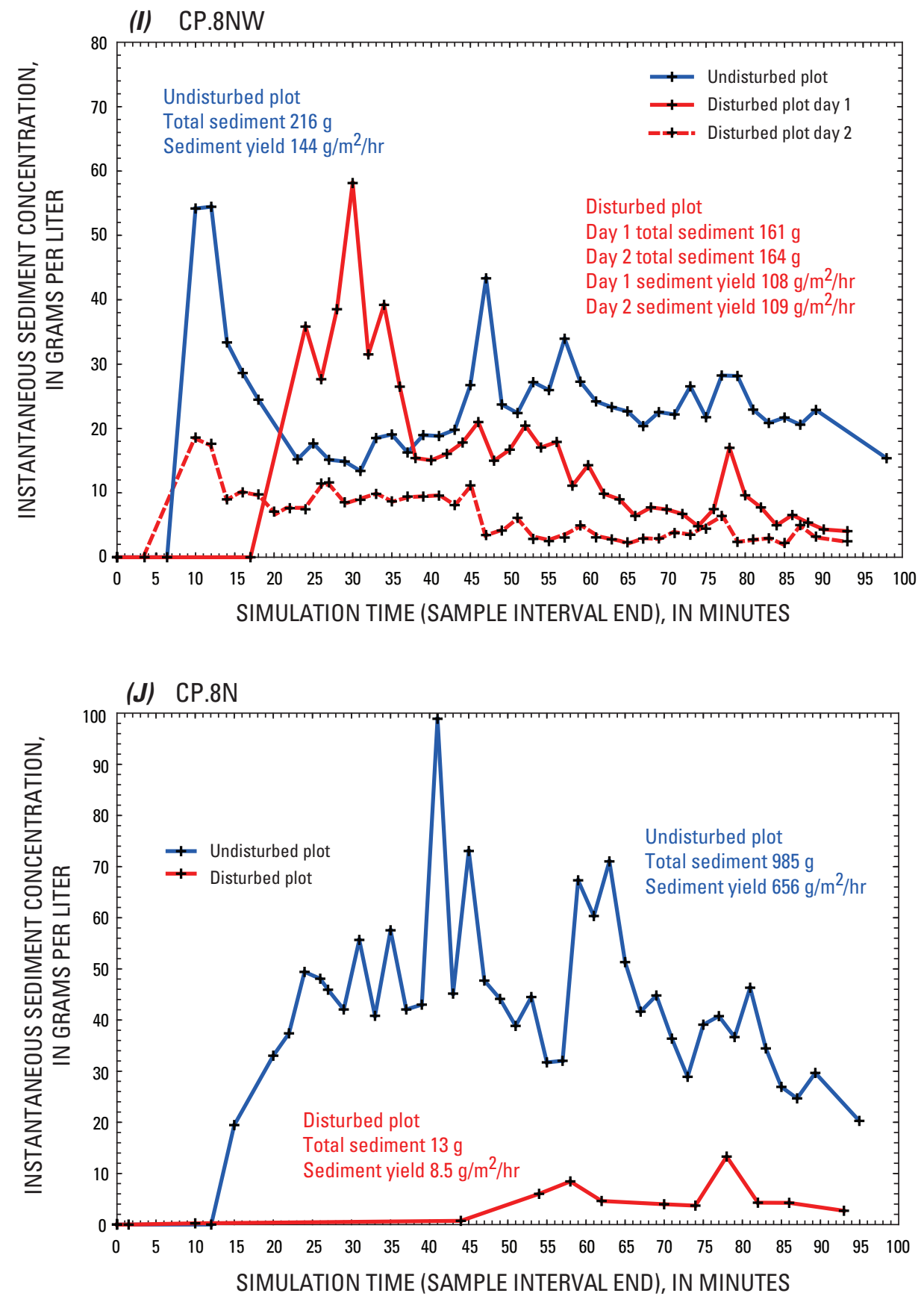

Figure 10. Sediment-concentration hydrographs from rainfall simulations.-Continued 
(K) CS.24E

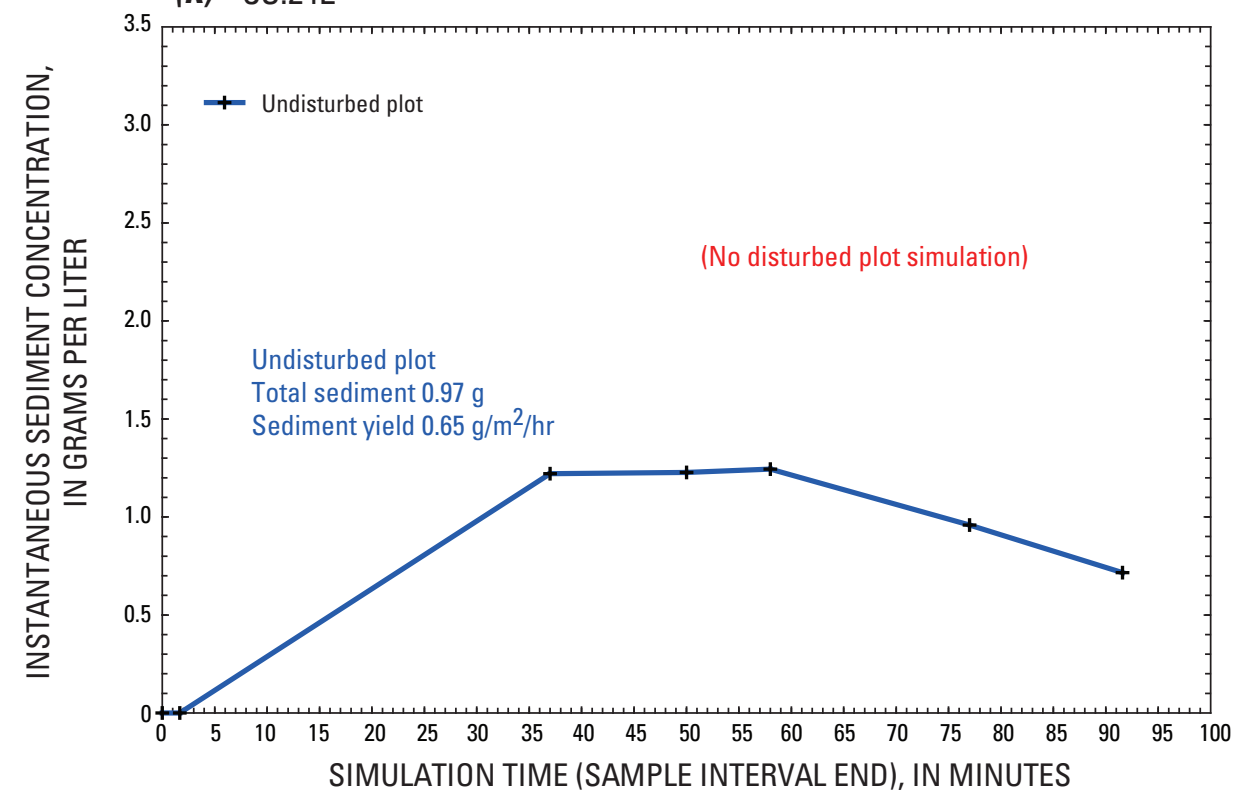

Figure 10. Sediment-concentration hydrographs from rainfall simulations.Continued

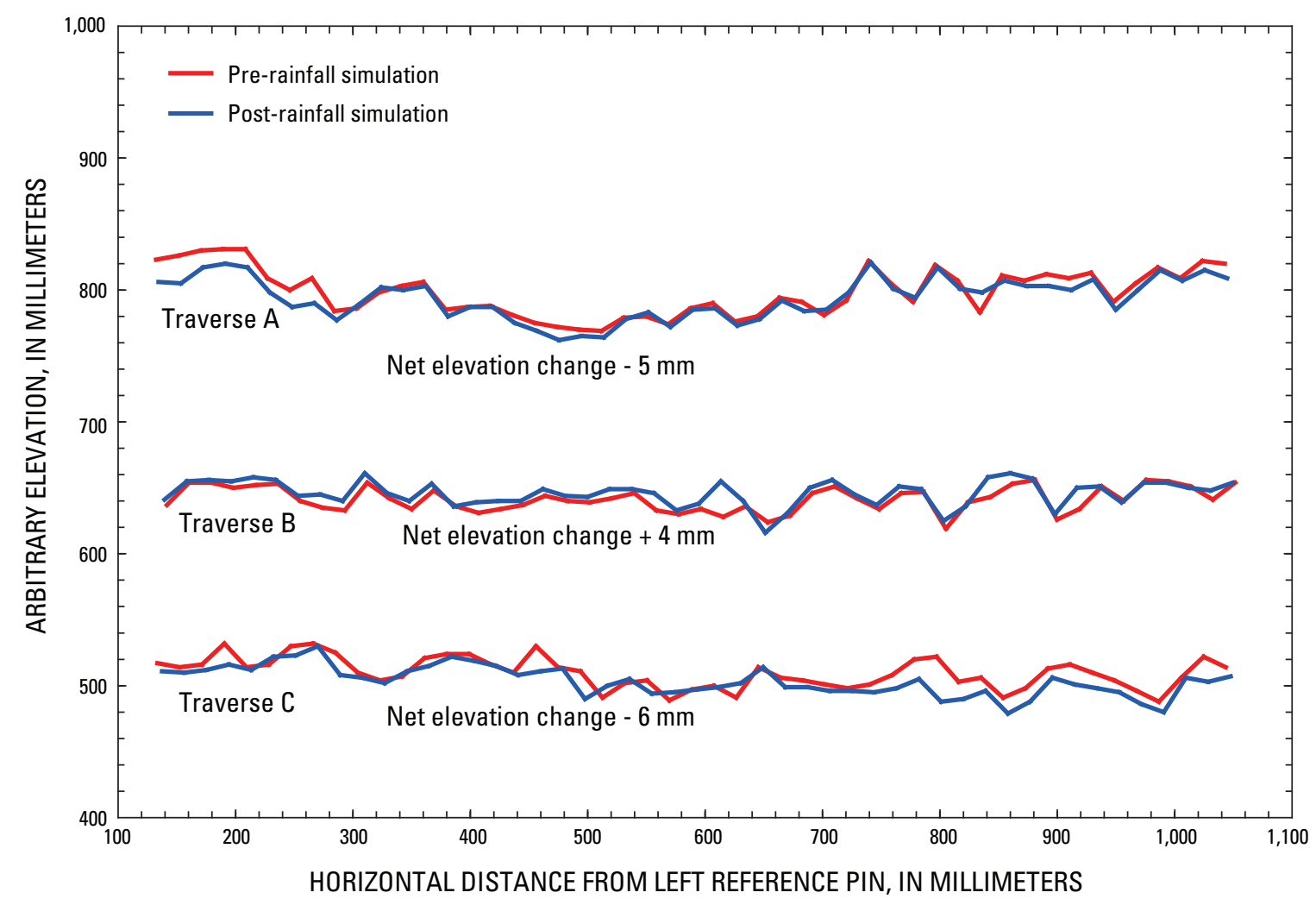

Figure 11. Soil-surface microtopography before and after a rainfall simulation, ES.9SE_U. 
Seasonal net hillslope-creep rates ranged from -13.7 to $108 \mathrm{~mm} / \mathrm{yr}$ at undisturbed (control) plots and from -4.87 to 164 $\mathrm{mm} / \mathrm{yr}$ at disturbed (treatment) plots (table 5). Annual net hillslope-creep rates ranged from -1.94 to $103 \mathrm{~mm} / \mathrm{yr}$ at undisturbed plots and from -2.17 to $18.4 \mathrm{~mm} / \mathrm{yr}$ at disturbed plots. Negative values (apparent upslope movement) could reflect soil swelling, initial or replicate measurement error, or long-term instability of the reference-mark (steel concrete-form stake) position due to deeper soil-horizon creep. Determination of deeper soil-horizon creep was beyond the scope of this report.

Hillslope-erosion plots were located in the upper Elephant Skin Wash study area on east, southeast, and northwest hillslope aspects (fig. 2), and in the Candy Lane study area on south, west, and north hillslope aspects (fig. 3). The erosion plots ranged in size from $4 \times 7 \mathrm{~m}\left(28 \mathrm{~m}^{2}\right)$ to $5 \times 10 \mathrm{~m}\left(50 \mathrm{~m}^{2}\right)$. Sediment trapped behind the silt fence downslope from each erosion plot was collected and removed periodically, weighed onsite, air dried in the sediment laboratory, and reweighed to determine the sediment yield (megagrams per hectare) and seasonal erosion rate (megagrams per hectare per year) (fig. 7, table 6 at the end of this report). All hillslope-erosion plots were located on undisturbed areas; none had been disturbed previously by OHV use.

Sediment yields and seasonal erosion rates were calculated over several month intervals for: (1) fall and winter 2004/2005, (2) spring 2005, (3) summer 2005, and (4) fall and winter 2005/2006. Because of nonconcurrent sampling dates at the upper Elephant Skin Wash and Candy Lane study areas, seasonal durations varied slightly from site to site. In addition to collecting captured sediment from the silt-fence traps, onsite observations were recorded as to the dominant hillslope-erosion mechanism. These mechanisms included gravity-induced soil-clod detachment and dry-ravel transport, and runoff-induced sheet wash (nonchannelized) and rill (channelized) erosion.

Sediment yields ranged from zero during the fall and winter of 2004/2005 to $12.2 \mathrm{Mg} / \mathrm{ha}$ during the summer of 2005. Seasonal erosion rates ranged from zero during the fall and winter of $2004 / 2005$ to $32.2 \mathrm{Mg} / \mathrm{ha} / \mathrm{yr}$ during the summer of 2005 . The highest sediment yields and erosion rates were observed on plot ES.9NW after channelized rills formed within the plot. For plots where rills did not form during the monitoring period, the maximum seasonal sediment yield was $6.80 \mathrm{Mg} / \mathrm{ha}$, and the maximum seasonal erosion rate was $14.3 \mathrm{Mg} / \mathrm{ha} / \mathrm{yr}$ during the summer of 2005.

Precipitation was measured at the upper Elephant Skin Wash and Candy Lane study areas from October 2004 through April 2006 and was compared to precipitation data from the Montrose \#2 climate station (National Oceanic and Atmospheric Administration, 2007). Precipitation data collected at the two Mancos Shale study areas provided a means to determine the magnitude and duration of individual storms as well as the total precipitation during intervals between the hillslope-creep and hillslope-erosion (silt-fence) measurements. Precipitation data from the Montrose \#2 climate station was compared to data from the upper Elephant Skin Wash and Candy Lane precipitation gages to determine site-to-site correlations and to provide study-site precipitation estimates for periods before installation of the study area precipitation gages in October 2004 when hillslope-creep measurements were made. Daily and cumulative daily precipitation data from the three gages are presented in table 7 at the end of this report. Cumulative daily precipitation curves are presented in figure 12.

\section{References Cited}

Benavides-Solorio, Juan, and MacDonald, L.H., 2001, Post-fire runoff and erosion from simulated rainfall on small plots, Colorado Front Range: Hydrologic Processes, v. 15, p. 2931-2952.

Byars, B.W., Allen, P.M., and Bingham, N.L., 1996, A portable rainfall simulator for assessing infiltration: Journal of Soil and Water Conservation, v. 51, no. 6, p. 508-510.

Frasier, G.W., Weltz, M., and Weltz, 1998, Rainfall simulator runoff hydrograph analysis: Journal of Range Management, v. 51, no. 5, p. 531-535.

Godfrey, A.E., 1997, Mass movement of Mancos Shale crust near Caineville, Utah -- a 30-year record: Geografiska Annaler, v. 79 A, p. $185-194$.

Guy, H.P., 1969, Laboratory theory and methods for sediment analysis: Techniques of Water-Resources Investigations of the U.S. Geological Survey, book 5, chap. C1, 58 p.

Hack, J.T., and Goodlett, J.C., 1960, Geomorphology and forest ecology of mountain regions in the central Appalachians: U.S. Geological Survey Professional Paper 347, 66 p.

Kellogg, K.S., 2004, The geologic story of the Gunnison Gorge National Conservation Area, Colorado: U.S. Geological Survey Professional Paper 1699, 40 p. 
Miller, J.F., Frederick, R.H., and Tracey, R.J., 1973, NOAA Atlas 2, Precipitation-frequency atlas of the western United States, volume 3-Colorado: Silver Spring, Md., U.S. Department of Commerce, National Oceanic and Atmospheric Administration, National Weather Service, 67 p.

Moody, J.A., and Martin, D.A., 2001, Hydrologic and sedimentologic response of two burned drainage basins in Colorado: U.S. Geological Survey Water-Resources Investigations Report 01-4122, variously paginated.

Munsell Color, 1992 (rev. ed.), Munsell soil color charts: Newburgh, N.Y., variously paginated.

National Oceanic and Atmospheric Administration, 2007, Precipitation data from Montrose \#2 Airport Station: Information available on Web, accessed February 7, 2007, at http://cdo.ncdc.noaa.gov/dly/DLY

Robichaud, P.R., and Brown, R.E., 2002, Silt fences-An economical technique for measuring hillslope soil erosion: U.S. Department of Agriculture, Forest Service, General Technical Report RMR-GTR-94, 24 p.

Ruhe, R.V., and Walker, P.H., 1968, Hillslope models and soil formation-I, open systems: Transactions of Ninth International Congress of Soil Science, v. 4, p. 551-560.

Tuttle, M. L.W., Fahy, Juli, Grauch, R.I., Ball, B.A., Chong, G.W., Elliott, J.G., Kosovich, J.J., Livo, K.E., and Stillings, L.L., 2007, Results of chemical analyses of soil, shale and soil/shale extract from the Mancos Shale Formation in the Gunnison Gorge National Conservation Area, southwestern Colorado, and at Hanksville, Utah: U.S. Geological Survey Open-File Report 2007-1002D, 24 p.

Wilcox, B.P., Wood, M.K., Tromble, J.T., and Ward, T.J., 1986, A hand-portable single nozzle rainfall simulator designed for use on steep slopes: Journal of Range Management, v. 39, no. 4, p. 375-377.

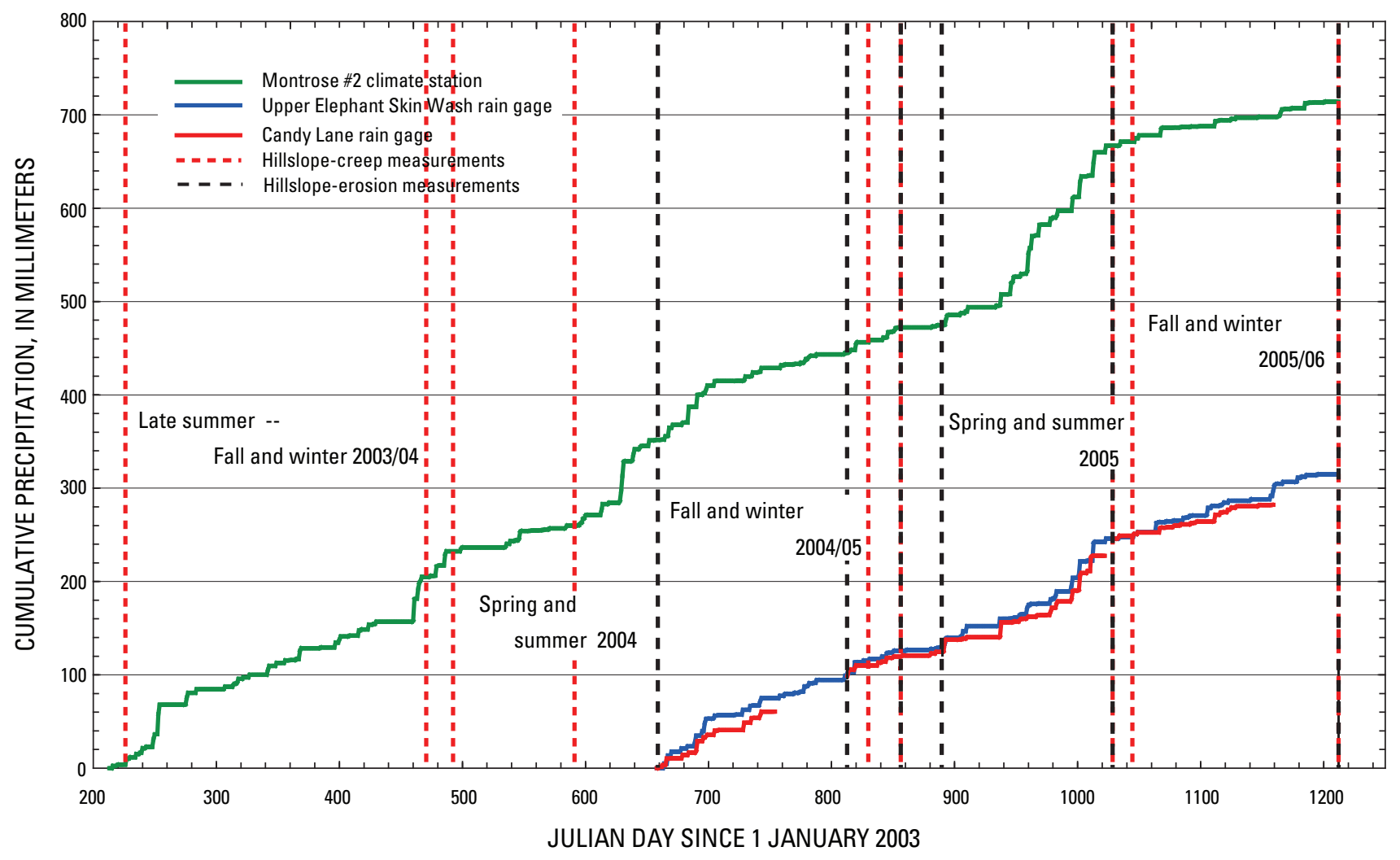

Figure 12. Cumulative precipitation at Montrose \#2 climate station, upper Elephant Skin Wash, and Candy Lane precipitation gages. 


\section{Supplemental Information: Tables}


Table 1. Sampling-site locations in upper Elephant Skin Wash study area, Gunnison Gorge National Conservation Area, southwestern Colorado.

[UTM, Universal Transverse Mercator projection, Zone $13 \mathrm{~N}$ coordinates (meters), North American Datum of 1983; UTM, Universal Transverse Mercator projection; GPS, global positioning system; OPUS, National Geodetic Survey Online Positioning Users Service; USGS, U.S. Geological Survey; LIDAR, light detecting and ranging; BM, bench mark; RM, reference mark; Sta, survey station number; U, undisturbed plot; D, disturbed plot; m, meters; N, north; E, east; S, south; W, west; aprx, approximately]

\begin{tabular}{|c|c|c|c|c|c|c|c|c|}
\hline $\begin{array}{c}\text { Hillslope } \\
\text { or watershed } \\
\text { identification } \\
\text { code }\end{array}$ & $\begin{array}{c}\text { Bench mark } \\
\text { reference mark, } \\
\text { or site } \\
\text { identification } \\
\text { code } \\
\end{array}$ & $\begin{array}{c}\text { UTM } \\
\text { easting } \\
\text { (meters) }\end{array}$ & $\begin{array}{c}\text { UTM } \\
\text { northing } \\
\text { (meters) }\end{array}$ & $\begin{array}{c}\text { Elevation } \\
\text { (meters) }\end{array}$ & $\begin{array}{c}\text { Horizon- } \\
\text { tal } \\
\text { error } \\
\text { estimate } \\
\text { (meters) }\end{array}$ & $\begin{array}{c}\text { Date } \\
\text { of GPS } \\
\text { meas- } \\
\text { urement }\end{array}$ & Time & Remarks \\
\hline Bench mark: & & & & & & & & Aluminum USGS cap set in concrete \\
\hline Elephant Skin & BM-1 ES & $255,208.27$ & $4,271,051.84$ & $1,874.66$ & 0.01 & 30 Aug 05 & $16: 53$ & Coordinates and elevation determined by OPUS, August 2005 \\
\hline \multicolumn{9}{|c|}{ Survey reference marks: } \\
\hline Basin ES.9 & Hub-1 & $255,298.0$ & $4,271,117.0$ & $1,881.2$ & 5.0 & 29 Jly 03 & $10: 12$ & Rebar, valley floor near basin 9 alluvial fan head \\
\hline Basin ES.40 & Hub-2 & $255,093.0$ & $4,271,021.0$ & $1,874.2$ & 7.0 & 30 Jly 03 & $8: 58$ & Rebar, valley floor of basin 40 \\
\hline \multicolumn{9}{|c|}{ Hillslope survey monuments: } \\
\hline ES.9SE & Sta $0+00$ & $255,295.0$ & $4,271,206.0$ & $1,920.5$ & 5.0 & 29 Apr 03 & $9: 51$ & Rebar, top left, northwest corner of hillslope plot \\
\hline ES.9SE & Sta $2+00$ & $255,323.0$ & $4,271,167.0$ & $1,885.8$ & 8.0 & 29 Apr 03 & $12: 04$ & Rebar, southwest corner of hillslope plot \\
\hline ES.9NW & Sta $0+00$ & $255,386.0$ & $4,271,128.0$ & $1,911.4$ & 5.0 & 29 Apr 03 & $15: 07$ & Rebar, top right corner of hillslope plot \\
\hline ES.9NW & Sta $2+00$ & $255,341.0$ & $4,271,150.0$ & $1,884.0$ & 5.0 & 29 Apr 03 & $14: 51$ & Rebar, bottom right corner of hillslope plot \\
\hline ES.9SW & Sta $0+00$ & $255,263.0$ & $4,271,180.0$ & $1,912.3$ & 6.0 & 30 Jly 03 & $15: 14$ & Rebar, ridge crest motorcycle track \\
\hline ES.9SW & Sta $3+00$ & $255,228.0$ & $4,271,108.0$ & $1,878.2$ & 7.6 & 30 Jly 03 & $16: 20$ & Rebar, foot of hillslope \\
\hline ES.40NW & Sta $0+00$ & $255,098.0$ & $4,270,963.0$ & $1,891.0$ & 8.0 & 9 Jun 05 & & Rebar, top left of hillslope plot \\
\hline ES.40NW & Sta $0+60$ & $255,087.0$ & $4,270,975.0$ & $1,901.0$ & 7.0 & 6 Jun 05 & $17: 13$ & Rebar, bottom left of hillslope plot \\
\hline ES.40E & Sta $0+00$ & $255,021.0$ & $4,271,004.0$ & $1,895.9$ & 9.0 & 30 Apr 03 & $10: 17$ & Rebar, top right of hillslope plot \\
\hline ES.40E & Sta $1+00$ & $255,045.0$ & $4,270,998.0$ & $1,895.0$ & 5.0 & 27 Apr 05 & $12: 07$ & Rebar, bottom right of hillslope plot \\
\hline ES.38NE & none & $255,177.0$ & $4,270,974.0$ & $1,877.0$ & 5.0 & 26 Apr 06 & $11: 44$ & Carsonite post, top of "Elevator to Hell Trail" \\
\hline ES.38NE & none & $255,075.0$ & $4,270,819.0$ & $1,965.0$ & 5.0 & 26 Apr 06 & $12: 00$ & Carsonite post, bottom of "Elevator to Hell Trail" \\
\hline \multicolumn{9}{|c|}{ Photo monuments: } \\
\hline Basin ES.9 & PM-01 & $255,368.0$ & $4,271,113.0$ & $1,904.1$ & 7.0 & 29 Apr 03 & $12: 47$ & Wood stake on upper hillslope ES.9NW \\
\hline Basin ES.9 & PM-02 & $255,281.0$ & $4,271,188.0$ & $1,906.2$ & 4.0 & 29 Apr 03 & $17: 30$ & Wood stake on upper hillslope ES.9SE \\
\hline Basin ES.40 & PM-03 & $255,098.0$ & $4,270,980.0$ & $1,879.7$ & 5.0 & 30 Apr 03 & $12: 01$ & Wood stake, $15 \mathrm{~m}$ left (NE) of rainfall plot ES.40NW_D \\
\hline Basin ES.40 & PM-04 & $255,066.0$ & $4,270,982.0$ & $1,881.5$ & 4.0 & 30 Apr 03 & $16: 53$ & Wood stake \\
\hline Basin ES.8 & PM-07 & $255,310.0$ & $4,271,235.0$ & $1,927.9$ & 8.0 & 1 May 03 & $15: 32$ & Wired flag, east of ES.9NW Sat $0+00$ \\
\hline Basin ES.10 & PM-08 & $255,385.0$ & $4,271,102.0$ & $1,907.4$ & 6.0 & 1 May 03 & $16: 09$ & Wired flag, basin $10, W$ hillslope, near ridge crest \\
\hline Basin ES.10 & PM-09 & $255,487.0$ & $4,271,075.0$ & $1,913.2$ & 8.0 & 1 May 03 & $16: 50$ & Wired flag, basin 10 , E hillslope, near ridge crest \\
\hline Basin ES.11 & PM-10 & $255,518.0$ & $4,271,103.0$ & $1,917.5$ & 4.4 & 1 May 03 & $17: 07$ & Wired flag, basin 11 , W hillslope, near ridge crest \\
\hline
\end{tabular}


Table 1. Sampling-site locations in upper Elephant Skin Wash study area, Gunnison Gorge National Conservation Area, southwestern Colorado.-Continued

[UTM, Universal Transverse Mercator projection, Zone 13 N coordinates (meters), North American Datum of 1983; UTM, Universal Transverse Mercator projection; GPS, global positioning system; OPUS, National Geodetic Survey Online Positioning Users Service; USGS, U.S. Geological Survey; LIDAR, light detecting and ranging; BM, bench mark; RM, reference mark; Sta, survey station number; U, undisturbed plot; D, disturbed plot; m, meters; N, north; E, east; S, south; W, west; aprx, approximately]

\begin{tabular}{|c|c|c|c|c|c|c|c|c|}
\hline $\begin{array}{c}\text { Hillslope } \\
\text { or watershed } \\
\text { identification } \\
\text { code }\end{array}$ & $\begin{array}{c}\text { Bench mark } \\
\text { reference mark, } \\
\text { or site } \\
\text { identification } \\
\text { code } \\
\end{array}$ & $\begin{array}{c}\text { UTM } \\
\text { easting } \\
\text { (meters) }\end{array}$ & $\begin{array}{c}\begin{array}{c}\text { UTM } \\
\text { northing } \\
\text { (meters) }\end{array} \\
\end{array}$ & $\begin{array}{c}\text { Elevation } \\
\text { (meters) }\end{array}$ & $\begin{array}{c}\text { Horizon- } \\
\text { tal } \\
\text { error } \\
\text { estimate } \\
\text { (meters) }\end{array}$ & $\begin{array}{c}\text { Date } \\
\text { of GPS } \\
\text { meas- } \\
\text { urement }\end{array}$ & Time & Remarks \\
\hline \multicolumn{8}{|c|}{ Rainfall - runoff simulation plots: } & Elevation from bare earth LIDAR, January 2006 \\
\hline ES.9SE & ES.9SE_U & $255,329.0$ & $4,271,173.0$ & $1,890.9$ & 6.0 & 27 Apr 05 & $13: 48$ & On plot \\
\hline ES.9SE & ES.9SE_D & $255,313.0$ & $4,271,159.0$ & $1,888.4$ & 6.0 & $27 \mathrm{Apr} 05$ & $14: 05$ & On plot \\
\hline ES.9NW & ES.9NW_U & $255,334.0$ & $4,271,143.0$ & $1,887.6$ & 8.0 & 27 Apr 05 & $14: 26$ & On plot \\
\hline ES.9NW & ES.9NW_D & $255,332.0$ & $4,271,133.0$ & $1,888.0$ & 6.0 & 27 Apr 05 & $14: 51$ & On plot \\
\hline ES.9SW & ES.9SW_U & $255,221.0$ & $4,271,107.0$ & $1,878.3$ & 4.0 & 27 Apr 05 & $15: 22$ & On plot \\
\hline ES.9SW & ES.9SW_D & $255,225.0$ & $4,271,105.0$ & $1,878.5$ & 4.0 & 27 Apr 05 & $15: 48$ & On plot \\
\hline ES.38NE & ES.38NE_U & $255,168.0$ & $4,270,966.0$ & $1,879.2$ & 5.0 & 27 Apr 05 & $10: 55$ & GPS between $\mathrm{U}(2.5 \mathrm{~m}$ right $)$ and $\mathrm{D}(2.5 \mathrm{~m}$ left $)$ plots \\
\hline ES.38NE & ES.38NE_D & $255,172.0$ & $4,270,962.0$ & $1,879.2$ & 5.0 & 27 Apr 05 & $10: 55$ & GPS between $\mathrm{U}(2.5 \mathrm{~m}$ right $)$ and $\mathrm{D}(2.5 \mathrm{~m}$ left $)$ plots \\
\hline ES.40E & ES.40E_U & $255,045.0$ & $4,270,998.0$ & $1,887.2$ & 5.0 & 27 Apr 05 & $12: 07$ & At Sta $1+00$ rebar, just below ES.40E_U plot \\
\hline ES.40E & ES.40E_D & $255,050.0$ & $4,271,008.0$ & $1,885.9$ & 6.0 & 27 Apr 05 & $12: 30$ & Just below ES.40E_D plot \\
\hline ES.40NW & ES.40NW_U & $255,087.0$ & $4,270,972.0$ & $1,882.2$ & 6.0 & 6 Jun 05 & $17: 26$ & On plot \\
\hline ES.40NW & ES.40NW_D & $255,090.0$ & $4,270,976.0$ & $1,885.4$ & 7.0 & 9 Jun 05 & $8: 19$ & On lower scaffold plank, left end \\
\hline \multicolumn{9}{|l|}{ Rain gage: } \\
\hline Elephant Skin & ES-RG1 & $255,243.0$ & $4,270,999.0$ & $1,880.0$ & 6.71 & 19 Oct 04 & $11: 00$ & Upstream from uppermost, cross-valley dam \\
\hline \multicolumn{9}{|c|}{ Hillslope erosion silt fence: } \\
\hline ES.9SE & none & $255,337.2$ & $4,271,178.8$ & $1,889.2$ & 9.14 & 20 Oct 04 & $16: 55$ & At silt fence, plot size $5 \times 10 \mathrm{~m}$ \\
\hline ES.9NW & none & $255,342.1$ & $4,271,147.1$ & $1,895.6$ & 11.89 & 21 Oct 04 & 12:01 & At silt fence, plot size $5 \times 10 \mathrm{~m}$ \\
\hline ES.40E & none & $255,038.0$ & $4,270,985.0$ & $1,892.0$ & 7.00 & 7 Jun 05 & $17: 17$ & At silt fence, plot size $4 \times 7 \mathrm{~m}$ \\
\hline
\end{tabular}


Table 2. Sampling-site locations in Candy Lane study area, Gunnison Gorge National Conservation Area, southwestern Colorado.

[UTM, Universal Transverse Mercator projection, Zone 13 N coordinates (meters), North American Datum of 1983; UTM, Universal Transverse Mercator projection; GPS, global positioning system; OPUS, National Geodetic Survey Online Positioning Users Service; USGS, U.S. Geological Survey; LIDAR, light detecting and ranging; BM, bench mark; RM, reference mark; Sta, survey station number; U, undisturbed plot; D, disturbed plot; m, meters; N, north; E, east; S, south; W, west; aprx, approximately]

\begin{tabular}{|c|c|c|c|c|c|c|c|c|}
\hline $\begin{array}{c}\text { Hillslope } \\
\text { or watershed } \\
\text { identification } \\
\text { code }\end{array}$ & $\begin{array}{c}\text { Bench mark } \\
\text { reference mark, } \\
\text { or site } \\
\text { identification } \\
\text { code }\end{array}$ & $\begin{array}{c}\text { UTM } \\
\text { easting } \\
\text { (meters) }\end{array}$ & $\begin{array}{c}\text { UTM } \\
\text { northing } \\
\text { (meters) }\end{array}$ & $\begin{array}{l}\text { Elevation } \\
\text { (meters) }\end{array}$ & $\begin{array}{l}\text { Horizon- } \\
\text { tal } \\
\text { error } \\
\text { estimate } \\
\text { (meters) }\end{array}$ & $\begin{array}{c}\text { Date } \\
\text { of GPS } \\
\text { meas- } \\
\text { urement }\end{array}$ & Time & Remarks \\
\hline $\begin{array}{l}\text { Bench mark: } \\
\text { Candy Lane }\end{array}$ & $\mathrm{BM}-1 \mathrm{CL}$ & $247,360.29$ & $4,278,124.61$ & $1,755.04$ & 0.02 & 31 Aug 05 & $13: 51$ & $\begin{array}{l}\text { Aluminum USGS cap set in concrete } \\
\text { OPUS solution August } 2005\end{array}$ \\
\hline \multicolumn{9}{|c|}{ Survey reference marks: } \\
\hline Basin CP.8 & Hub-1 & $247,499.0$ & $4,278,297.0$ & $1,759.0$ & 5.2 & 27 Apr 04 & $9: 58$ & Rebar on ridge between $\mathrm{S}$ - and $\mathrm{N}$-facing hillslopes \\
\hline Basin CS.24 & Hub-3 & $247,178.0$ & $4,278,023.0$ & $1,747.0$ & 4.6 & 27 Apr 04 & $16: 48$ & Rebar on valley floor \\
\hline Basin CS.24 & RM-5 & $247,131.0$ & $4,278,023.0$ & $1,748.0$ & 5.2 & 27 Apr 04 & $16: 58$ & Boulder with chisled "X" mark \\
\hline Basin CS.24 & RM-6 & $247,155.0$ & $4,277,978.0$ & $1,747.0$ & 6.4 & 27 Apr 04 & $17: 13$ & Boulder with chisled "X" mark \\
\hline Basin CS.24 & SW corner & $247,213.0$ & $4,278,029.0$ & $1,743.0$ & 4.3 & 27 Apr 04 & $17: 23$ & SW corner post on exclosure area \\
\hline Basin CS.24 & NW corner & $247,172.5$ & $4,278,152.6$ & $1,739.2$ & 4.3 & 27 Apr 04 & $17: 28$ & NW corner post, on exclosure area \\
\hline Basin CS.24 & RM-4 NE cornr & $247,224.0$ & $4,278,175.0$ & $1,742.0$ & 4.0 & 27 Apr 04 & $17: 31$ & NE corner post on exclosure area \\
\hline Basin CS.24 & SE corner & $247,266.7$ & $4,278,053.3$ & $1,742.8$ & 4.3 & 27 Apr 04 & $17: 45$ & SE corner post on exclosure area \\
\hline \multicolumn{9}{|c|}{ Hillslope survey monuments: } \\
\hline CP.8S & Sta $0+00$ & $247,487.0$ & $4,278,391.0$ & $1,788.0$ & 4.9 & 27 Apr 04 & $10: 37$ & Rebar, top of hillslope \\
\hline CP.8S & Sta $2+50$ & $247,485.0$ & $4,278,326.0$ & $1,759.0$ & 4.6 & 27 Apr 04 & $12: 17$ & Rebar, foot of hillslope \\
\hline CP.8W & Sta $0+00$ & $247,579.0$ & $4,278,376.0$ & $1,798.0$ & 5.2 & 27 Apr 04 & $11: 23$ & Rebar, top of hillslope \\
\hline CP.8W & Sta $3+00$ & $247,514.0$ & $4,278,337.0$ & $1,751.0$ & 4.3 & 27 Apr 04 & $12: 23$ & Rebar, foot of hillslope \\
\hline CP.8NW & Sta $0+00$ & $247,572.0$ & $4,278,272.0$ & $1,790.0$ & 4.0 & 24 Apr 06 & $13: 47$ & Rebar, top of hillslope \\
\hline CP.8NW & Sta $3+26.5$ & $247,518.0$ & $4,278,342.0$ & $1,741.0$ & 7.0 & 24 Apr 06 & $13: 37$ & Rebar, foot of hillslope \\
\hline CP.8N & Sta $0+00$ & $247,473.0$ & $4,278,134.0$ & $1,789.0$ & 4.6 & 27 Apr 04 & $12: 52$ & Rebar, top of hillslope \\
\hline CP.8N & Sta $3+10$ & $247,472.0$ & $4,278,214.0$ & $1,760.0$ & 4.3 & 27 Apr 04 & $13: 57$ & Rebar, foot of hillslope \\
\hline CS.24E & Sta $0+00$ & $247,106.0$ & $4,277,997.0$ & $1,762.0$ & 4.3 & 27 Apr 04 & $16: 32$ & Rebar, top of hillslope \\
\hline CS.24E & Sta $2+20$ & $247,169.0$ & $4,278,003.0$ & $1,731.0$ & 5.0 & 24 Apr 06 & $16: 38$ & Rebar, foot of hillslope \\
\hline
\end{tabular}


Table 2. Sampling-site locations in Candy Lane study area, Gunnison Gorge National Conservation Area, southwestern Colorado.-Continued

[UTM, Universal Transverse Mercator projection, Zone 13 N coordinates (meters), North American Datum of 1983; UTM, Universal Transverse Mercator projection; GPS, global positioning system; OPUS, National Geodetic Survey Online Positioning Users Service; USGS, U.S. Geological Survey; LIDAR, light detecting and ranging; BM, bench mark; RM, reference mark; Sta, survey station number; U, undisturbed plot; D, disturbed plot; m, meters; N, north; E, east; S, south; W, west; aprx, approximately]

\begin{tabular}{|c|c|c|c|c|c|c|c|c|}
\hline $\begin{array}{c}\text { Hillslope } \\
\text { or watershed } \\
\text { identification } \\
\text { code }\end{array}$ & $\begin{array}{l}\text { Bench mark } \\
\text { reference mark, } \\
\text { or site } \\
\text { identification } \\
\text { code }\end{array}$ & $\begin{array}{c}\text { UTM } \\
\text { easting } \\
\text { (meters) }\end{array}$ & $\begin{array}{c}\text { UTM } \\
\text { northing } \\
\text { (meters) }\end{array}$ & $\begin{array}{c}\text { Elevation } \\
\text { (meters) }\end{array}$ & $\begin{array}{l}\text { Horizon- } \\
\text { tal } \\
\text { error } \\
\text { estimate } \\
\text { (meters) }\end{array}$ & $\begin{array}{c}\text { Date } \\
\text { of GPS } \\
\text { meas- } \\
\text { urement }\end{array}$ & Time & Remarks \\
\hline
\end{tabular}

Photo monument:

Basin CL.8

PM-01

$247,243.0$

$4,278,213.0$

$1,741.0$

$4.3 \quad 27 \mathrm{Apr} 04$

$17: 38$

Earth mound near Candy Lane road

Rainfall - runoff simulation plots:

$\begin{array}{ll}\text { CP.8S } & \text { CP.8S_U } \\ \text { CP.8S } & \text { CP.8S_D }\end{array}$

CP.8W

CP.8W_U

$247,475.0$

$4,278,349.0 \quad 1,748.3$

$247,480.0$

$4,278,349.0$

$1,747.5$

4.6 27 Apr 04

12:17 $23 \mathrm{~m} \mathrm{~N}, 10 \mathrm{~m} \mathrm{~W}$ of waypoint 59 , Sta $2+50$

CP.8W

$247,531.0$

$4,278,357.0$

$1,752.2$

$4.627 \mathrm{Apr} 04$

$12: 17$

$3 \mathrm{~m} \mathrm{~N}, 5 \mathrm{~m} \mathrm{~W}$ of waypoint 59

4.3 27 Apr 04

$12: 23$

CP. 8 NW

$\operatorname{crs} 2 \sin U$

$247,539.0$

$4,278,344.0 \quad 1,753.9$

$4.3 \quad 27$ Apr 04

$12: 23$

$25 \mathrm{~m} \mathrm{E}, 9 \mathrm{~m} \mathrm{~N}$ of waypoint 60 , Sta $3+00$

CP.8NW

CP.8NW_D1

$247,522.0$

$4,278,327.0$

$1,755.0$

$4,278,324.0 \quad 1,752.4$

$5.0 \quad 2$ May 05

$8: 22$

CP.8N

CP.8N_U

$247,478.0$

$4,278,201.0$

$1,752.8$

CP.8N

$247,475.0$

$4,278,201.0$

$1,752.5$

CS.24E

CS.24E_U

$247,143.0$

$4,277,997.2$

$1,736.5$

$8: 40$

On scaffold plank below plot

On lower left corner of plot

9.0 3 Apr 05 18:35 On plot

none $\quad 3$ Apr $05 \quad 18: 25 \quad$ On plot

Rain gage:

Basin CF

CL-RG1

$247,474.0$

$4,278,254.0$

$1,743.0$

4.0 3 Apr 05

18:12 Relocated to here 23 March 2005

Hillslope erosion silt fence:

CP.8S none

CP.8W none

$247,488.0$

$4,278,357.0$

$1,771.0$

$247,531.0$

$4,278,382.0$

$1,777.0$

CP.8N

none

$247,470.0$

$4,278,202.0 \quad 1,751.0$

$\begin{array}{ll}5.0 & 6 \text { May } 05 \\ 6.0 & 5 \text { May } 05\end{array}$

9:30 At silt fence, plot size $4 \times 7 \mathrm{~m}$

6.0 5 May $05 \quad 17: 21 \quad 5 \mathrm{~m}$ downslope from fence, plot size $5 \times 10 \mathrm{~m}$

5.0 3 Apr $05 \quad 18: 22$ At silt fence, plot size $5 \times 10 \mathrm{~m}$ 
Table 2. Sampling-site locations in Candy Lane study area, Gunnison Gorge National Conservation Area, southwestern Colorado.-Continued

[UTM, Universal Transverse Mercator projection, Zone $13 \mathrm{~N}$ coordinates (meters), North American Datum of 1983; UTM, Universal Transverse Mercator projection; GPS, global positioning system; OPUS, National Geodetic Survey Online Positioning Users Service; USGS, U.S. Geological Survey; LIDAR, light detecting and ranging; BM, bench mark; RM, reference mark; Sta, survey station number; U, undisturbed plot; D, disturbed plot; m, meters; N, north; E, east; S, south; W, west; aprx, approximately]

\begin{tabular}{|c|c|c|c|c|c|c|c|c|}
\hline $\begin{array}{c}\text { Hillslope } \\
\text { or watershed } \\
\text { identification } \\
\text { code }\end{array}$ & $\begin{array}{c}\text { Bench mark } \\
\text { reference mark, } \\
\text { or site } \\
\text { identification } \\
\text { code } \\
\end{array}$ & $\begin{array}{c}\begin{array}{c}\text { UTM } \\
\text { easting } \\
\text { (meters) }\end{array} \\
\end{array}$ & $\begin{array}{c}\text { UTM } \\
\text { northing } \\
\text { (meters) }\end{array}$ & $\begin{array}{c}\text { Elevation } \\
\text { (meters) }\end{array}$ & $\begin{array}{c}\text { Horizon- } \\
\text { tal } \\
\text { error } \\
\text { estimate } \\
\text { (meters) }\end{array}$ & $\begin{array}{c}\text { Date } \\
\text { of GPS } \\
\text { meas- } \\
\text { urement }\end{array}$ & Time & Remarks \\
\hline \multicolumn{9}{|c|}{ Arroyo survey reference marks: } \\
\hline CL Arroyo & RM-1, PM & $246,886.0$ & $4,279,535.0$ & $1,745.0$ & 4.9 & 28 Apr 04 & $9: 38$ & Rebar on pediment aprx $300 \mathrm{~m} \mathrm{NW}$ of headcut \\
\hline CL Arroyo & Hub-1, PM & $246,985.0$ & $4,279,515.0$ & $1,724.0$ & 4.9 & 28 Apr 04 & $10: 39$ & Rebar on 3rd arroyo dam aprx $80 \mathrm{~m}$ bel headcut \\
\hline CL Arroyo & RM-4 & $247,125.0$ & $4,279,381.0$ & $1,735.0$ & 4.9 & 28 Apr 04 & $10: 30$ & Rebar on low round hill E of arroyo \\
\hline CL Arroyo & Grid North & $246,990.0$ & $4,279,606.0$ & $1,724.0$ & 5.0 & 25 Apr 06 & $8: 55$ & Rebar \\
\hline CL Arroyo & LHP-1 & $246,950.0$ & $4,279,461.0$ & $1,716.0$ & 4.6 & 28 Apr 04 & $14: 14$ & Rebar, arroyo cross section 1 \\
\hline CL Arroyo & RHP-1 & $247,039.0$ & $4,279,443.0$ & $1,724.0$ & 6.0 & 25 Apr 06 & $9: 23$ & Rebar \\
\hline CL Arroyo & LHP-2 & $246,950.0$ & $4,279,479.0$ & $1,713.0$ & 4.6 & 28 Apr 04 & $14: 48$ & Rebar, arroyo cross section 2 \\
\hline CL Arroyo & RHP-2 & $247,035.0$ & $4,279,474.0$ & $1,723.0$ & 6.0 & 25 Apr 06 & 9:18 & Rebar \\
\hline CL Arroyo & LHP-3 & $246,932.3$ & $4,279,556.4$ & $1,714.0$ & 5.2 & 28 Apr 04 & $14: 52$ & Rebar, arroyo cross section 3 \\
\hline CL Arroyo & RHP-3 & $246,997.0$ & $4,279,549.0$ & $1,714.0$ & 4.6 & $28 \mathrm{Apr} 04$ & $15: 02$ & Rebar \\
\hline
\end{tabular}


Table 3. Rainfall-runoff and ancillary data from Gunnison Gorge National Conservation Area, southwestern Colorado.

[Sim., simulation; no., number; concn., concentration; Normal., normalized; rel $100 \mathrm{yr}$, variable is normalized to the 100-year rainfall rate of $38 \mathrm{~mm}$ per hour; \&, and; min, minute; $\mathrm{mm}$, millimeter, $\mathrm{mm} / \mathrm{h}$, millimeter per hour; $\mathrm{mL}$, milliliter; $\mathrm{mL} / \mathrm{m}^{2} / \mathrm{h}$, milliliter per square meter per hour; g/L, grams per liter; g, grams; g/m²/h; grams per square meter per hour; $\mathrm{mL} / \mathrm{h}$; milliliter per hour; na, not applicable or no data; Yellow highlighted fields are from second-day rainfall simulations]

\begin{tabular}{|c|c|c|c|c|c|c|c|c|c|c|c|c|c|}
\hline $\begin{array}{c}\text { Rainfall- } \\
\text { runoff } \\
\text { plot } \\
\text { identification } \\
\text { code } \\
\end{array}$ & $\begin{array}{c}\text { Sim } \\
\text { no. }\end{array}$ & $\begin{array}{c}\text { Hillslope } \\
\text { aspect } \\
\text { (degrees) }\end{array}$ & $\begin{array}{c}\text { Slope } \\
\text { length } \\
\text { above } \\
\text { plot } \\
\text { (meters) }\end{array}$ & $\begin{array}{c}\text { Surveyed } \\
\text { hillslope } \\
\text { gradient } \\
\text { (degrees) }\end{array}$ & $\begin{array}{c}\quad \text { Plot } \\
\text { gradient } \\
\text { (degrees) }\end{array}$ & $\begin{array}{c}\text { Plot } \\
\text { veg- } \\
\text { etation } \\
\text { cover } \\
\text { (percent) }\end{array}$ & $\begin{array}{c}\text { Sim. } \\
\text { rainfall } \\
\text { duration } \\
\text { (min) }\end{array}$ & $\begin{array}{c}\text { Sim. } \\
\text { total } \\
\text { rainfall } \\
\text { depth } \\
(\mathrm{mm}) \\
\end{array}$ & $\begin{array}{c}\text { Sim. } \\
\text { mean } \\
\text { rainfall } \\
\text { rate } \\
(\mathrm{mm} / \mathrm{hr}) \\
\end{array}$ & $\begin{array}{c}\text { Sim. } \\
\text { total } \\
\text { rainfall } \\
\text { on plot } \\
(\mathrm{mL}) \\
\end{array}$ & $\begin{array}{c}\text { Sim. } \\
\text { total } \\
\text { runoff } \\
\text { from plot }{ }^{1} \\
(\mathrm{~mL}) \\
\end{array}$ & $\begin{array}{c}\text { Total } \\
\text { runoff } \\
\text { rate } \\
\left(\mathrm{mL} / \mathrm{m}^{2} / \mathrm{hr}\right) \\
\end{array}$ & $\begin{array}{c}\text { Sim. } \\
\text { runoff } \\
\text { (percent) }\end{array}$ \\
\hline \multicolumn{14}{|c|}{ Undisturbed plots: } \\
\hline ES.9SE_U & 1 & 146 & 69.8 & 32.5 & 37.5 & 0 & 90 & 58.2 & 38.8 & 58,200 & 5,982 & 3,988 & 10.3 \\
\hline ES.9NW_U & 3 & 297 & 70.2 & 26.8 & 26.5 & 25 & 90 & 77.0 & 51.3 & 77,000 & 11,759 & 7,839 & 15.3 \\
\hline ES.9SW־U & 7 & 207 & 91.4 & 23.8 & 24.0 & 6 & 90 & 81.2 & 54.1 & 81,200 & 11,275 & 7,517 & 13.9 \\
\hline ES.38NE_U & 5 & 40 & 115.0 & 26.0 & 27.0 & 25 & 90 & 68.0 & 45.3 & 68,000 & 20,627 & 13,751 & 30.3 \\
\hline ES.40E_Ū1 & 9 & 103 & 33.4 & 28.3 & 29.3 & 3 & 90 & 68.0 & 45.3 & 68,000 & 600 & 400 & 0.9 \\
\hline ES.40NW̄_U & 23 & 328 & 9.0 & 26.5 & 34.0 & 10 & 70 & 86.8 & 74.4 & 86,800 & 1,274 & 1,092 & 1.5 \\
\hline CP.8S_U & 13 & 186 & 51.8 & 37.9 & 35.0 & 0 & 82 & 52.4 & 38.3 & 52,400 & 21,864 & 15,998 & 41.7 \\
\hline CP.8W_U & 15 & 251 & 64.0 & 36.2 & 36.5 & 1 & 90 & 89.4 & 59.6 & 89,400 & 1,497 & 998 & 1.7 \\
\hline CP.8NW̄_U & 20 & 322 & 76.2 & 28.2 & 28.2 & 7 & 90 & 103.4 & 68.9 & 103,400 & 9,188 & 6,125 & 8.9 \\
\hline CP.8N_U & 17 & 5 & 75.0 & 26.8 & 29.0 & 10 & 90 & 80.0 & 53.3 & 80,000 & 22,883 & 15,255 & 28.6 \\
\hline CS.24Ē_U & 19 & 86 & 39.3 & 20.2 & 21.8 & 25 & 90 & 91.2 & 60.8 & 91,200 & 925 & 617 & 1.0 \\
\hline ES.40E_U2 & 10 & 103 & 33.4 & 28.3 & 29.3 & 3 & 90 & 69.6 & 46.4 & 69,600 & 1,287 & 858 & 1.8 \\
\hline \multicolumn{4}{|c|}{ Mean (1-day simulations) } & 28.5 & 29.9 & & & & 53.7 & & & 6,689 & 14.0 \\
\hline \multicolumn{14}{|c|}{ Disturbed plots: } \\
\hline ES.9SE_D & 2 & 146 & 69.8 & 32.5 & 33.5 & 0 & 90 & 73.4 & 48.9 & 73,400 & 7,829 & 5,219 & 10.7 \\
\hline ES.9NW̄D D & 4 & 297 & 69.2 & 26.8 & 23.5 & 10 & 90 & 75.0 & 50.0 & 75,000 & 35,387 & 23,591 & 47.2 \\
\hline ES.9SW_D & 8 & 206 & 91.4 & 23.8 & 26.3 & 1 & 120 & 107.0 & 53.5 & 107,000 & 1,031 & 516 & 1.0 \\
\hline ES.38NE_D & 6 & 40 & 115.0 & 26.0 & 23.0 & 2 & 90 & 76.2 & 50.8 & 76,200 & 22,352 & 14,901 & 29.3 \\
\hline ES.40E $\bar{D} 1$ & 11 & 103 & 33.4 & 28.3 & 32.0 & 2 & 90 & 73.6 & 49.1 & 73,600 & 1,210 & 807 & 1.6 \\
\hline ES.40NW_D & 24 & 320 & 9.0 & 26.5 & 31.0 & 6 & 120 & 95.6 & 47.8 & 95,600 & 2,150 & 1,075 & 2.2 \\
\hline CP.8S_D ${ }^{-}$ & 14 & 186 & 51.8 & 37.9 & 35.0 & 0 & 85 & 80.8 & 57.0 & 80,750 & 21,738 & 15,344 & 26.9 \\
\hline CP.8W_D & 16 & 240 & 64.0 & 36.2 & 35.0 & 0 & 90 & 76.6 & 51.1 & 76,600 & 3,634 & 2,423 & 4.7 \\
\hline CP.8NW̄_D1 & 21 & 316 & 77.7 & 28.2 & 28.0 & 2 & 90 & 82.6 & 55.1 & 82,600 & 10,974 & 7,316 & 13.3 \\
\hline CP.8N_D & 18 & 6 & 75.0 & 26.8 & 26.8 & 2 & 90 & 70.6 & 47.1 & 70,600 & 2,489 & 1,659 & 3.5 \\
\hline ES.40E_D2 & 12 & 103 & 33.4 & 28.3 & 32.0 & 2 & 90 & 70.8 & 47.2 & 70,800 & 2,538 & 1,692 & 3.6 \\
\hline CP.8NW__D2 & 22 & 316 & 77.7 & 28.2 & 28.0 & 2 & 90 & 90.2 & 60.1 & 90,200 & 25,706 & 17,137 & 28.5 \\
\hline \multicolumn{4}{|c|}{ Mean (1-day simulations) } & 29.3 & 29.4 & & & & 51.0 & & & 7,285 & 14.1 \\
\hline
\end{tabular}


Table 3. Rainfall-runoff and ancillary data from Gunnison Gorge National Conservation Area, southwestern Colorado.—Continued

[Sim., simulation; no., number; concn., concentration; Normal., normalized; rel $100 \mathrm{yr}$, variable is normalized to the 100 -year rainfall rate of $38 \mathrm{~mm}$ per hour; \&, and; min, minute; $\mathrm{mm}$, millimeter, $\mathrm{mm} / \mathrm{h}$, millimeter per hour; $\mathrm{mL}$, milliliter; $\mathrm{mL} / \mathrm{m}^{2} / \mathrm{h}$, milliliter per square meter per hour; g/L, grams per liter; g, grams; $\mathrm{g} / \mathrm{m}^{2} / \mathrm{h}$; grams per square meter per hour; $\mathrm{mL} / \mathrm{h}$; milliliter per hour; na, not applicable or no data; Yellow highlighted fields are from second-day rainfall simulations]

\begin{tabular}{|c|c|c|c|c|c|c|c|c|c|c|c|}
\hline $\begin{array}{l}\text { Rainfall- } \\
\text { runoff } \\
\text { plot } \\
\text { identification } \\
\text { code } \\
\end{array}$ & $\begin{array}{c}\text { Mean } \\
\text { sedi- } \\
\text { ment } \\
\text { concn. } \\
(\mathrm{g} / \mathrm{L}) \\
\end{array}$ & $\begin{array}{c}\text { Sim. } \\
\text { total } \\
\text { sediment } \\
\text { from plot } \\
\text { (g) } \\
\end{array}$ & $\begin{array}{c}\text { Total } \\
\text { sediment } \\
\text { yield } \\
\left(\mathrm{g} / \mathrm{m}^{2} / \mathrm{hr}\right)\end{array}$ & $\begin{array}{l}\text { Normal. } \\
\text { rainfall } \\
\text { rate } \\
\text { rel } 100 \mathrm{yr}^{2} \\
(\mathrm{~mm} / \mathrm{hr}) \\
\end{array}$ & $\begin{array}{c}\text { Normal. } \\
\text { runoff } \\
\text { rate } \\
\text { rel 100yr } \\
\left(\mathrm{mL} / \mathrm{m}^{2} / \mathrm{hr}\right) \\
\end{array}$ & $\begin{array}{l}\text { Normal. } \\
\text { sediment } \\
\text { yield } \\
\text { rel } 100 \mathrm{yr} \\
\left(\mathrm{g} / \mathrm{m}^{2} / \mathrm{hr}\right) \\
\end{array}$ & $\begin{array}{l}\text { Sim. } \\
\text { infiltra- } \\
\text { tion } \\
\text { volume } \\
\text { (mL) } \\
\end{array}$ & $\begin{array}{c}\text { Sim. } \\
\text { infiltra- } \\
\text { tion } \\
\text { (percent) }\end{array}$ & $\begin{array}{l}\text { Infiltra- } \\
\text { tion } \\
\text { rate } \\
(\mathrm{mL} / \mathrm{hr}) \\
\end{array}$ & $\begin{array}{l}\text { Normal. } \\
\text { infiltrat. } \\
\text { rate } \\
\text { rel } 100 \mathrm{yr} \\
\left(\mathrm{mL} / \mathrm{m}^{2} / \mathrm{hr}\right) \\
\end{array}$ & $\begin{array}{c}\text { Mean } \\
\text { wetting } \\
\text { front } \\
\text { depth } \\
(\mathrm{mm}) \\
\end{array}$ \\
\hline
\end{tabular}

Undisturbed plots:

\begin{tabular}{|c|c|c|c|c|c|c|c|c|c|c|c|}
\hline ES.9SE_U & 43.74 & 263 & 176 & 1.02 & 3,906 & 172 & 52,218 & 89.7 & 34,812 & 34,094 & 190.0 \\
\hline ES.9NW_U & 26.93 & 317 & 211 & 1.35 & 5,803 & 157 & 65,241 & 84.7 & 43,494 & 32,197 & 111.3 \\
\hline ES.9SW_U & 5.97 & 68.7 & 45.8 & 1.42 & 5,276 & 32.1 & 69,925 & 86.1 & 46,617 & 32,724 & 240.0 \\
\hline ES.38NE_U & 55.46 & 1,147 & 764 & 1.19 & 11,527 & 641 & 47,373 & 69.7 & 31,582 & 26,473 & 200.0 \\
\hline ES.40E_U1 & 0.32 & 0.18 & 0.12 & 1.19 & 335 & 0.10 & 67,400 & 99.1 & 44,933 & 37,665 & na \\
\hline ES.40NW_U & 1.16 & 1.60 & 1.37 & 1.96 & 558 & 0.70 & 85,526 & 98.5 & 73,308 & 37,442 & 240.0 \\
\hline CP.8S_U & 50.96 & 1,126 & 824 & 1.01 & 15,856 & 817 & 30,536 & 58.3 & 22,343 & 22,144 & 52.5 \\
\hline CP.8W_U & 8.45 & 14.22 & 9.48 & 1.57 & 636 & 6.04 & 87,903 & 98.3 & 58,602 & 37,364 & 300.0 \\
\hline CP.8NW_U & 24.44 & 216 & 144 & 1.81 & 3,377 & 79.3 & 94,212 & 91.1 & 62,808 & 34,623 & 222.5 \\
\hline CP.8N_U & 43.97 & 985 & 656 & 1.40 & 10,869 & 468 & 57,117 & 71.4 & 38,078 & 27,131 & 210.0 \\
\hline CS.24E_U & 1.07 & 0.97 & 0.65 & 1.60 & 385 & 0.40 & 90,275 & 99.0 & 60,183 & 37,615 & 620.0 \\
\hline ES.40E_U2 & 2.36 & 4.34 & 2.89 & 1.22 & 703 & 2.37 & 68,313 & 98.2 & 45,542 & 37,297 & 345.0 \\
\hline $\begin{array}{l}\text { Mean (1-day } \\
\text { simulations) }\end{array}$ & 23.86 & & & 1.41 & 5,321 & 216 & & 86.0 & 46,978 & 32,679 & 238.6 \\
\hline \multicolumn{12}{|c|}{ Disturbed plots } \\
\hline ES.9SE_D & 12.40 & 97.2 & 64.8 & 1.29 & 4,053 & 50.3 & 65,571 & 89.3 & 43,714 & 33,947 & 155.0 \\
\hline ES.9NW_D & 27.87 & 999 & 666 & 1.32 & 17,929 & 506 & 39,613 & 52.8 & 26,409 & 20,071 & 105.0 \\
\hline ES.9SW_D & 0.81 & 0.88 & 0.44 & 1.41 & 366 & 0.31 & 105,969 & 99.0 & 52,985 & 37,634 & 270.0 \\
\hline ES.38NE_D & 67.52 & 1,513 & 1,008 & 1.34 & 11,147 & 754 & 53,848 & 70.7 & 35,899 & 26,853 & 201.3 \\
\hline ES.40E_D1 & 0.68 & 0.78 & 0.52 & 1.29 & 625 & 0.40 & 72,390 & 98.4 & 48,260 & 37,375 & na \\
\hline ES.40NW_D & 0.16 & 0.36 & 0.18 & 1.26 & 855 & 0.14 & 93,450 & 97.8 & 46,725 & 37,145 & 330.0 \\
\hline CP.8S_D & 75.90 & 1,644 & 1,161 & 1.50 & 10,230 & 774 & 59,012 & 73.1 & 41,656 & 27,770 & 70.0 \\
\hline CP.8W_D & 1.88 & 7.97 & 5.31 & 1.34 & 1,803 & 3.95 & 72,966 & 95.3 & 48,644 & 36,197 & 195.0 \\
\hline CP.8NW_D1 & 16.41 & 161 & 108 & 1.45 & 5,049 & 74.3 & 71,626 & 86.7 & 47,751 & 32,951 & na \\
\hline CP.8N_D & 4.75 & 12.7 & 8.45 & 1.24 & 1,340 & 6.82 & 68,111 & 96.5 & 45,407 & 36,660 & 191.3 \\
\hline ES.40E_D2 & 0.97 & 2.47 & 1.65 & 1.24 & 1,362 & 1.33 & 68,262 & 96.4 & 45,508 & 36,638 & 460.0 \\
\hline CP.8NW_D2 & 6.53 & 164 & 109 & 1.58 & 10,830 & 69.1 & 64,494 & 71.5 & 42,996 & 27,170 & na \\
\hline $\begin{array}{l}\text { Mean (1-day } \\
\text { simulations) }\end{array}$ & 20.84 & & & 1.34 & 5,340 & 217 & & 85.9 & 43,745 & 32,660 & 189.7 \\
\hline
\end{tabular}


Table 3. Rainfall-runoff and ancillary data from Gunnison Gorge National Conservation Area, southwestern Colorado.—Continued

[Sim., simulation; no., number; concn., concentration; Normal., normalized; rel $100 \mathrm{yr}$, variable is normalized to the 100-year rainfall rate of $38 \mathrm{~mm}$ per hour; \&, and; min, minute; mm, millimeter, mm/h, millimeter per hour; $\mathrm{mL}$, milliliter; $\mathrm{mL} / \mathrm{m}^{2} / \mathrm{h}$, milliliter per square meter per hour; g/L, grams per liter; g, grams; g/m²/h; grams per square meter per hour; $\mathrm{mL} / \mathrm{h} ;$ milliliter per hour; na, not applicable or no data; Yellow highlighted fields are from second-day rainfall simulations]

\begin{tabular}{|c|c|c|c|c|c|c|c|c|c|}
\hline $\begin{array}{c}\text { Rainfall- } \\
\text { runoff } \\
\text { plot } \\
\text { identification } \\
\text { code } \\
\end{array}$ & $\begin{array}{l}\text { Early sim. } \\
\text { runoff } \\
\text { silt } \& \text { clay } \\
\text { content }^{3} \\
\text { (percent) } \\
\end{array}$ & $\begin{array}{l}\text { Mid. sim. } \\
\text { runoff } \\
\text { silt \& clay } \\
\text { content }^{3} \\
\text { (percent) } \\
\end{array}$ & $\begin{array}{l}\text { Late sim. } \\
\text { runoff } \\
\text { silt \& clay } \\
\text { content }^{3} \\
\text { (percent) }\end{array}$ & $\begin{array}{c}\text { Mean } \\
\text { runoff } \\
\text { silt \& clay } \\
\text { content } \\
\text { (percent) } \\
\end{array}$ & $\begin{array}{c}\text { Plot } \\
\text { crust } \\
\text { silt \& clay } \\
\text { content } \\
\text { (percent) } \\
\end{array}$ & $\begin{array}{c}\text { Plot } \\
\text { crust } \\
\text { median } \\
\text { size } \\
(\mathrm{mm}) \\
\end{array}$ & $\begin{array}{c}\text { Plot soil color, } \\
\text { Munsell color } \\
\text { notation, } \\
\text { larger area } \\
\text { [smaller area] }\end{array}$ & $\begin{array}{c}\text { Plot soil color, } \\
\text { Munsell color } \\
\text { name, } \\
\text { larger area }^{4}\end{array}$ & Remark \\
\hline \multicolumn{10}{|l|}{ Undisturbed plots: } \\
\hline ES.9SE_U & 92.1 & 92.9 & 85.6 & 90.2 & 93.8 & 0.0056 & 10YR $8 / 2$ & Very pale brown & \\
\hline ES.9NW_U & 96.6 & 97.1 & 70.9 & 88.2 & 92.6 & 0.0041 & 10YR 7.5/2 [10YR 6/2] & Very pale brown & \\
\hline ES.9SW_U & 84.2 & 88.4 & 84.2 & 85.6 & 92.4 & 0.0046 & 10YR 8/2 [10YR 7/3] & Very pale brown & \\
\hline ES.38NE_U U & 97.5 & 96.4 & 95.9 & 96.6 & 92.4 & 0.0057 & $7.5 \mathrm{YR} 7 / 2$ & Pinkish gray & \\
\hline ES.40E_Ū1 & na & na & na & na & 87.7 & 0.0097 & 10YR 7/2 [7.5YR 8/1] & Light gray & Not excavated \\
\hline ES.40NW_U & 93.3 & 100.0 & 72.2 & 88.5 & 91.8 & 0.0060 & 7.5YR $7 / 2$ [7.5YR 7/1] & Pinkish gray & 70-minute simulation \\
\hline CP.8S_U ${ }^{-}$ & 86.4 & 86.2 & 76.9 & 83.2 & 94.3 & 0.0053 & 7.5YR $7 / 1$ & Light gray & 82-minute simulation \\
\hline CP.8W_U & 76.9 & 80.3 & 83.9 & 80.3 & 94.3 & 0.0052 & 10YR $7.5 / 3$ & Very pale brown & \\
\hline CP.8NW̄_u & 96.4 & 95.2 & 96.6 & 96.1 & 88.8 & 0.0070 & 10YR 8/2 [10YR 7/2] & Very pale brown & \\
\hline CP.8N_U & 95.8 & 93.4 & 94.1 & 94.4 & 89.9 & 0.0035 & 10YR $8 / 2$ & Very pale brown & \\
\hline CS.24E_U & 92.3 & 91.7 & 92.3 & 92.1 & 72.8 & 0.0144 & na & na & \\
\hline ES.40E_U2 & na & na & na & 99.2 & na & na & na & na & Second-day simulation \\
\hline $\begin{array}{l}\text { Mean (1-day } \\
\text { simulations) }\end{array}$ & 91.1 & 92.2 & 85.3 & 89.5 & 90.1 & 0.0065 & & & Means exclude second day sample \\
\hline \multicolumn{10}{|l|}{ Disturbed plots } \\
\hline ES.9SE_D & 98.3 & 95.7 & 93.1 & 95.7 & 95.4 & 0.0050 & 10YR 6/2 [10YR 7.5/2] & Light brownish gray & \\
\hline ES.9NW_D & 97.0 & 97.1 & 97.0 & 97.0 & 85.4 & 0.0095 & 10YR $8 / 2$ & Very pale brown & \\
\hline ES.9SW_D & na & na & na & na & 92.2 & 0.0057 & 10YR $7 / 3$ & Very pale brown & 120-minute simulation \\
\hline ES.38NE_D & 93.3 & 93.1 & 94.5 & 93.6 & 93.2 & 0.0056 & $7.5 y r 7 / 1$ & Light gray & \\
\hline ES.40E_D1 & na & na & na & na & 93.5 & 0.0065 & 10YR 7/2 & Light gray & Not excavated \\
\hline ES.40NW_D & 100.0 & 100.0 & 100.0 & 100.0 & 90.5 & 0.0060 & 7.5YR 7/2 & Pinkish gray & 120-minute simulation \\
\hline CP.8S_D & 92.2 & 90.3 & 91.6 & 91.4 & 95.7 & 0.0050 & 10YR 7/2 & Light gray & 85-minute simulation \\
\hline CP.8W_D & na & na & na & 93.4 & 95.8 & 0.0051 & 10YR $7.5 / 3$ & Very pale brown & \\
\hline CP.8NW̄_D1 & 92.8 & 90.4 & 93.0 & 92.1 & 85.5 & 0.0084 & 10YR $7 / 2$ & Light gray & Not excavted \\
\hline CP.8N_D- & na & na & na & 94.1 & 82.6 & 0.0084 & 10YR 7/2 & Light gray & \\
\hline ES.40E_D2 & na & na & na & na & na & na & na & na & Second-day simulation \\
\hline CP.8NW__D2 & 89.1 & 92.6 & 93.6 & 91.8 & 86.9 & 0.0078 & na & na & Second-day simulation \\
\hline $\begin{array}{l}\text { Mean (1-day } \\
\text { simulations) }\end{array}$ & 95.6 & 94.4 & 94.9 & 94.7 & 91.0 & 0.0065 & & & Means exclude second day sample \\
\hline
\end{tabular}

${ }^{1}$ Simulation total rainfall on plot $=1 \mathrm{~m}^{2} \times$ duration of rainfall, with $1 \mathrm{~mL}=\mathrm{cm}^{3}$, or rainfall $(\mathrm{mm}) \times 1,000=\mathrm{mL}$.

${ }^{2}$ Normalized rainfall $=60$ minutes mean rainfall $/ 38 \mathrm{~mm} 100$-yr design storm.

${ }^{3}$ Early, mid-, and late samples for percent silt and clay taken at 25, 50, and 75 percent of the runoff hydrograph duration.

${ }^{4}$ Soil colors are for dry soil onsite (Munsell Color, 1992). 
Table 4. Rainfall-runoff and ancillary data from Gunnison Gorge National Conservation Area, southwestern Colorado.

Sim., simulation; no., number; trav, microtopography traverse; mm, millimeter, mm/h, millimeter per hour; $\mathrm{mL}$, milliliter; $\mathrm{mL} / \mathrm{m}^{2} / \mathrm{h}$, milliliter per square meter per hour; g, grams; g/m²/h; grams per square meter per hour; $\mathrm{mL} / \mathrm{h}$; milliliter per hour; na, not appplicable or no data]

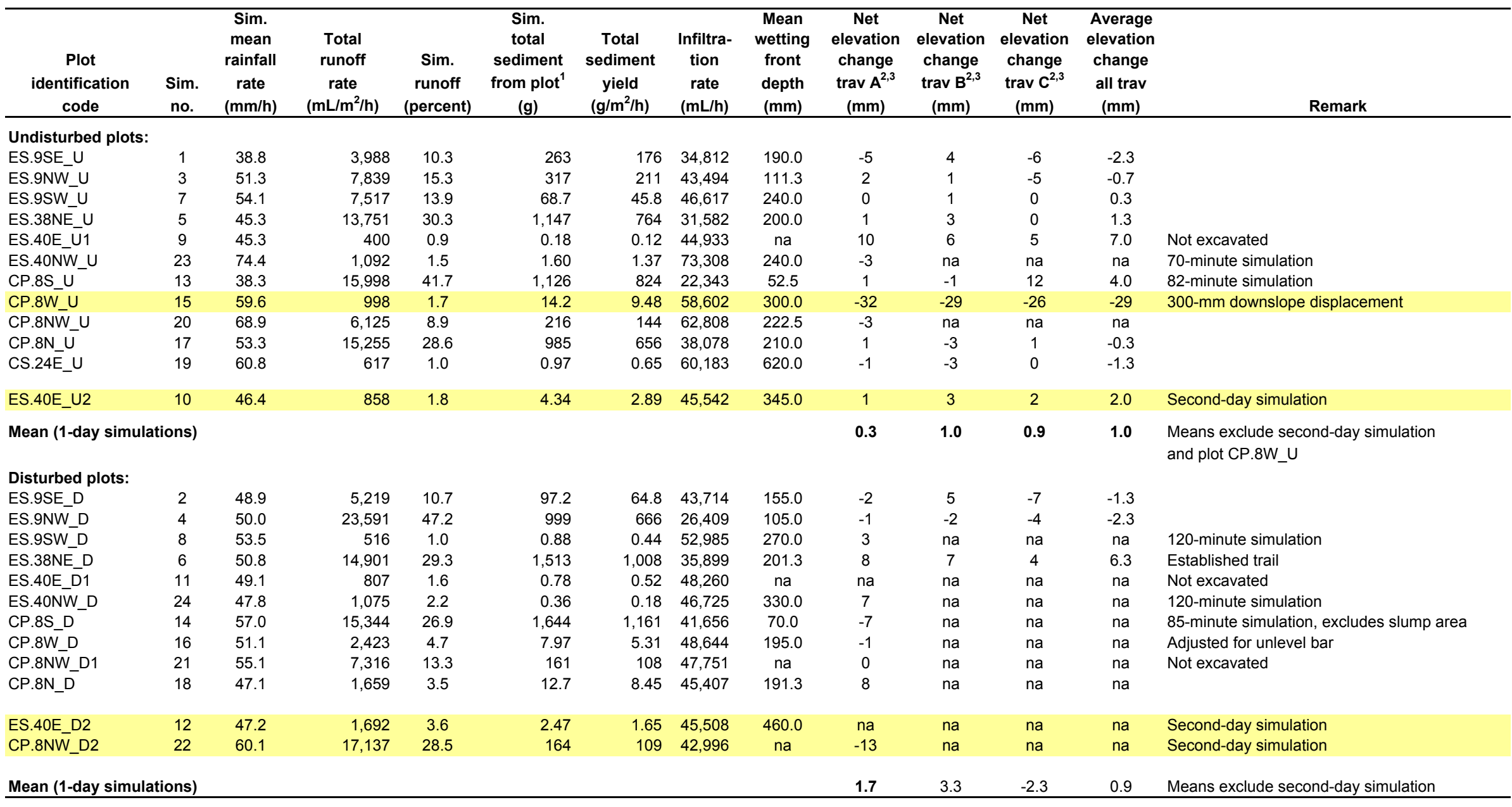

${ }^{1}$ Simulation total rainfall on plot $=1 \mathrm{~m}^{2} \times$ duration of rainfall, with $1 \mathrm{~mL}=1 \mathrm{~cm}^{3}$, or rainfall $(\mathrm{mm}) \times 1,000=\mathrm{mL}$.

${ }^{2}$ Traverse A located $250 \mathrm{~mm}$ from plot upper boundary; Traverse B located $500 \mathrm{~mm}$ from plot upper boundary; Traverse C located $750 \mathrm{~mm}$ from plot upper boundary.

${ }^{3}$ Each microtopographic traverse consisted of 49 point-elevation measurements. 
Table 5. Hillslope-creep rates and ancillary data from Gunnison Gorge National Conservation Area, southwestern Colorado.

[Creep monitors are wired flags inserted upslope from rainfall-runoff simulation plot microtopographic reference stakes; mm, millimeters; mm/d, millimeters per day; $\mathrm{mm} / \mathrm{yr}$, millimeters per year; , approximately; na, not applicable or no data]

\begin{tabular}{|c|c|c|c|c|c|c|c|c|c|c|c|c|}
\hline \multirow{3}{*}{$\begin{array}{l}\text { Rainfall-runoff plot } \\
\text { identification code }\end{array}$} & \multirow{3}{*}{$\begin{array}{l}\text { Date creep } \\
\text { flags installed }\end{array}$} & \multirow{3}{*}{$\begin{array}{c}\text { Julian } \\
\text { day since } \\
\text { January } \\
2003\end{array}$} & \multirow{3}{*}{$\begin{array}{c}\begin{array}{c}\text { Surveyed } \\
\text { hillslope } \\
\text { aspect }\end{array} \\
\text { (degrees) }\end{array}$} & \multirow[b]{2}{*}{$\begin{array}{l}\text { Hillslope } \\
\text { length } \\
\text { upslope from } \\
\text { rainfall plot, } \\
\text { approximate }\end{array}$} & \multirow[b]{2}{*}{$\begin{array}{l}\text { Surveyed } \\
\text { hillslope } \\
\text { gradient }\end{array}$} & \multirow{2}{*}{\begin{tabular}{c|} 
\\
Rainfall \\
plot \\
gradient
\end{tabular}} & \multicolumn{6}{|c|}{\begin{tabular}{|l|} 
Late summer, fall and winter $2003 / 2004$ \\
(since August 2003, $\sim 8$ months)
\end{tabular}} \\
\hline & & & & & & & $\begin{array}{c}\text { Date } \\
\text { observed }\end{array}$ & $\begin{array}{c}\text { Julian } \\
\text { day } \\
\text { since } \\
\text { January } \\
2003\end{array}$ & $\begin{array}{l}\text { Elapsed } \\
\text { number } \\
\text { of days }\end{array}$ & $\begin{array}{c}\text { Mean } \\
\text { downslope } \\
\text { creep } \\
\text { distance }\end{array}$ & $\begin{array}{c}\text { Mean } \\
\text { creep } \\
\text { rate }\end{array}$ & $\begin{array}{l}\text { Seasonal } \\
\text { creep rate }\end{array}$ \\
\hline & & & & (meters) & (degrees) & (degrees) & & & & $(\mathrm{mm})$ & $(\mathrm{mm} / \mathrm{d})$ & $(\mathrm{mm} / \mathrm{yr})$ \\
\hline \multicolumn{13}{|l|}{ Undisturbed plots: } \\
\hline ES.9SE_U & 13 Aug 03 & 225 & 146 & 69.8 & 32.6 & 37.5 & 15 Apr 04 & 471 & 246 & 70.8 & 0.288 & 105 \\
\hline ES.9NW_U & 14 Aug 03 & 226 & 297 & 70.2 & 26.8 & 26.5 & 15 Apr 04 & 471 & 245 & 72.2 & 0.295 & 108 \\
\hline ES.9SW_U & 15 Apr 04 & 471 & 207 & 91.4 & 23.8 & 24.0 & 15 Apr 04 & 471 & 0 & na & na & na \\
\hline ES.38NE_U & 20 Aug 03 & 232 & 40 & 115.0 & 26.0 & 27.0 & 15 Apr 04 & 471 & 239 & -9.00 & -0.038 & -13.7 \\
\hline ES.40E_Ū & 4 Aug 04 & 582 & 103 & 33.4 & 28.3 & 29.3 & & & & & & \\
\hline ES.40NW_U & 8 Jun 05 & 890 & 328 & 9.0 & 26.5 & 34.0 & & & & & & \\
\hline CP.8S_U & 4 May 04 & 490 & 186 & 51.8 & 37.9 & 35.0 & & & & & & \\
\hline CP.8W_U & 5 May 04 & 491 & 251 & 64.0 & 36.2 & 36.5 & & & & & & \\
\hline CP.8NW̄_U & 3 May 05 & 854 & 322 & 76.2 & 28.2 & 28.2 & & & & & & \\
\hline CP.8N_Ū & 6 May 04 & 492 & 5 & 75.0 & 26.8 & 29.0 & & & & & & \\
\hline CS.24E_U & 7 May 04 & 493 & 86 & 39.3 & 20.2 & 21.8 & & & & & & \\
\hline Mean undisturbed & & & & & 28.5 & 29.9 & & & & & & 66.3 \\
\hline \multicolumn{13}{|l|}{ Disturbed plots: } \\
\hline ES.9SE_D & 19 Aug 03 & 231 & 146 & 69.8 & 32.5 & 33.5 & 15 Apr 04 & 471 & 240 & 62.2 & 0.259 & 94.6 \\
\hline ES.9NW_D_D & 22 Aug 03 & 234 & 297 & 69.2 & 26.8 & 23.5 & 15 Apr 04 & 471 & 237 & 38.7 & 0.163 & 59.6 \\
\hline ES.9SW_D & 13 Aug 04 & 591 & 206 & 91.4 & 23.8 & 26.3 & & & & & & \\
\hline ES.38NE_D & none & na & 40 & 115.0 & 26.0 & 23.0 & na & na & na & na & na & na \\
\hline ES.40E_D & 6 Aug 04 & 584 & 103 & 33.4 & 28.3 & 32.0 & & & & & & \\
\hline ES.40NW_D & 9 Jun 05 & 891 & 320 & 9.0 & 26.5 & 31.0 & & & & & & \\
\hline CP.8S_D & 10 Aug 04 & 588 & 186 & 51.8 & 37.9 & 35.0 & & & & & & \\
\hline CP.8W_D & 11 Aug 04 & 589 & 240 & 64.0 & 36.2 & 35.0 & & & & & & \\
\hline CP.8NW̄_D & 4 May 05 & 855 & 316 & 77.7 & 28.2 & 28.0 & & & & & & \\
\hline CP.8N_D & 12 Aug 04 & 590 & 6 & 75.0 & 26.8 & 26.8 & & & & & & \\
\hline Mean disturbed & & & & & 29.3 & 29.4 & & & & & & 77.1 \\
\hline
\end{tabular}


Table 5. Hillslope-creep rates and ancillary data from Gunnison Gorge National Conservation Area, southwestern Colorado.-Continued

[Creep monitors are wired flags inserted upslope from rainfall-runoff simulation plot microtopographic reference stakes; mm, millimeters; mm/d, millimeters per day; $\mathrm{mm} / \mathrm{yr}$, millimeters per year; , approximately; na, not applicable or no data]

\begin{tabular}{|c|c|c|c|c|c|c|c|c|c|c|c|c|c|}
\hline \multirow[b]{2}{*}{$\begin{array}{l}\text { Rainfall-runoff plot } \\
\text { identification code }\end{array}$} & \multirow{2}{*}{$\begin{array}{c}\text { Date creep } \\
\text { flags installed }\end{array}$} & \multicolumn{6}{|c|}{$\begin{array}{l}\text { Full year April } 2004 \text { - April } 2005 \\
\text { (since April 2004 12 months, since May } 2004 \sim 11 \text { months) }\end{array}$} & \multicolumn{6}{|c|}{$\begin{array}{l}\text { Late summer, fall and winter } 2004 \text { / } 2005 \\
\text { (since August 2004, } \sim 8 \text { months) }\end{array}$} \\
\hline & & \begin{tabular}{|c|} 
Date \\
observed \\
or \\
installed
\end{tabular} & $\begin{array}{c}\text { Julian } \\
\text { day } \\
\text { since } \\
\text { January } \\
2003\end{array}$ & $\begin{array}{l}\text { Elapsed } \\
\text { number } \\
\text { of days }\end{array}$ & $\begin{array}{c}\text { Mean } \\
\text { downslope } \\
\text { creep } \\
\text { distance }\end{array}$ & $\begin{array}{c}\text { Mean } \\
\text { creep } \\
\text { rate }\end{array}$ & $\begin{array}{c}\text { Annual } \\
\text { creep } \\
\text { rate }\end{array}$ & $\begin{array}{c}\text { Date } \\
\text { observed }\end{array}$ & $\begin{array}{c}\text { Julian } \\
\text { day since } \\
\text { January } \\
2003\end{array}$ & $\begin{array}{l}\text { Elapsed } \\
\text { number } \\
\text { of days }\end{array}$ & $\begin{array}{c}\text { Mean } \\
\text { downslope } \\
\text { creep } \\
\text { distance } \\
\\
(\mathrm{mm}) \\
\end{array}$ & $(\mathrm{mm} / \mathrm{d})$ & $\begin{array}{c}\text { Seasonal } \\
\text { creep rate } \\
(\mathrm{mm} / \mathrm{yr})\end{array}$ \\
\hline \multicolumn{14}{|l|}{ Undisturbed plots: } \\
\hline ES.9SE_U & 13 Aug 03 & 27 Apr 05 & 848 & 377 & 12.8 & 0.034 & 12.4 & & & & & & \\
\hline ES.9NW_U & 14 Aug 03 & 27 Apr 05 & 848 & 377 & 10.2 & 0.027 & 9.88 & & & & & & \\
\hline ES.9SW_U & 15 Apr 04 & 27 Apr 05 & 848 & 377 & na & na & na & & & & & & \\
\hline ES.38NE_U & 20 Aug 03 & 27 Apr 05 & 848 & 377 & -2.00 & -0.005 & -1.94 & & & & & & \\
\hline ES.40E_Ū & 4 Aug 04 & & & & & & & 27 Apr 05 & 848 & 266 & 38.8 & 0.146 & 53.2 \\
\hline ES.40NW_U & 8 Jun 05 & 8 Jun 05 & 890 & 0 & na & na & na & & & & & & \\
\hline CP.8S_U & 4 May 04 & 9 Apr 05 & 830 & 340 & 85.2 & 0.251 & 91.5 & & & & & & \\
\hline CP.8W_U & 5 May 04 & 9 Apr 05 & 830 & 339 & 95.5 & 0.282 & 103 & & & & & & \\
\hline CP.8NW_U & 3 May 05 & 3 May 05 & 854 & 0 & na & na & na & & & & & & \\
\hline CP.8N_U & 6 May 04 & 3 Apr 05 & 824 & 332 & 0.0 & 0.000 & 0.000 & & & & & & \\
\hline CS.24E-U & 7 May 04 & 9 Apr 05 & 830 & 337 & 40.5 & 0.120 & 43.9 & & & & & & \\
\hline Mean undisturbed & & & & & & & 36.9 & & & & & & 53.2 \\
\hline \multicolumn{14}{|l|}{ Disturbed plots: } \\
\hline ES.9SE_D & 19 Aug 03 & 27 Apr 05 & 848 & 377 & 19.0 & 0.050 & 18.4 & & & & & & \\
\hline ES.9NW_D & 22 Aug 03 & 27 Apr 05 & 848 & 377 & 3.8 & 0.010 & 3.68 & & & & & & \\
\hline ES.9SW_D & 13 Aug 04 & & & & & & & 27 Apr 05 & 848 & 257 & 39.2 & 0.153 & 55.7 \\
\hline ES.38NE_D & none & na & na & na & na & na & na & na & na & na & na & na & na \\
\hline ES.40E_D & 6 Aug 04 & & & & & & & $27 \mathrm{Apr} 05$ & 848 & 264 & 18.3 & 0.0693 & 25.3 \\
\hline ES.40NW_D & 9 Jun 05 & 9 Jun 05 & 891 & 0 & na & na & na & & & & & & \\
\hline CP.8S_D & 10 Aug 04 & & & & & & & 9 Apr 05 & 830 & 242 & 105 & 0.432 & 158 \\
\hline CP.8W_D & 11 Aug 04 & & & & & & & 9 Apr 05 & 830 & 241 & 108 & 0.449 & 164 \\
\hline CP.8NW_D & 4 May 05 & 4 May 05 & 855 & 0 & na & na & na & & & & & & \\
\hline CP.8N_D & 12 Aug 04 & & & & & & & 3 Apr 05 & 824 & 234 & 21.2 & 0.091 & 33.1 \\
\hline Mean disturbed & & & & & & & 11.0 & & & & & & 87.2 \\
\hline
\end{tabular}


Table 5. Hillslope-creep rates and ancillary data from Gunnison Gorge National Conservation Area, southwestern Colorado.-Continued

[Creep monitors are wired flags inserted upslope from rainfall-runoff simulation plot microtopographic reference stakes; mm, millimeters; mm/d, millimeters per day; mm/yr, millimeters per year; , approximately; na, not applicable or no data]

\begin{tabular}{|c|c|c|c|c|c|c|c|c|c|c|c|c|c|}
\hline \multirow{3}{*}{$\begin{array}{l}\text { Rainfall-runoff plot } \\
\text { identification code }\end{array}$} & \multirow{3}{*}{$\begin{array}{c}\text { Date creep } \\
\text { flags installed }\end{array}$} & \multicolumn{6}{|c|}{$\begin{array}{l}\text { Spring and summer } 2005 \\
\text { (since April and May } 2005 \sim 6 \text { months, or June } \sim 4.6 \text { months) }\end{array}$} & \multicolumn{6}{|c|}{$\begin{array}{l}\text { Fall and winter } 2005 \text { / } 2006 \\
\text { (since October or November } 2005 \sim 5.5 \text { to } 6 \text { months) }\end{array}$} \\
\hline & & $\begin{array}{c}\text { Date } \\
\text { observed }\end{array}$ & $\begin{array}{c}\text { Julian } \\
\text { day } \\
\text { since } \\
\text { January } \\
2003\end{array}$ & $\begin{array}{c}\text { Elapsed } \\
\text { number } \\
\text { of days }\end{array}$ & $\begin{array}{c}\text { Mean } \\
\text { downslope } \\
\text { creep } \\
\text { distance }\end{array}$ & $\begin{array}{c}\text { Mean } \\
\text { creep rate }\end{array}$ & $\begin{array}{l}\text { Seasonal } \\
\text { creep rate }\end{array}$ & $\begin{array}{c}\text { Date } \\
\text { observed }\end{array}$ & $\begin{array}{c}\text { Julian } \\
\text { day } \\
\text { since } \\
\text { January } \\
2003\end{array}$ & $\begin{array}{c}\text { Elapsed } \\
\text { number } \\
\text { of days }\end{array}$ & $\begin{array}{c}\text { Mean } \\
\text { downslope } \\
\text { creep } \\
\text { distance }\end{array}$ & $\begin{array}{c}\text { Mean } \\
\text { creep rate }\end{array}$ & $\begin{array}{l}\text { Seasonal } \\
\text { creep rate }\end{array}$ \\
\hline & & & & & $(\mathrm{mm})$ & $(\mathrm{mm} / \mathrm{d})$ & $(\mathrm{mm} / \mathrm{yr})$ & & & & $(\mathrm{mm})$ & $(\mathrm{mm} / \mathrm{d})$ & $(\mathrm{mm} / \mathrm{yr})$ \\
\hline \multicolumn{14}{|l|}{ Undisturbed plots: } \\
\hline ES.9SE_U & 13 Aug 03 & 25 Oct 05 & 1,029 & 181 & 8.00 & 0.0442 & 16.1 & 26 Apr 06 & 1212 & 183 & 3.70 & 0.0202 & 7.38 \\
\hline ES.9NW_U & 14 Aug 03 & 25 Oct 05 & 1,029 & 181 & 7.00 & 0.0387 & 14.1 & 26 Apr 06 & 1212 & 183 & 3.50 & 0.0191 & 6.98 \\
\hline ES.9SW_U & 15 Apr 04 & 25 Oct 05 & 1,029 & 181 & 13.2 & 0.0729 & 26.6 & 26 Apr 06 & 1212 & 183 & 2.00 & 0.0109 & 3.99 \\
\hline ES.38NE_U & 20 Aug 03 & 25 Oct 05 & 1,029 & 181 & 12.7 & 0.0702 & 25.6 & 26 Apr 06 & 1212 & 183 & 0.700 & 0.00383 & 1.40 \\
\hline ES.40E_U & 4 Aug 04 & 25 Oct 05 & 1,029 & 181 & 12.0 & 0.0663 & 24.2 & 26 Apr 06 & 1212 & 183 & 5.40 & 0.0295 & 10.8 \\
\hline ES.40NW_U & 8 Jun 05 & 25 Oct 05 & 1,029 & 139 & 15.3 & 0.110 & 40.2 & 26 Apr 06 & 1212 & 183 & 0.50 & 0.00273 & 0.997 \\
\hline CP.8S_U & 4 May 04 & 10 Nov 05 & 1,045 & 215 & 4.30 & 0.0200 & 7.30 & 24 Apr 06 & 1210 & 165 & 1.50 & 0.00909 & 3.32 \\
\hline CP.8W_U & 5 May 04 & 10 Nov 05 & 1,045 & 215 & 3.30 & 0.0153 & 5.60 & 24 Apr 06 & 1210 & 165 & 2.30 & 0.0139 & 5.09 \\
\hline CP.8NW_U & 3 May 05 & 10 Nov 05 & 1,045 & 191 & 7.80 & 0.0408 & 14.9 & 24 Apr 06 & 1210 & 165 & -1.30 & -0.00788 & -2.88 \\
\hline CP.8N_U & 6 May 04 & 10 Nov 05 & 1,045 & 221 & 7.70 & 0.0348 & 12.7 & 24 Apr 06 & 1210 & 165 & -0.500 & -0.00303 & -1.11 \\
\hline CS.24E_U & 7 May 04 & 10 Nov 05 & 1,045 & 215 & 5.50 & 0.0256 & 9.34 & 24 Apr 06 & 1210 & 165 & 1.20 & 0.00727 & 2.65 \\
\hline Mean undisturbed & & & & & & & 17.9 & & & & & & 3.51 \\
\hline \multicolumn{14}{|l|}{ Disturbed plots: } \\
\hline ES.9SE_D & 19 Aug 03 & 25 Oct 05 & 1,029 & 181 & 8.70 & 0.048 & 17.5 & 26 Apr 06 & 1212 & 183 & 4.00 & 0.0219 & 7.98 \\
\hline ES.9NW_D & 22 Aug 03 & 25 Oct 05 & 1,029 & 181 & 3.00 & 0.017 & 6.05 & 26 Apr 06 & 1212 & 183 & 2.70 & 0.0148 & 5.39 \\
\hline ES.9SW_D & 13 Aug 04 & 25 Oct 05 & 1,029 & 181 & 0.500 & 0.003 & 1.01 & 26 Apr 06 & 1212 & 183 & -1.20 & -0.00656 & -2.39 \\
\hline ES.38NE_D & none & na & na & na & na & na & na & na & na & na & na & na & na \\
\hline ES.40E_D & 6 Aug 04 & 25 Oct 05 & 1,029 & 181 & 3.20 & 0.018 & 6.45 & 26 Apr 06 & 1212 & 183 & 0.200 & 0.00109 & 0.399 \\
\hline ES.40NW_D & 9 Jun 05 & 25 Oct 05 & 1,029 & 138 & 12.2 & 0.088 & 32.3 & 26 Apr 06 & 1212 & 183 & 1.00 & 0.00546 & 1.99 \\
\hline CP.8S_D & 10 Aug 04 & 10 Nov 05 & 1,045 & 215 & 3.80 & 0.018 & 6.45 & 24 Apr 06 & 1210 & 165 & 0.400 & 0.00242 & 0.885 \\
\hline CP.8W_D & 11 Aug 04 & 10 Nov 05 & 1,045 & 215 & 4.50 & 0.021 & 7.64 & 24 Apr 06 & 1210 & 165 & -1.80 & -0.0109 & -3.98 \\
\hline CP.8NW_D & 4 May 05 & 10 Nov 05 & 1,045 & 190 & na & na & na & 24 Apr 06 & 1210 & 165 & na & na & na \\
\hline CP.8N_D & 12 Aug 04 & 10 Nov 05 & 1,045 & 221 & -0.200 & -0.001 & -0.330 & 24 Apr 06 & 1210 & 165 & -2.20 & -0.0133 & -4.87 \\
\hline Mean disturbed & & & & & & & 9.6 & & & & & & 0.675 \\
\hline
\end{tabular}


Table 5. Hillslope-creep rates and ancillary data from Gunnison Gorge National Conservation Area, southwestern Colorado.-Continued

[Creep monitors are wired flags inserted upslope from rainfall-runoff simulation plot microtopographic reference stakes; mm, millimeters; mm/d, millimeters per day; mm/yr, millimeters per year; , approximately; na, not applicable or no data]

\begin{tabular}{|c|c|c|c|c|c|c|c|c|}
\hline \multirow[b]{2}{*}{$\begin{array}{l}\text { Rainfall-runoff plot } \\
\text { identification code }\end{array}$} & \multirow[b]{2}{*}{$\begin{array}{c}\text { Date creep } \\
\text { flags installed }\end{array}$} & \multicolumn{6}{|c|}{\begin{tabular}{|l|} 
Full year April 2005 - April 2006 \\
(since April $2005 \sim 12$ months) \\
\end{tabular}} & \multirow[b]{2}{*}{ Remark } \\
\hline & & $\begin{array}{c}\text { Date } \\
\text { observed }\end{array}$ & $\begin{array}{l}\text { Julian day } \\
\text { since } \\
\text { January } \\
2003\end{array}$ & $\begin{array}{l}\text { Elapsed } \\
\text { number } \\
\text { of days }\end{array}$ & $\begin{array}{l}\text { Mean } \\
\text { downslope } \\
\text { creep } \\
\text { distance } \\
\\
(\mathrm{mm}) \\
\end{array}$ & $\begin{array}{l}\text { Mean } \\
\text { creep rate } \\
(\mathrm{mm} / \mathrm{d})\end{array}$ & $\begin{array}{l}\text { Annual } \\
\text { creep } \\
\text { rate } \\
\end{array}$ & \\
\hline \multicolumn{8}{|l|}{ Undisturbed plots: } & \multirow{13}{*}{$\begin{array}{l}\text { Bar in different location before April 2005. Five wires bent by } 25 \text { October } 2005 . \\
\text { Only two flags measured } 15 \text { April } 2004 \text {. R250 reference pin reestablished } 27 \text { April } 2005 . \\
\text { Number } 4 \text { wire out by } 27 \text { April } 2005 .\end{array}$} \\
\hline ES.9SE_U & 13 Aug 03 & 26 Apr 06 & 1212 & 364 & 11.7 & 0.0321 & 11.7 & \\
\hline ES.9NW_U & 14 Aug 03 & 26 Apr 06 & 1212 & 364 & 10.5 & 0.0288 & 10.5 & \\
\hline ES.9SW_U & 15 Apr 04 & 26 Apr 06 & 1212 & 364 & 15.2 & 0.0418 & 15.2 & \\
\hline ES.38NE_U & 20 Aug 03 & 26 Apr 06 & 1212 & 364 & 13.3 & 0.0365 & 13.3 & \\
\hline ES.40E_U & 4 Aug 04 & 26 Apr 06 & 1212 & 364 & 17.4 & 0.0478 & 17.4 & \\
\hline ES.40NW_U & 8 Jun 05 & 26 Apr 06 & 1212 & 322 & 15.8 & 0.0491 & 17.9 & \\
\hline CP.8S_U & 4 May 04 & 24 Apr 06 & 1210 & 380 & 5.80 & 0.0153 & 5.57 & \\
\hline CP.8W_U & 5 May 04 & 24 Apr 06 & 1210 & 380 & 5.70 & 0.0150 & 5.48 & \\
\hline CP.8NW_U & 3 May 05 & 24 Apr 06 & 1210 & 356 & 6.50 & 0.0183 & 6.66 & \\
\hline CP.8N_U & 6 May 04 & 24 Apr 06 & 1210 & 386 & 7.20 & 0.0187 & 6.81 & \\
\hline CS.24E_U & 7 May 04 & 24 Apr 06 & 1210 & 380 & 5.40 & 0.0142 & 5.19 & \\
\hline Mean undisturbed & & & & & & & 10.5 & \\
\hline \multicolumn{8}{|l|}{ Disturbed plots: } & \multirow{12}{*}{$\begin{array}{l}\text { Number } 8 \text { wire out by } 25 \text { October } 2005 \text {. Number } 10 \text { wire disturbed by } 26 \text { April } 2006 \text {. } \\
\text { Established trail, no flags installed for safety issues. } \\
6 \text { August } 2004 \text { installation notes missing, assume } 1,800 \text { mm initial distance. } \\
\text { Number } 10 \text { wire disturbed by footprint before } 9 \text { April } 2005 . \\
\text { Installation measurement error? Reference pin creep? All creep values negative. }\end{array}$} \\
\hline ES.9SE_D & 19 Aug 03 & 26 Apr 06 & 1212 & 364 & 12.7 & 0.0349 & 12.7 & \\
\hline ES.9NW"_D & 22 Aug 03 & 26 Apr 06 & 1212 & 364 & 5.70 & 0.0157 & 5.72 & \\
\hline ES.9SW_D & 13 Aug 04 & 26 Apr 06 & 1212 & 364 & -0.750 & -0.00206 & -0.752 & \\
\hline ES.38NE_D & none & na & na & na & na & na & na & \\
\hline ES.40E_D & 6 Aug 04 & 26 Apr 06 & 1212 & 364 & 3.30 & 0.00907 & 3.31 & \\
\hline ES.40NW_D & 9 Jun 05 & 26 Apr 06 & 1212 & 321 & 13.2 & 0.0411 & 15.0 & \\
\hline CP.8S_D & 10 Aug 04 & 24 Apr 06 & 1210 & 380 & 4.20 & 0.0111 & 4.03 & \\
\hline CP.8W_D & 11 Aug 04 & 24 Apr 06 & 1210 & 380 & 2.70 & 0.0071 & 2.59 & \\
\hline CP.8NW_D & 4 May 05 & 24 Apr 06 & 1210 & na & na & na & na & \\
\hline CP.8N_D & 12 Aug 04 & 24 Apr 06 & 1210 & 386 & -2.30 & -0.00596 & -2.17 & \\
\hline Mean disturbed & & & & & & & 5.06 & \\
\hline
\end{tabular}


Table 6. Hillslope-erosion data from silt-fence traps on undisturbed areas at Gunnison Gorge National Conservation Area, southwestern Colorado.

$\left[\mathrm{m}^{2}\right.$, square meters; $\mathrm{kg}$, kilogram; kg/m², kilograms per square meter; $\mathrm{Mg} / \mathrm{ha}$, megagrams per hectare; $\mathrm{Mg} / \mathrm{ha} / \mathrm{d}$, megagrams per hectare per day; $\mathrm{Mg} / \mathrm{ha} / \mathrm{yr}, \mathrm{megagrams}$ per hectare per year; $\sim$, approximately; na, not applicable or no data]

\begin{tabular}{|c|c|c|c|c|c|c|c|c|c|c|c|c|c|c|}
\hline \multirow[b]{2}{*}{$\begin{array}{c}\text { Hillslope- } \\
\text { erosion plot } \\
\text { identification } \\
\text { code }\end{array}$} & \multirow[b]{2}{*}{$\begin{array}{l}\text { Date silt } \\
\text { fence } \\
\text { installed }\end{array}$} & \multirow[b]{2}{*}{$\begin{array}{l}\text { Julian day } \\
\text { since } \\
\text { January } \\
2004\end{array}$} & \multirow[b]{2}{*}{$\begin{array}{c}\begin{array}{c}\text { Erosion } \\
\text { plot area }\end{array} \\
\left(\mathrm{m}^{2}\right) \\
\end{array}$} & \multirow[b]{2}{*}{$\begin{array}{l}\begin{array}{l}\text { Surveyed } \\
\text { hillslope } \\
\text { gradient }\end{array} \\
\text { (degrees) }\end{array}$} & \multirow[b]{2}{*}{$\begin{array}{c}\begin{array}{c}\text { Erosion } \\
\text { plot } \\
\text { aspect }\end{array} \\
\text { (degrees) } \\
\end{array}$} & \multirow[b]{2}{*}{$\begin{array}{c}\begin{array}{c}\text { Erosion } \\
\text { plot } \\
\text { vegetation } \\
\text { cover }\end{array} \\
\text { (percent) } \\
\end{array}$} & \multicolumn{3}{|c|}{\begin{tabular}{|l} 
Fall and winter 2004 / 2005 \\
(since October 2004, 5 months)
\end{tabular}} & \multirow[b]{2}{*}{$\begin{array}{c}\text { Dry } \\
\text { sediment } \\
\text { weight }^{1}\end{array}$} & \multirow[b]{2}{*}{$\begin{array}{l}\text { Sediment } \\
\text { yield }\end{array}$} & \multirow[b]{2}{*}{$\begin{array}{l}\text { Sediment } \\
\text { yield }\end{array}$} & \multirow[b]{2}{*}{$\begin{array}{c}\text { Daily } \\
\text { erosion rate }\end{array}$} & \multirow[b]{2}{*}{$\begin{array}{c}\text { Seasonal } \\
\text { erosion } \\
\text { rate }\end{array}$} \\
\hline & & & & & & & $\begin{array}{c}\text { Date } \\
\text { observed }\end{array}$ & $\begin{array}{c}\text { Julian } \\
\text { day since } \\
\text { January } \\
2004\end{array}$ & $\begin{array}{l}\text { Elapsed } \\
\text { number } \\
\text { of days }\end{array}$ & & & & & \\
\hline ES.9SE & 20 Oct 04 & 294 & 50 & 36.5 & 163 & 0 & 23 Mar 05 & 448 & 154 & 0.0 & 0.0 & 0.0 & 0.0 & 0.0 \\
\hline ES.9NW & 21 Oct 04 & 295 & 50 & 28.0 & 296 & 15 & 23 Mar 05 & 448 & 153 & 0.0 & 0.0 & 0.0 & 0.0 & 0.0 \\
\hline ES.40E & 7 Jun 05 & 524 & 28 & 33.0 & 103 & 10 & & & & & & & & \\
\hline CP.8S & 6 May 05 & 492 & 28 & 38.0 & 179 & 0 & & & & & & & & \\
\hline CP.8W & 5 May 05 & 491 & 50 & 40.0 & 240 & 0 & & & & & & & & \\
\hline CP.8N & 21 Oct 04 & 295 & 50 & 32.0 & 14 & 10 & 23 Mar 05 & 448 & 153 & 0.0 & 0.0 & 0.0 & 0.0 & 0.0 \\
\hline Mean & & & & & & & & & & & & & & 0.0 \\
\hline
\end{tabular}


Table 6. Hillslope-erosion data from silt-fence traps on undisturbed areas at Gunnison Gorge National Conservation Area, southwestern Colorado.-Continued

$\left[\mathrm{m}^{2}\right.$, square meters; $\mathrm{kg}$, kilogram; $\mathrm{kg} / \mathrm{m}^{2}$, kilograms per square meter; Mg/ha, megagrams per hectare; Mg/ha/d, megagrams per hectare per day; Mg/ha/yr, megagrams per hectare per year; $\sim$, approximately; na, not applicable or no data]

\begin{tabular}{|c|c|c|c|c|c|c|c|c|c|c|c|c|c|c|}
\hline \multirow[b]{2}{*}{$\begin{array}{c}\text { Hillslope- } \\
\text { erosion plot } \\
\text { identification } \\
\text { code }\end{array}$} & \multirow[b]{2}{*}{$\begin{array}{c}\text { Date silt } \\
\text { fence } \\
\text { installed }\end{array}$} & \multirow[b]{2}{*}{$\begin{array}{l}\text { Julian day } \\
\text { since } \\
\text { January } \\
2004\end{array}$} & \multirow[b]{2}{*}{$\begin{array}{c}\begin{array}{c}\text { Erosion } \\
\text { plot area }\end{array} \\
\left(\mathrm{m}^{2}\right) \\
\end{array}$} & \multirow[b]{2}{*}{$\begin{array}{c}\text { Surveyed } \\
\text { hillslope } \\
\text { gradient }\end{array}$} & \multirow[b]{2}{*}{$\begin{array}{c}\begin{array}{c}\text { Erosion } \\
\text { plot } \\
\text { aspect }\end{array} \\
\text { (degrees) }\end{array}$} & \multirow[b]{2}{*}{$\begin{array}{c}\begin{array}{c}\text { Erosion } \\
\text { plot } \\
\text { vegetation } \\
\text { cover }\end{array} \\
\text { (percent) } \\
\end{array}$} & \multicolumn{8}{|c|}{\begin{tabular}{|l|} 
Spring 2005 \\
(since March $2005, \sim 1.5$ to 2.6 months) \\
\end{tabular}} \\
\hline & & & & & & & $\begin{array}{c}\text { Date } \\
\text { observed } \\
\text { or } \\
\text { installed }\end{array}$ & $\begin{array}{c}\text { Julian } \\
\text { day } \\
\text { since } \\
\text { January } \\
2004\end{array}$ & $\begin{array}{l}\text { Elapsed } \\
\text { number } \\
\text { of days }\end{array}$ & $\begin{array}{c}\text { Dry } \\
\text { sediment } \\
\text { weight }^{1,2}\end{array}$ & $\begin{array}{l}\text { Sediment } \\
\text { yield }\end{array}$ & $\begin{array}{l}\text { Sediment } \\
\text { yield }\end{array}$ & $\begin{array}{c}\text { Daily } \\
\text { erosion rate }\end{array}$ & $\begin{array}{l}\text { Seasonal } \\
\text { erosion } \\
\text { rate }\end{array}$ \\
\hline ES.9SE & 20 Oct 04 & 294 & 50 & 36.5 & 163 & 0 & 9 Jun 05 & 526 & 78 & 0.0 & 0.0 & 0.0 & 0.0 & 0.0 \\
\hline ES.9NW & 21 Oct 04 & 295 & 50 & 28.0 & 296 & 15 & 9 Jun 05 & 526 & 78 & 0.464 & 0.00927 & 0.0927 & 0.00119 & 0.434 \\
\hline ES.40E & 7 Jun 05 & 524 & 28 & 33.0 & 103 & 10 & 7 Jun 05 & 524 & 0 & na & na & na & na & na \\
\hline CP.8S & 6 May 05 & 492 & 28 & 38.0 & 179 & 0 & 6 May 05 & 492 & 0 & na & na & na & na & na \\
\hline CP.8W & 5 May 05 & 491 & 50 & 40.0 & 240 & 0 & 5 May 05 & 491 & 0 & na & na & na & na & na \\
\hline CP.8N & 21 Oct 04 & 295 & 50 & 32.0 & 14 & 10 & 6 May 05 & 492 & 44 & 1.10 & 0.0220 & 0.220 & 0.00500 & 1.83 \\
\hline Mean & & & & & & & & & & & & & & 0.753 \\
\hline
\end{tabular}


Table 6. Hillslope-erosion data from silt-fence traps on undisturbed areas at Gunnison Gorge National Conservation Area, southwestern Colorado.—Continued

$\left[\mathrm{m}^{2}\right.$, square meters; $\mathrm{kg}$, kilogram; $\mathrm{kg} / \mathrm{m}^{2}$, kilograms per square meter; $\mathrm{Mg} / \mathrm{ha}$, megagrams per hectare; $\mathrm{Mg} / \mathrm{ha} / \mathrm{d}$, megagrams per hectare per day; $\mathrm{Mg} / \mathrm{ha} / \mathrm{yr}$, megagrams per hectare per year; $\sim$, approximately; na, not applicable or no data]

\begin{tabular}{|c|c|c|c|c|c|c|c|c|c|c|c|c|c|c|}
\hline \multirow[b]{2}{*}{$\begin{array}{c}\text { Hillslope- } \\
\text { erosion plot } \\
\text { identification } \\
\text { code }\end{array}$} & \multirow[b]{2}{*}{$\begin{array}{l}\text { Date silt } \\
\text { fence } \\
\text { installed }\end{array}$} & \multirow[b]{2}{*}{$\begin{array}{l}\text { Julian day } \\
\text { since } \\
\text { January } \\
2004\end{array}$} & \multirow[b]{2}{*}{$\begin{array}{c}\begin{array}{c}\text { Erosion } \\
\text { plot area }\end{array} \\
\left(\mathrm{m}^{2}\right)\end{array}$} & \multirow[b]{2}{*}{$\begin{array}{l}\begin{array}{l}\text { Surveyed } \\
\text { hillslope } \\
\text { gradient }\end{array} \\
\text { (degrees) }\end{array}$} & \multirow[b]{2}{*}{$\begin{array}{c}\begin{array}{c}\text { Erosion } \\
\text { plot } \\
\text { aspect }\end{array} \\
\text { (degrees) }\end{array}$} & \multirow[b]{2}{*}{$\begin{array}{c}\begin{array}{c}\text { Erosion } \\
\text { plot } \\
\text { vegetation } \\
\text { cover }\end{array} \\
\text { (percent) } \\
\end{array}$} & \multicolumn{8}{|c|}{$\begin{array}{l}\text { Summer } 2005 \\
\text { (since May } 2005 \sim 5.8 \text { months; since June } 2005 \sim 4.6 \text { months) }\end{array}$} \\
\hline & & & & & & & $\begin{array}{c}\text { Date } \\
\text { observed }\end{array}$ & $\begin{array}{c}\text { Julian } \\
\text { day } \\
\text { since } \\
\text { January } \\
2004\end{array}$ & $\begin{array}{l}\text { Elapsed } \\
\text { number } \\
\text { of days }\end{array}$ & $\begin{array}{c}\text { Dry } \\
\text { sediment } \\
\text { weight }^{1,2,3}\end{array}$ & $\begin{array}{l}\text { Sediment } \\
\text { yield }\end{array}$ & $\begin{array}{l}\text { Sediment } \\
\text { yield }\end{array}$ & $\begin{array}{l}\text { Daily } \\
\text { erosion } \\
\text { rate }\end{array}$ & $\begin{array}{l}\text { Seasonal } \\
\text { erosion rate }\end{array}$ \\
\hline ES.9SE & 20 Oct 04 & 294 & 50 & 36.5 & 163 & 0 & 25 Oct 05 & 664 & 138 & 3.24 & 0.0648 & 0.648 & 0.00470 & 1.72 \\
\hline ES.9NW & 21 Oct 04 & 295 & 50 & 28.0 & 296 & 15 & 25 Oct 05 & 664 & 138 & 60.9 & 1.22 & 12.2 & 0.0882 & 32.2 \\
\hline ES.40E & 7 Jun 05 & 524 & 28 & 33.0 & 103 & 10 & 25 Oct 05 & 664 & 140 & 2.92 & 0.104 & 1.04 & 0.00744 & 2.72 \\
\hline CP.8S & 6 May 05 & 492 & 28 & 38.0 & 179 & 0 & 26 Oct 05 & 665 & 173 & 8.19 & 0.293 & 2.93 & 0.0169 & 6.17 \\
\hline CP.8W & 5 May 05 & 491 & 50 & 40.0 & 240 & 0 & 26 Oct 05 & 665 & 174 & 34.0 & 0.680 & 6.80 & 0.0391 & 14.3 \\
\hline CP.8N & 21 Oct 04 & 295 & 50 & 32.0 & 14 & 10 & 26 Oct 05 & 665 & 173 & 0.875 & 0.0175 & 0.175 & 0.00101 & 0.369 \\
\hline Mean & & & & & & & & & & & & & & 9.57 \\
\hline
\end{tabular}


Table 6. Hillslope-erosion data from silt-fence traps on undisturbed areas at Gunnison Gorge National Conservation Area, southwestern Colorado.-Continued $\left[\mathrm{m}^{2}\right.$, square meters; $\mathrm{kg}$, kilogram; kg/m², kilograms per square meter; Mg/ha, megagrams per hectare; Mg/ha/d, megagrams per hectare per day; Mg/ha/yr, megagrams per hectare per year; , approximately; na, not applicable or no data]

\begin{tabular}{|c|c|c|c|c|c|c|c|c|c|c|c|c|c|c|}
\hline \multirow[b]{2}{*}{$\begin{array}{c}\text { Hillslope- } \\
\text { erosion plot } \\
\text { identification } \\
\text { code }\end{array}$} & \multirow[b]{2}{*}{$\begin{array}{c}\text { Date silt } \\
\text { fence } \\
\text { installed }\end{array}$} & \multirow[b]{2}{*}{$\begin{array}{l}\text { Julian day } \\
\text { since } \\
\text { January } \\
2004\end{array}$} & \multirow[b]{2}{*}{$\begin{array}{c}\begin{array}{c}\text { Erosion } \\
\text { plot area }\end{array} \\
\left(\mathrm{m}^{2}\right)\end{array}$} & \multirow[b]{2}{*}{$\begin{array}{l}\begin{array}{c}\text { Surveyed } \\
\text { hillslope } \\
\text { gradient }\end{array} \\
\text { (degrees) }\end{array}$} & \multirow[b]{2}{*}{$\begin{array}{c}\begin{array}{c}\text { Erosion } \\
\text { plot } \\
\text { aspect }\end{array} \\
\text { (degrees) }\end{array}$} & \multirow[b]{2}{*}{$\begin{array}{c}\begin{array}{c}\text { Erosion } \\
\text { plot } \\
\text { vegetation } \\
\text { cover }\end{array} \\
\text { (percent) }\end{array}$} & \multicolumn{8}{|c|}{$\begin{array}{l}\text { Fall and winter } 2005 \text { / } 2006 \\
\text { (since October } 20056 \text { mos) }\end{array}$} \\
\hline & & & & & & & $\begin{array}{c}\text { Date } \\
\text { observed }\end{array}$ & $\begin{array}{c}\text { Julian } \\
\text { day } \\
\text { since } \\
\text { January } \\
2004\end{array}$ & $\begin{array}{c}\text { Elapsed } \\
\text { number } \\
\text { of days }\end{array}$ & $\begin{array}{c}\text { Dry } \\
\text { sediment } \\
\text { weight }^{1,2,3}\end{array}$ & $\begin{array}{c}\text { Sediment } \\
\text { yield }\end{array}$ & $\begin{array}{l}\text { Sediment } \\
\text { yield }\end{array}$ & $\begin{array}{c}\text { Daily } \\
\text { erosion rate }\end{array}$ & $\begin{array}{c}\begin{array}{c}\text { Seasonal } \\
\text { erosion } \\
\text { rate }\end{array} \\
\text { (Mg/ha/yr) }\end{array}$ \\
\hline ES.9SE & 20 Oct 04 & 294 & 50 & 36.5 & 163 & 0 & 26 Apr 06 & 847 & 183 & 7.50 & 0.150 & 1.50 & 0.00820 & 2.99 \\
\hline ES.9NW & 21 Oct 04 & 295 & 50 & 28.0 & 296 & 15 & 26 Apr 06 & 847 & 183 & 2.01 & 0.0403 & 0.403 & 0.00220 & 0.803 \\
\hline ES.40E & 7 Jun 05 & 524 & 28 & 33.0 & 103 & 10 & 26 Apr 06 & 847 & 183 & 0.568 & 0.0203 & 0.203 & 0.00111 & 0.405 \\
\hline CP.8S & 6 May 05 & 492 & 28 & 38.0 & 179 & 0 & 25 Apr 06 & 846 & 181 & 11.6 & 0.414 & 4.14 & 0.0229 & 8.35 \\
\hline CP.8W & 5 May 05 & 491 & 50 & 40.0 & 240 & 0 & 25 Apr 06 & 846 & 181 & 26.0 & 0.520 & 5.20 & 0.0287 & 10.5 \\
\hline CP.8N & 21 Oct 04 & 295 & 50 & 32.0 & 14 & 10 & 25 Apr 06 & 846 & 181 & na & na & na & na & na \\
\hline Mean & & & & & & & & & & & & & & 4.61 \\
\hline
\end{tabular}

${ }^{1}$ Dry sediment weights—-Samples were collected (1) dry onsite, (2) air dried for several weeks in the laboratory, or (3) weight adjusted by using a moisture correction factor determined from an air-dried subsample.

${ }^{2}$ Yellow highlighted weights-All captured sediment was collected onsite, dried, and weighted in the laboratory to the nearest $0.001 \mathrm{~kg}$.

${ }^{3}$ Pink highlighted weights-All captured sediment was collected and weighted onsite to the nearest $0.0 \mathrm{~kg}$. If the field sample was large and moist, a subsample of captured sediment was dried and weighted in laboratory to the nearest $0.001 \mathrm{~kg}$. A moisture correction factor from the dried subsample was applied to the field sample to determine a dry sediment weight to the nearest $0.1 \mathrm{~kg}$. 
Table 7. Daily and cumulative precipitation at the Montrose \#2 climate station, upper Elephant Skin Wash, and Candy Lane rain gages, southwestern Colorado.

[in., inch; mm, millimeter; md, missing data due to equipment malfunction or vandalism; MA, Montrose \#2 climate station; ES, upper Elephant Skin Wash; CL, Candy Lane]

\begin{tabular}{|c|c|c|c|c|c|c|c|c|c|c|c|c|c|}
\hline \multirow[b]{2}{*}{$\begin{array}{c}\text { Julian day } \\
\text { since } 1 \\
\text { January } \\
2003\end{array}$} & \multicolumn{4}{|c|}{ Montrose \#2 climate station rain gage } & \multicolumn{4}{|c|}{ Upper Elephant Skin Wash rain gage } & \multicolumn{4}{|c|}{ Candy Lane rain gage } & \multirow[b]{2}{*}{ Remark } \\
\hline & $\begin{array}{l}\text { Precip- } \\
\text { itation } \\
\text { (in.) } \\
\end{array}$ & $\begin{array}{c}\text { Cumu- } \\
\text { lative } \\
\text { precip- } \\
\text { itation } \\
\text { (in.) }\end{array}$ & $\begin{array}{l}\text { Precip- } \\
\text { itation } \\
(\mathrm{mm})\end{array}$ & $\begin{array}{c}\text { Cumu- } \\
\text { lative } \\
\text { precip- } \\
\text { itation } \\
(\mathrm{mm})\end{array}$ & $\begin{array}{c}\text { Precip- } \\
\text { itation } \\
\text { (in.) } \\
\end{array}$ & $\begin{array}{c}\text { Cumu- } \\
\text { lative } \\
\text { precip- } \\
\text { itation } \\
\text { (in.) }\end{array}$ & $\begin{array}{l}\text { Precip- } \\
\text { itation } \\
(\mathrm{mm})\end{array}$ & $\begin{array}{c}\text { Cumu- } \\
\text { lative } \\
\text { precip- } \\
\text { itation } \\
(\mathrm{mm})\end{array}$ & $\begin{array}{c}\text { Precip- } \\
\text { itation } \\
\text { (in.) } \\
\end{array}$ & $\begin{array}{c}\text { Cumu- } \\
\text { lative } \\
\text { precip- } \\
\text { itation } \\
\text { (in.) }\end{array}$ & $\begin{array}{l}\text { Precip- } \\
\text { itation } \\
(\mathrm{mm}) \\
\end{array}$ & $\begin{array}{c}\text { Cumu- } \\
\text { lative } \\
\text { precip- } \\
\text { itation } \\
(\mathrm{mm})\end{array}$ & \\
\hline 213 & 0.00 & 0.00 & 0.00 & 0 & & & & & & & & & 1 August 2003 \\
\hline 214 & 0.00 & 0.00 & 0.00 & 0 & & & & & & & & & \\
\hline 215 & 0.00 & 0.00 & 0.00 & 0 & & & & & & & & & \\
\hline 216 & 0.10 & 0.10 & 2.54 & 3 & & & & & & & & & \\
\hline 217 & 0.00 & 0.10 & 0.00 & 3 & & & & & & & & & \\
\hline 218 & 0.00 & 0.10 & 0.00 & 3 & & & & & & & & & \\
\hline 219 & 0.02 & 0.12 & 0.51 & 3 & & & & & & & & & \\
\hline 220 & 0.03 & 0.15 & 0.76 & 4 & & & & & & & & & \\
\hline 221 & 0.00 & 0.15 & 0.00 & 4 & & & & & & & & & \\
\hline 222 & 0.00 & 0.15 & 0.00 & 4 & & & & & & & & & \\
\hline 223 & 0.00 & 0.15 & 0.00 & 4 & & & & & & & & & \\
\hline 224 & 0.00 & 0.15 & 0.00 & 4 & & & & & & & & & \\
\hline 225 & 0.00 & 0.15 & 0.00 & 4 & & & & & & & & & \\
\hline 226 & 0.00 & 0.15 & 0.00 & 4 & & & & & & & & & \\
\hline 227 & 0.26 & 0.41 & 6.60 & 10 & & & & & & & & & \\
\hline 228 & 0.00 & 0.41 & 0.00 & 10 & & & & & & & & & \\
\hline 229 & 0.00 & 0.41 & 0.00 & 10 & & & & & & & & & \\
\hline 230 & 0.05 & 0.46 & 1.27 & 12 & & & & & & & & & \\
\hline 231 & 0.00 & 0.46 & 0.00 & 12 & & & & & & & & & \\
\hline 232 & 0.00 & 0.46 & 0.00 & 12 & & & & & & & & & \\
\hline 233 & 0.00 & 0.46 & 0.00 & 12 & & & & & & & & & \\
\hline 234 & Trace & 0.46 & Trace & 12 & & & & & & & & & \\
\hline 235 & 0.13 & 0.59 & 3.30 & 15 & & & & & & & & & \\
\hline 236 & 0.00 & 0.59 & 0.00 & 15 & & & & & & & & & \\
\hline 237 & 0.00 & 0.59 & 0.00 & 15 & & & & & & & & & \\
\hline 238 & 0.06 & 0.65 & 1.52 & 17 & & & & & & & & & \\
\hline 239 & 0.00 & 0.65 & 0.00 & 17 & & & & & & & & & \\
\hline 240 & 0.19 & 0.84 & 4.83 & 21 & & & & & & & & & \\
\hline 241 & 0.00 & 0.84 & 0.00 & 21 & & & & & & & & & \\
\hline 242 & 0.00 & 0.84 & 0.00 & 21 & & & & & & & & & \\
\hline 243 & 0.05 & 0.89 & 1.27 & 23 & & & & & & & & & \\
\hline 244 & 0.00 & 0.89 & 0.00 & 23 & & & & & & & & & 1 September 2003 \\
\hline 245 & 0.00 & 0.89 & 0.00 & 23 & & & & & & & & & \\
\hline 246 & Trace & 0.90 & Trace & 23 & & & & & & & & & \\
\hline 247 & 0.00 & 0.90 & 0.00 & 23 & & & & & & & & & \\
\hline 248 & 0.00 & 0.90 & 0.00 & 23 & & & & & & & & & \\
\hline 249 & 0.33 & 1.23 & 8.38 & 31 & & & & & & & & & \\
\hline 250 & 0.20 & 1.43 & 5.08 & 36 & & & & & & & & & \\
\hline 251 & Trace & 1.43 & Trace & 36 & & & & & & & & & \\
\hline 252 & 0.00 & 1.43 & 0.00 & 36 & & & & & & & & & \\
\hline 253 & 0.85 & 2.28 & 21.59 & 58 & & & & & & & & & \\
\hline 254 & 0.39 & 2.67 & 9.91 & 68 & & & & & & & & & \\
\hline 255 & 0.00 & 2.67 & 0.00 & 68 & & & & & & & & & \\
\hline 256 & 0.00 & 2.67 & 0.00 & 68 & & & & & & & & & \\
\hline 257 & 0.00 & 2.67 & 0.00 & 68 & & & & & & & & & \\
\hline
\end{tabular}


Table 7. Daily and cumulative precipitation at the Montrose \#2 climate station, upper Elephant Skin Wash, and Candy Lane rain gages, southwestern

Colorado.-Continued

\begin{tabular}{|c|c|c|c|c|c|c|c|c|c|c|c|c|c|}
\hline \multirow[b]{2}{*}{$\begin{array}{c}\text { Julian day } \\
\text { since } 1 \\
\text { January } \\
2003\end{array}$} & \multicolumn{4}{|c|}{ Montrose \#2 climate station rain gage } & \multicolumn{4}{|c|}{ Upper Elephant Skin Wash rain gage } & \multicolumn{4}{|c|}{ Candy Lane rain gage } & \multirow[b]{2}{*}{ Remark } \\
\hline & $\begin{array}{l}\text { Precip- } \\
\text { itation } \\
\text { (in.) } \\
\end{array}$ & $\begin{array}{l}\text { Cumu- } \\
\text { lative } \\
\text { precip- } \\
\text { itation } \\
\text { (in.) }\end{array}$ & $\begin{array}{l}\text { Precip- } \\
\text { itation } \\
(\mathrm{mm})\end{array}$ & $\begin{array}{l}\text { Cumu- } \\
\text { lative } \\
\text { precip- } \\
\text { itation } \\
(\mathrm{mm})\end{array}$ & $\begin{array}{l}\begin{array}{l}\text { Precip- } \\
\text { itation }\end{array} \\
\text { (in.) } \\
\end{array}$ & $\begin{array}{l}\text { Cumu- } \\
\text { lative } \\
\text { precip- } \\
\text { itation } \\
\text { (in.) }\end{array}$ & $\begin{array}{l}\text { Precip- } \\
\text { itation } \\
(\mathrm{mm})\end{array}$ & $\begin{array}{l}\text { Cumu- } \\
\text { lative } \\
\text { precip- } \\
\text { itation } \\
(\mathrm{mm})\end{array}$ & $\begin{array}{l}\text { Precip- } \\
\text { itation } \\
\text { (in.) } \\
\end{array}$ & $\begin{array}{l}\text { Cumu- } \\
\text { lative } \\
\text { precip- } \\
\text { itation } \\
\text { (in.) }\end{array}$ & $\begin{array}{l}\text { Precip- } \\
\text { itation } \\
(\mathrm{mm})\end{array}$ & $\begin{array}{l}\text { Cumu- } \\
\text { lative } \\
\text { precip- } \\
\text { itation } \\
(\mathrm{mm})\end{array}$ & \\
\hline 258 & 0.00 & 2.67 & 0.00 & 68 & & & & & & & & & \\
\hline 259 & 0.00 & 2.67 & 0.00 & 68 & & & & & & & & & \\
\hline 261 & 0.00 & 2.67 & 0.00 & 68 & & & & & & & & & \\
\hline 262 & 0.00 & 2.67 & 0.00 & 68 & & & & & & & & & \\
\hline 263 & 0.00 & 2.67 & 0.00 & 68 & & & & & & & & & \\
\hline 264 & 0.00 & 2.67 & 0.00 & 68 & & & & & & & & & \\
\hline 265 & 0.00 & 2.67 & 0.00 & 68 & & & & & & & & & \\
\hline 266 & 0.00 & 2.67 & 0.00 & 68 & & & & & & & & & \\
\hline 267 & 0.00 & 2.67 & 0.00 & 68 & & & & & & & & & \\
\hline 268 & 0.00 & 2.67 & 0.00 & 68 & & & & & & & & & \\
\hline 269 & 0.00 & 2.67 & 0.00 & 68 & & & & & & & & & \\
\hline 270 & 0.00 & 2.67 & 0.00 & 68 & & & & & & & & & \\
\hline 273 & 0.00 & 2.67 & 0.00 & 68 & & & & & & & & & \\
\hline 274 & 0.00 & 2.67 & 0.00 & 68 & & & & & & & & & 1 October 2003 \\
\hline 275 & 0.03 & 2.70 & 0.76 & 69 & & & & & & & & & \\
\hline 276 & 0.28 & 2.98 & 7.11 & 76 & & & & & & & & & \\
\hline 277 & 0.20 & 3.18 & 5.08 & 81 & & & & & & & & & \\
\hline 278 & 0.00 & 3.18 & 0.00 & 81 & & & & & & & & & \\
\hline 279 & 0.00 & 3.18 & 0.00 & 81 & & & & & & & & & \\
\hline 280 & 0.00 & 3.18 & 0.00 & 81 & & & & & & & & & \\
\hline 281 & 0.00 & 3.18 & 0.00 & 81 & & & & & & & & & \\
\hline 282 & 0.00 & 3.18 & 0.00 & 81 & & & & & & & & & \\
\hline 283 & 0.00 & 3.18 & 0.00 & 81 & & & & & & & & & \\
\hline 284 & 0.15 & 3.33 & 3.81 & 85 & & & & & & & & & \\
\hline 285 & 0.00 & 3.33 & 0.00 & 85 & & & & & & & & & \\
\hline 286 & 0.00 & 3.33 & 0.00 & 85 & & & & & & & & & \\
\hline 287 & 0.00 & 3.33 & 0.00 & 85 & & & & & & & & & \\
\hline 288 & 0.00 & 3.33 & 0.00 & 85 & & & & & & & & & \\
\hline 289 & 0.00 & 3.33 & 0.00 & 85 & & & & & & & & & \\
\hline 290 & 0.00 & 3.33 & 0.00 & 85 & & & & & & & & & \\
\hline 291 & 0.00 & 3.33 & 0.00 & 85 & & & & & & & & & \\
\hline 292 & 0.00 & 3.33 & 0.00 & 85 & & & & & & & & & \\
\hline 296 & 0.00 & 3.33 & 0.00 & 85 & & & & & & & & & \\
\hline 297 & 0.00 & 3.33 & 0.00 & 85 & & & & & & & & & \\
\hline 298 & 0.00 & 3.33 & 0.00 & 85 & & & & & & & & & \\
\hline 299 & 0.00 & 3.33 & 0.00 & 85 & & & & & & & & & \\
\hline 300 & 0.00 & 3.33 & 0.00 & 85 & & & & & & & & & \\
\hline 301 & 0.00 & 3.33 & 0.00 & 85 & & & & & & & & & \\
\hline 302 & 0.00 & 3.33 & 0.00 & 85 & & & & & & & & & \\
\hline
\end{tabular}


Table 7. Daily and cumulative precipitation at the Montrose \#2 climate station, upper Elephant Skin Wash, and Candy Lane rain gages, southwestern Colorado.-Continued

[in., inch; mm, millimeter; md, missing data due to equipment malfunction or vandalism; MA, Montrose \#2 climate station; ES, upper Elephant Skin Wash; CL, Candy Lane]

\begin{tabular}{|c|c|c|c|c|c|c|c|c|c|c|c|c|c|c|}
\hline \multirow[b]{2}{*}{$\begin{array}{c}\text { Julian day } \\
\text { since } 1 \\
\text { January } \\
2003\end{array}$} & \multicolumn{4}{|c|}{ Montrose \#2 climate station rain gage } & \multicolumn{4}{|c|}{ Upper Elephant Skin Wash rain gage } & \multicolumn{4}{|c|}{ Candy Lane rain gage } & & \multirow[b]{2}{*}{ Remark } \\
\hline & $\begin{array}{l}\text { Precip- } \\
\text { itation } \\
\text { (in.) } \\
\end{array}$ & $\begin{array}{l}\text { Cumu- } \\
\text { lative } \\
\text { precip- } \\
\text { itation } \\
\text { (in.) }\end{array}$ & $\begin{array}{l}\text { Precip- } \\
\text { itation } \\
(\mathrm{mm}) \\
\end{array}$ & $\begin{array}{l}\text { Cumu- } \\
\text { lative } \\
\text { precip- } \\
\text { itation } \\
(\mathrm{mm})\end{array}$ & $\begin{array}{l}\text { Precip- } \\
\text { itation } \\
\text { (in.) }\end{array}$ & $\begin{array}{l}\text { Cumu- } \\
\text { lative } \\
\text { precip- } \\
\text { itation } \\
\text { (in.) }\end{array}$ & $\begin{array}{l}\text { Precip- } \\
\text { itation } \\
(\mathrm{mm})\end{array}$ & $\begin{array}{l}\text { Cumu- } \\
\text { lative } \\
\text { precip- } \\
\text { itation } \\
(\mathrm{mm})\end{array}$ & $\begin{array}{l}\text { Precip- } \\
\text { itation } \\
\text { (in.) } \\
\end{array}$ & $\begin{array}{l}\text { Cumu- } \\
\text { lative } \\
\text { precip- } \\
\text { itation } \\
\text { (in.) }\end{array}$ & $\begin{array}{l}\text { Precip- } \\
\text { itation } \\
(\mathrm{mm})\end{array}$ & $\begin{array}{c}\text { Cumu- } \\
\text { lative } \\
\text { precip- } \\
\text { itation } \\
(\mathrm{mm})\end{array}$ & & \\
\hline 303 & 0.00 & 3.33 & 0.00 & 85 & & & & & & & & & & \\
\hline 304 & 0.00 & 3.33 & 0.00 & 85 & & & & & & & & & & \\
\hline 305 & 0.00 & 3.33 & 0.00 & 85 & & & & & & & & & 1 November 2003 & \\
\hline 306 & Trace & 3.34 & Trace & 85 & & & & & & & & & & \\
\hline 307 & 0.10 & 3.44 & 2.54 & 87 & & & & & & & & & & \\
\hline 308 & 0.00 & 3.44 & 0.00 & 87 & & & & & & & & & & \\
\hline 309 & 0.00 & 3.44 & 0.00 & 87 & & & & & & & & & & \\
\hline 310 & 0.00 & 3.44 & 0.00 & 87 & & & & & & & & & & \\
\hline 311 & 0.00 & 3.44 & 0.00 & 87 & & & & & & & & & & \\
\hline 312 & 0.00 & 3.44 & 0.00 & 87 & & & & & & & & & & \\
\hline 313 & 0.00 & 3.44 & 0.00 & 87 & & & & & & & & & & \\
\hline 314 & 0.10 & 3.54 & 2.54 & 90 & & & & & & & & & & \\
\hline 315 & 0.03 & 3.57 & 0.76 & 91 & & & & & & & & & & \\
\hline 316 & 0.00 & 3.57 & 0.00 & 91 & & & & & & & & & & \\
\hline 317 & 0.05 & 3.62 & 1.27 & 92 & & & & & & & & & & \\
\hline 318 & 0.15 & 3.77 & 3.81 & 96 & & & & & & & & & & \\
\hline 319 & 0.00 & 3.77 & 0.00 & 96 & & & & & & & & & & \\
\hline 320 & 0.00 & 3.77 & 0.00 & 96 & & & & & & & & & & \\
\hline 321 & 0.00 & 3.77 & 0.00 & 96 & & & & & & & & & & \\
\hline 322 & 0.06 & 3.83 & 1.52 & 97 & & & & & & & & & & \\
\hline 323 & 0.00 & 3.83 & 0.00 & 97 & & & & & & & & & & \\
\hline 324 & 0.00 & 3.83 & 0.00 & 97 & & & & & & & & & & \\
\hline 325 & 0.00 & 3.83 & 0.00 & 97 & & & & & & & & & & \\
\hline 326 & 0.00 & 3.83 & 0.00 & 97 & & & & & & & & & & \\
\hline 327 & 0.11 & 3.94 & 2.79 & 100 & & & & & & & & & & \\
\hline 328 & 0.00 & 3.94 & 0.00 & 100 & & & & & & & & & & \\
\hline 329 & 0.00 & 3.94 & 0.00 & 100 & & & & & & & & & & \\
\hline 330 & Trace & 3.94 & Trace & 100 & & & & & & & & & & \\
\hline 331 & 0.00 & 3.94 & 0.00 & 100 & & & & & & & & & & \\
\hline 332 & 0.00 & 3.94 & 0.00 & 100 & & & & & & & & & & \\
\hline 333 & 0.00 & 3.94 & 0.00 & 100 & & & & & & & & & & \\
\hline 334 & 0.00 & 3.94 & 0.00 & 100 & & & & & & & & & & \\
\hline 335 & 0.00 & 3.94 & 0.00 & 100 & & & & & & & & & 1 December 2003 & \\
\hline 336 & 0.00 & 3.94 & 0.00 & 100 & & & & & & & & & & \\
\hline 337 & 0.00 & 3.94 & 0.00 & 100 & & & & & & & & & & \\
\hline 338 & 0.00 & 3.94 & 0.00 & 100 & & & & & & & & & & \\
\hline 339 & 0.00 & 3.94 & 0.00 & 100 & & & & & & & & & & \\
\hline 340 & 0.00 & 3.94 & 0.00 & 100 & & & & & & & & & & \\
\hline 341 & 0.00 & 3.94 & 0.00 & 100 & & & & & & & & & & \\
\hline 342 & 0.21 & 4.15 & 5.33 & 105 & & & & & & & & & & \\
\hline 343 & 0.16 & 4.31 & 4.06 & 109 & & & & & & & & & & \\
\hline 344 & 0.00 & 4.31 & 0.00 & 109 & & & & & & & & & & \\
\hline 345 & 0.00 & 4.31 & 0.00 & 109 & & & & & & & & & & \\
\hline 346 & 0.00 & 4.31 & 0.00 & 109 & & & & & & & & & & \\
\hline 347 & 0.00 & 4.31 & 0.00 & 109 & & & & & & & & & & \\
\hline
\end{tabular}


Table 7. Daily and cumulative precipitation at the Montrose \#2 climate station, upper Elephant Skin Wash, and Candy Lane rain gages, southwestern Colorado.-Continued

[in., inch; mm, millimeter; md, missing data due to equipment malfunction or vandalism; MA, Montrose \#2 climate station; ES, upper Elephant Skin Wash; CL, Candy Lane]

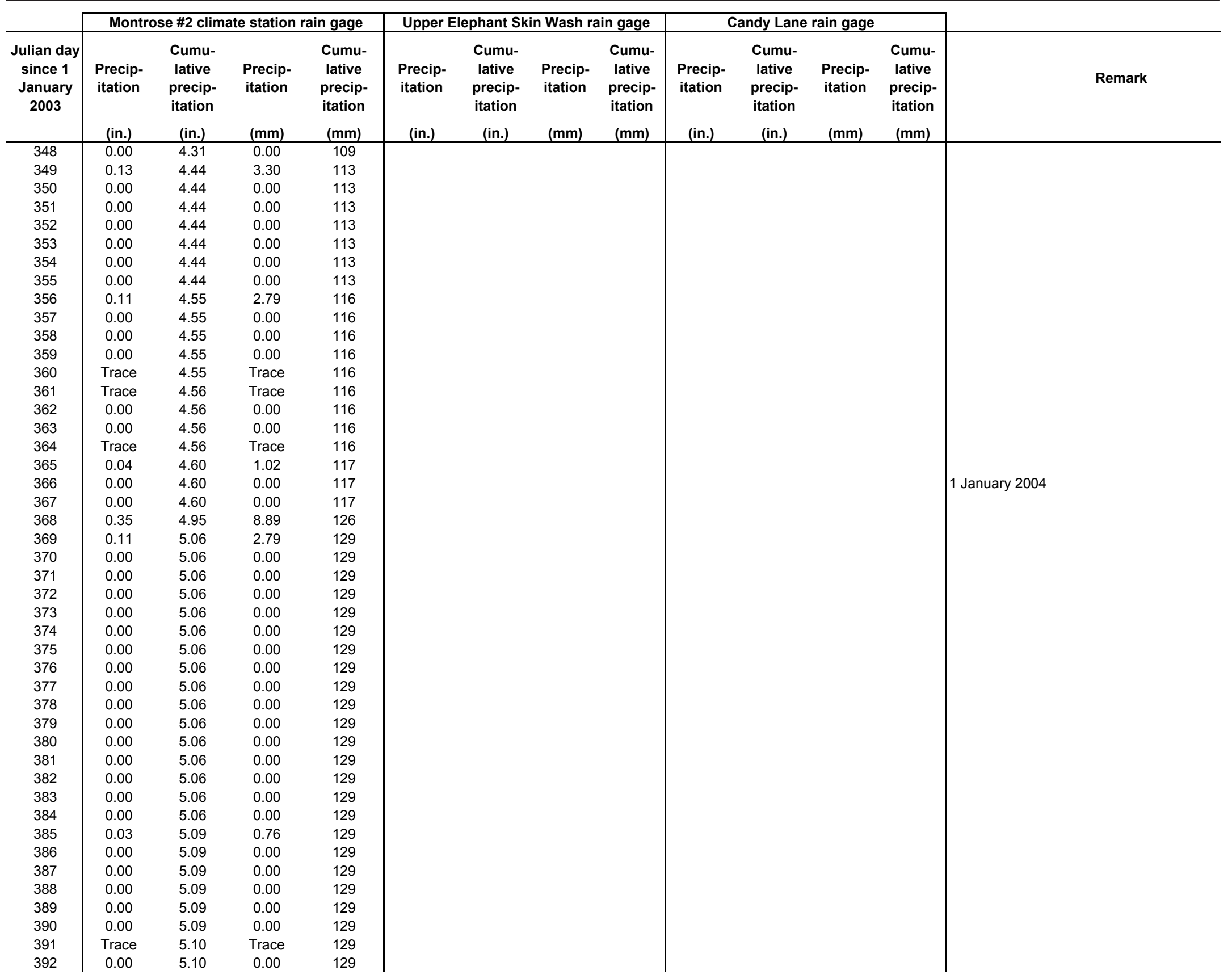


Table 7. Daily and cumulative precipitation at the Montrose \#2 climate station, upper Elephant Skin Wash, and Candy Lane rain gages, southwestern Colorado.-Continued

[in., inch; mm, millimeter; md, missing data due to equipment malfunction or vandalism; MA, Montrose \#2 climate station; ES, upper Elephant Skin Wash; CL, Candy Lane]

\begin{tabular}{|c|c|c|c|c|c|c|c|c|c|c|c|c|c|}
\hline \multirow[b]{2}{*}{$\begin{array}{c}\text { Julian day } \\
\text { since } 1 \\
\text { January } \\
2003\end{array}$} & \multicolumn{4}{|c|}{ Montrose \#2 climate station rain gage } & \multicolumn{4}{|c|}{ Upper Elephant Skin Wash rain gage } & \multicolumn{4}{|c|}{ Candy Lane rain gage } & \multirow[b]{2}{*}{ Remark } \\
\hline & $\begin{array}{l}\text { Precip- } \\
\text { itation } \\
\text { (in.) } \\
\end{array}$ & $\begin{array}{l}\text { Cumu- } \\
\text { lative } \\
\text { precip- } \\
\text { itation } \\
\text { (in.) }\end{array}$ & $\begin{array}{l}\text { Precip- } \\
\text { itation } \\
(\mathrm{mm}) \\
\end{array}$ & $\begin{array}{l}\text { Cumu- } \\
\text { lative } \\
\text { precip- } \\
\text { itation } \\
(\mathrm{mm})\end{array}$ & $\begin{array}{l}\text { Precip- } \\
\text { itation } \\
\text { (in.) } \\
\end{array}$ & $\begin{array}{l}\text { Cumu- } \\
\text { lative } \\
\text { precip- } \\
\text { itation } \\
\text { (in.) }\end{array}$ & $\begin{array}{l}\text { Precip- } \\
\text { itation } \\
(\mathrm{mm}) \\
\end{array}$ & $\begin{array}{l}\text { Cumu- } \\
\text { lative } \\
\text { precip- } \\
\text { itation } \\
(\mathrm{mm})\end{array}$ & $\begin{array}{l}\text { Precip- } \\
\text { itation } \\
\text { (in.) } \\
\end{array}$ & $\begin{array}{l}\text { Cumu- } \\
\text { lative } \\
\text { precip- } \\
\text { itation } \\
\text { (in.) }\end{array}$ & $\begin{array}{l}\text { Precip- } \\
\text { itation } \\
(\mathrm{mm}) \\
\end{array}$ & $\begin{array}{c}\text { Cumu- } \\
\text { lative } \\
\text { precip- } \\
\text { itation } \\
(\mathrm{mm}) \\
\end{array}$ & \\
\hline 393 & 0.00 & 5.10 & 0.00 & 129 & & & & & & & & & \\
\hline 394 & 0.00 & 5.10 & 0.00 & 129 & & & & & & & & & \\
\hline 395 & 0.00 & 5.10 & 0.00 & 129 & & & & & & & & & \\
\hline 397 & 0.20 & 5.30 & 5.08 & 135 & & & & & & & & & 1 February 2004 \\
\hline 398 & 0.00 & 5.30 & 0.00 & 135 & & & & & & & & & \\
\hline 399 & 0.00 & 5.30 & 0.00 & 135 & & & & & & & & & \\
\hline 400 & 0.13 & 5.43 & 3.30 & 138 & & & & & & & & & \\
\hline 401 & 0.13 & 5.56 & 3.30 & 141 & & & & & & & & & \\
\hline 402 & 0.00 & 5.56 & 0.00 & 141 & & & & & & & & & \\
\hline 403 & 0.00 & 5.56 & 0.00 & 141 & & & & & & & & & \\
\hline 404 & 0.00 & 5.56 & 0.00 & 141 & & & & & & & & & \\
\hline 405 & 0.00 & 5.56 & 0.00 & 141 & & & & & & & & & \\
\hline 406 & 0.00 & 5.56 & 0.00 & 141 & & & & & & & & & \\
\hline 409 & 0.00 & 5.59 & 0.00 & 142 & & & & & & & & & \\
\hline 410 & 0.00 & 5.59 & 0.00 & 142 & & & & & & & & & \\
\hline 411 & 0.00 & 5.59 & 0.00 & 142 & & & & & & & & & \\
\hline 412 & 0.00 & 5.59 & 0.00 & 142 & & & & & & & & & \\
\hline 413 & 0.00 & 5.59 & 0.00 & 142 & & & & & & & & & \\
\hline 414 & 0.00 & 5.59 & 0.00 & 142 & & & & & & & & & \\
\hline 415 & 0.00 & 5.59 & 0.00 & 142 & & & & & & & & & \\
\hline 416 & 0.22 & 5.81 & 5.59 & 147 & & & & & & & & & \\
\hline 417 & 0.00 & 5.81 & 0.00 & 147 & & & & & & & & & \\
\hline 418 & 0.05 & 5.86 & 1.27 & 149 & & & & & & & & & \\
\hline 419 & 0.00 & 5.86 & 0.00 & 149 & & & & & & & & & \\
\hline 420 & 0.00 & 5.86 & 0.00 & 149 & & & & & & & & & \\
\hline 421 & 0.00 & 5.86 & 0.00 & 149 & & & & & & & & & \\
\hline 422 & 0.00 & 5.86 & 0.00 & 149 & & & & & & & & & \\
\hline 423 & 0.00 & 5.86 & 0.00 & 149 & & & & & & & & & \\
\hline 424 & 0.20 & 6.06 & 5.08 & 154 & & & & & & & & & \\
\hline 425 & Trace & 6.06 & Trace & 154 & & & & & & & & & \\
\hline 426 & 0.00 & 6.06 & 0.00 & 154 & & & & & & & & & 1 March 2004 \\
\hline 427 & 0.00 & 6.06 & 0.00 & 154 & & & & & & & & & \\
\hline 428 & 0.03 & 6.09 & 0.76 & 155 & & & & & & & & & \\
\hline 432 & 0.00 & 6.18 & 0.00 & 157 & & & & & & & & & \\
\hline 433 & 0.00 & 6.18 & 0.00 & 157 & & & & & & & & & \\
\hline 434 & 0.00 & 6.18 & 0.00 & 157 & & & & & & & & & \\
\hline 435 & 0.00 & 6.18 & 0.00 & 157 & & & & & & & & & \\
\hline 436 & 0.00 & 6.18 & 0.00 & 157 & & & & & & & & & \\
\hline 437 & 0.00 & 6.18 & 0.00 & 157 & & & & & & & & & \\
\hline
\end{tabular}


Table 7. Daily and cumulative precipitation at the Montrose \#2 climate station, upper Elephant Skin Wash, and Candy Lane rain gages, southwestern

Colorado.-Continued

[in., inch; mm, millimeter; md, missing data due to equipment malfunction or vandalism; MA, Montrose \#2 climate station; ES, upper Elephant Skin Wash; CL, Candy Lane]

\begin{tabular}{|c|c|c|c|c|c|c|c|c|c|c|c|c|c|}
\hline \multirow[b]{2}{*}{$\begin{array}{c}\text { Julian day } \\
\text { since } 1 \\
\text { January } \\
2003\end{array}$} & \multicolumn{4}{|c|}{ Montrose \#2 climate station rain gage } & \multicolumn{4}{|c|}{ Upper Elephant Skin Wash rain gage } & \multicolumn{4}{|c|}{ Candy Lane rain gage } & \multirow[b]{2}{*}{ Remark } \\
\hline & $\begin{array}{l}\text { Precip- } \\
\text { itation } \\
\text { (in.) } \\
\end{array}$ & $\begin{array}{l}\text { Cumu- } \\
\text { lative } \\
\text { precip- } \\
\text { itation } \\
\text { (in.) }\end{array}$ & $\begin{array}{l}\text { Precip- } \\
\text { itation } \\
(\mathrm{mm})\end{array}$ & $\begin{array}{l}\text { Cumu- } \\
\text { lative } \\
\text { precip- } \\
\text { itation } \\
(\mathrm{mm})\end{array}$ & $\begin{array}{l}\text { Precip- } \\
\text { itation } \\
\text { (in.) } \\
\end{array}$ & $\begin{array}{l}\text { Cumu- } \\
\text { lative } \\
\text { precip- } \\
\text { itation } \\
\text { (in.) }\end{array}$ & $\begin{array}{l}\text { Precip- } \\
\text { itation } \\
(\mathrm{mm}) \\
\end{array}$ & $\begin{array}{l}\text { Cumu- } \\
\text { lative } \\
\text { precip- } \\
\text { itation } \\
(\mathrm{mm})\end{array}$ & $\begin{array}{l}\text { Precip- } \\
\text { itation } \\
\text { (in.) } \\
\end{array}$ & $\begin{array}{l}\text { Cumu- } \\
\text { lative } \\
\text { precip- } \\
\text { itation } \\
\text { (in.) }\end{array}$ & $\begin{array}{l}\text { Precip- } \\
\text { itation } \\
(\mathrm{mm}) \\
\end{array}$ & $\begin{array}{l}\text { Cumu- } \\
\text { lative } \\
\text { precip- } \\
\text { itation } \\
(\mathrm{mm})\end{array}$ & \\
\hline 438 & 0.00 & 6.18 & 0.00 & 157 & & & & & & & & & \\
\hline 439 & 0.00 & 6.18 & 0.00 & 157 & & & & & & & & & \\
\hline 440 & 0.00 & 6.18 & 0.00 & 157 & & & & & & & & & \\
\hline 441 & 0.00 & 6.18 & 0.00 & 157 & & & & & & & & & \\
\hline 443 & 0.00 & 6.18 & 0.00 & 157 & & & & & & & & & \\
\hline 444 & 0.00 & 6.18 & 0.00 & 157 & & & & & & & & & \\
\hline 445 & 0.00 & 6.18 & 0.00 & 157 & & & & & & & & & \\
\hline 446 & 0.00 & 6.18 & 0.00 & 157 & & & & & & & & & \\
\hline 447 & 0.00 & 6.18 & 0.00 & 157 & & & & & & & & & \\
\hline 448 & 0.00 & 6.18 & 0.00 & 157 & & & & & & & & & \\
\hline 449 & 0.00 & 6.18 & 0.00 & 157 & & & & & & & & & \\
\hline 450 & 0.00 & 6.18 & 0.00 & 157 & & & & & & & & & \\
\hline 451 & 0.00 & 6.18 & 0.00 & 157 & & & & & & & & & \\
\hline 452 & 0.00 & 6.18 & 0.00 & 157 & & & & & & & & & \\
\hline 454 & 0.00 & 6.18 & 0.00 & 157 & & & & & & & & & \\
\hline 455 & 0.00 & 6.18 & 0.00 & 157 & & & & & & & & & \\
\hline 456 & 0.00 & 6.18 & 0.00 & 157 & & & & & & & & & \\
\hline 457 & 0.00 & 6.18 & 0.00 & 157 & & & & & & & & & 1 April 2004 \\
\hline 458 & 0.00 & 6.18 & 0.00 & 157 & & & & & & & & & \\
\hline 459 & 0.00 & 6.18 & 0.00 & 157 & & & & & & & & & \\
\hline 460 & 0.05 & 6.23 & 1.27 & 158 & & & & & & & & & \\
\hline 461 & 0.90 & 7.13 & 22.86 & 181 & & & & & & & & & \\
\hline 462 & 0.02 & 7.15 & 0.51 & 182 & & & & & & & & & \\
\hline 463 & Trace & 7.15 & Trace & 182 & & & & & & & & & \\
\hline 464 & 0.42 & 7.57 & 10.67 & 192 & & & & & & & & & \\
\hline 465 & 0.24 & 7.81 & 6.10 & 198 & & & & & & & & & \\
\hline 466 & 0.10 & 7.91 & 2.54 & 201 & & & & & & & & & \\
\hline 467 & 0.15 & 8.06 & 3.81 & 205 & & & & & & & & & \\
\hline 468 & Trace & 8.07 & Trace & 205 & & & & & & & & & \\
\hline 469 & 0.00 & 8.07 & 0.00 & 205 & & & & & & & & & \\
\hline 470 & 0.00 & 8.07 & 0.00 & 205 & & & & & & & & & \\
\hline 471 & 0.00 & 8.07 & 0.00 & 205 & & & & & & & & & \\
\hline 472 & 0.00 & 8.07 & 0.00 & 205 & & & & & & & & & \\
\hline 473 & 0.00 & 8.07 & 0.00 & 205 & & & & & & & & & \\
\hline 474 & 0.04 & 8.11 & 1.02 & 206 & & & & & & & & & \\
\hline 477 & 0.00 & 8.11 & 0.00 & 206 & & & & & & & & & \\
\hline 478 & 0.02 & 8.13 & 0.51 & 206 & & & & & & & & & \\
\hline 479 & 0.38 & 8.51 & 9.65 & 216 & & & & & & & & & \\
\hline 480 & 0.02 & 8.53 & 0.51 & 217 & & & & & & & & & \\
\hline 481 & 0.02 & 8.55 & 0.51 & 217 & & & & & & & & & \\
\hline 482 & 0.00 & 8.55 & 0.00 & 217 & & & & & & & & & \\
\hline
\end{tabular}


Table 7. Daily and cumulative precipitation at the Montrose \#2 climate station, upper Elephant Skin Wash, and Candy Lane rain gages, southwestern Colorado.-Continued

[in., inch; mm, millimeter; md, missing data due to equipment malfunction or vandalism; MA, Montrose \#2 climate station; ES, upper Elephant Skin Wash; CL, Candy Lane]

\begin{tabular}{|c|c|c|c|c|c|c|c|c|c|c|c|c|c|}
\hline \multirow[b]{2}{*}{$\begin{array}{c}\text { Julian day } \\
\text { since } 1 \\
\text { January } \\
2003\end{array}$} & \multicolumn{4}{|c|}{ Montrose \#2 climate station rain gage } & \multicolumn{4}{|c|}{ Upper Elephant Skin Wash rain gage } & \multicolumn{4}{|c|}{ Candy Lane rain gage } & \multirow[b]{2}{*}{ Remark } \\
\hline & $\begin{array}{l}\text { Precip- } \\
\text { itation } \\
\text { (in.) } \\
\end{array}$ & $\begin{array}{l}\text { Cumu- } \\
\text { lative } \\
\text { precip- } \\
\text { itation } \\
\text { (in.) }\end{array}$ & $\begin{array}{l}\text { Precip- } \\
\text { itation } \\
(\mathrm{mm})\end{array}$ & $\begin{array}{l}\text { Cumu- } \\
\text { lative } \\
\text { precip- } \\
\text { itation } \\
(\mathrm{mm})\end{array}$ & $\begin{array}{l}\text { Precip- } \\
\text { itation } \\
\text { (in.) } \\
\end{array}$ & $\begin{array}{l}\text { Cumu- } \\
\text { lative } \\
\text { precip- } \\
\text { itation } \\
\text { (in.) }\end{array}$ & $\begin{array}{l}\text { Precip- } \\
\text { itation } \\
(\mathrm{mm})\end{array}$ & $\begin{array}{l}\text { Cumu- } \\
\text { lative } \\
\text { precip- } \\
\text { itation } \\
(\mathrm{mm})\end{array}$ & $\begin{array}{c}\text { Precip- } \\
\text { itation } \\
\text { (in.) } \\
\end{array}$ & $\begin{array}{l}\text { Cumu- } \\
\text { lative } \\
\text { precip- } \\
\text { itation } \\
\text { (in.) }\end{array}$ & $\begin{array}{l}\text { Precip- } \\
\text { itation } \\
(\mathrm{mm})\end{array}$ & $\begin{array}{l}\text { Cumu- } \\
\text { lative } \\
\text { precip- } \\
\text { itation } \\
(\mathrm{mm})\end{array}$ & \\
\hline 483 & 0.00 & 8.55 & 0.00 & 217 & & & & & & & & & \\
\hline 484 & 0.00 & 8.55 & 0.00 & 217 & & & & & & & & & \\
\hline 485 & 0.00 & 8.55 & 0.00 & 217 & & & & & & & & & \\
\hline 487 & 0.13 & 9.15 & 3.30 & 232 & & & & & & & & & 1 May 2004 \\
\hline 488 & 0.00 & 9.15 & 0.00 & 232 & & & & & & & & & \\
\hline 489 & 0.00 & 9.15 & 0.00 & 232 & & & & & & & & & \\
\hline 490 & 0.00 & 9.15 & 0.00 & 232 & & & & & & & & & \\
\hline 491 & 0.00 & 9.15 & 0.00 & 232 & & & & & & & & & \\
\hline 492 & 0.00 & 9.15 & 0.00 & 232 & & & & & & & & & \\
\hline 493 & 0.00 & 9.15 & 0.00 & 232 & & & & & & & & & \\
\hline 494 & 0.00 & 9.15 & 0.00 & 232 & & & & & & & & & \\
\hline 495 & 0.00 & 9.15 & 0.00 & 232 & & & & & & & & & \\
\hline 496 & 0.00 & 9.15 & 0.00 & 232 & & & & & & & & & \\
\hline 499 & 0.12 & 9.27 & 3.05 & 235 & & & & & & & & & \\
\hline 500 & 0.04 & 9.31 & 1.02 & 236 & & & & & & & & & \\
\hline 501 & 0.00 & 9.31 & 0.00 & 236 & & & & & & & & & \\
\hline 502 & 0.00 & 9.31 & 0.00 & 236 & & & & & & & & & \\
\hline 503 & 0.00 & 9.31 & 0.00 & 236 & & & & & & & & & \\
\hline 504 & 0.00 & 9.31 & 0.00 & 236 & & & & & & & & & \\
\hline 505 & 0.00 & 9.31 & 0.00 & 236 & & & & & & & & & \\
\hline 506 & 0.00 & 9.31 & 0.00 & 236 & & & & & & & & & \\
\hline 507 & 0.00 & 9.31 & 0.00 & 236 & & & & & & & & & \\
\hline 508 & 0.00 & 9.31 & 0.00 & 236 & & & & & & & & & \\
\hline 509 & 0.00 & 9.31 & 0.00 & 236 & & & & & & & & & \\
\hline 510 & 0.00 & 9.31 & 0.00 & 236 & & & & & & & & & \\
\hline 511 & 0.00 & 9.31 & 0.00 & 236 & & & & & & & & & \\
\hline 512 & 0.00 & 9.31 & 0.00 & 236 & & & & & & & & & \\
\hline 513 & 0.00 & 9.31 & 0.00 & 236 & & & & & & & & & \\
\hline 514 & 0.00 & 9.31 & 0.00 & 236 & & & & & & & & & \\
\hline 515 & 0.00 & 9.31 & 0.00 & 236 & & & & & & & & & \\
\hline 516 & 0.00 & 9.31 & 0.00 & 236 & & & & & & & & & \\
\hline 517 & 0.00 & 9.31 & 0.00 & 236 & & & & & & & & & \\
\hline 518 & 0.00 & 9.31 & 0.00 & 236 & & & & & & & & & 1 June 2004 \\
\hline 522 & 0.00 & 9.31 & 0.00 & 236 & & & & & & & & & \\
\hline 523 & 0.00 & 9.31 & 0.00 & 236 & & & & & & & & & \\
\hline 524 & 0.00 & 9.31 & 0.00 & 236 & & & & & & & & & \\
\hline 525 & 0.00 & 9.31 & 0.00 & 236 & & & & & & & & & \\
\hline 526 & 0.00 & 9.31 & 0.00 & 236 & & & & & & & & & \\
\hline 527 & 0.00 & 9.31 & 0.00 & 236 & & & & & & & & & \\
\hline
\end{tabular}


Table 7. Daily and cumulative precipitation at the Montrose \#2 climate station, upper Elephant Skin Wash, and Candy Lane rain gages, southwestern Colorado.-Continued

[in., inch; mm, millimeter; md, missing data due to equipment malfunction or vandalism; MA, Montrose \#2 climate station; ES, upper Elephant Skin Wash; CL, Candy Lane]

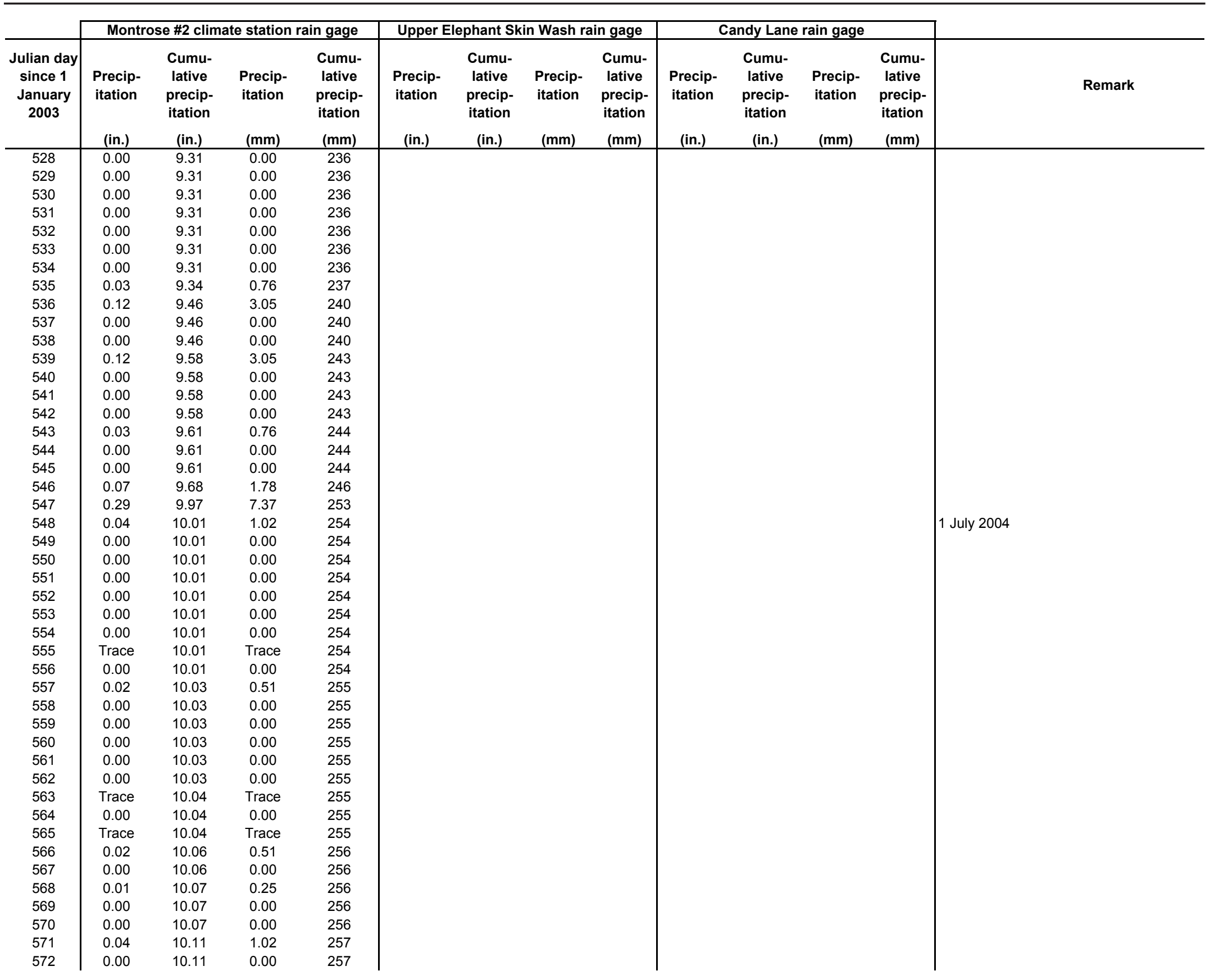


Table 7. Daily and cumulative precipitation at the Montrose \#2 climate station, upper Elephant Skin Wash, and Candy Lane rain gages, southwestern Colorado.-Continued

[in., inch; mm, millimeter; md, missing data due to equipment malfunction or vandalism; MA, Montrose \#2 climate station; ES, upper Elephant Skin Wash; CL, Candy Lane]

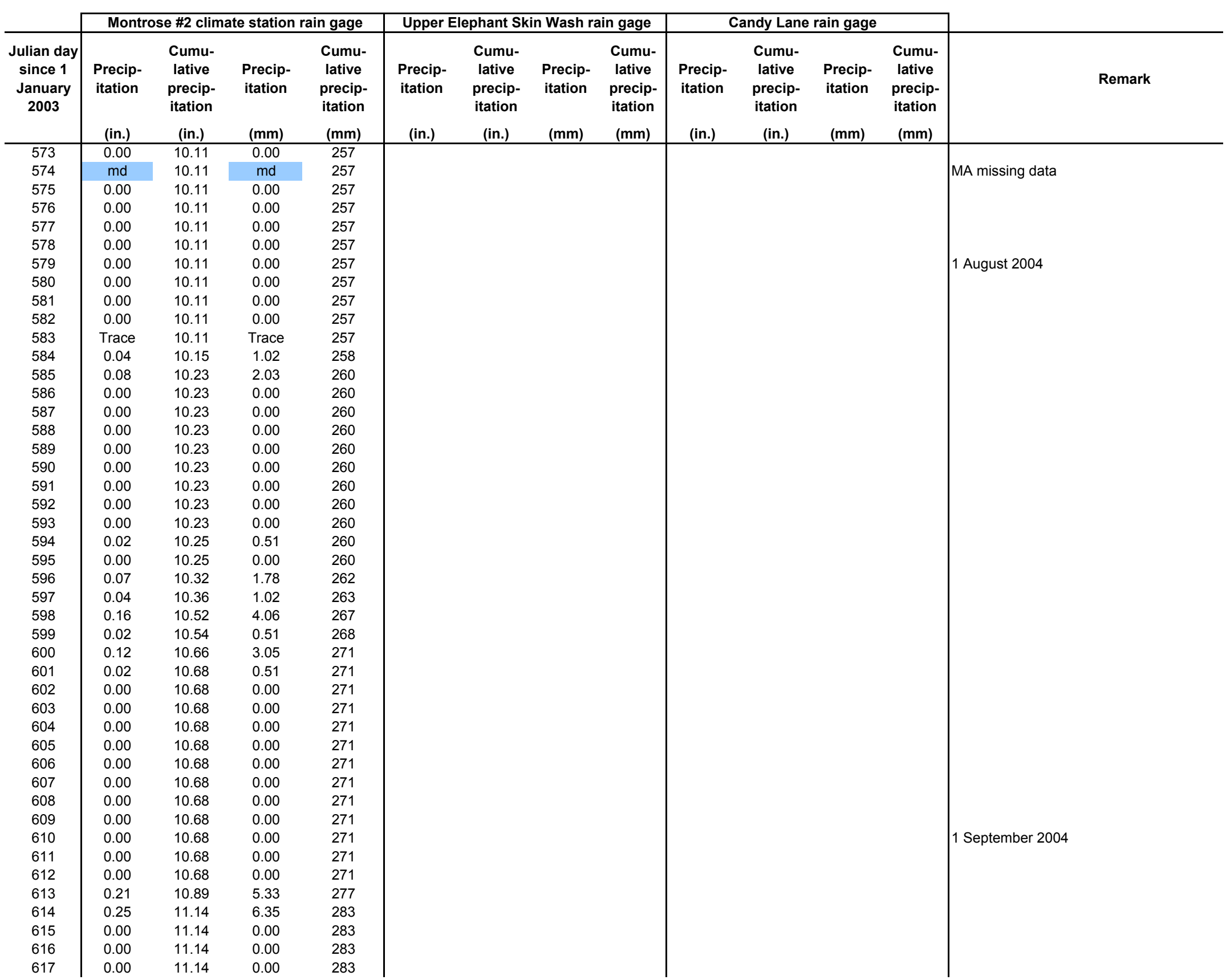


Table 7. Daily and cumulative precipitation at the Montrose \#2 climate station, upper Elephant Skin Wash, and Candy Lane rain gages, southwestern Colorado.-Continued

[in., inch; mm, millimeter; md, missing data due to equipment malfunction or vandalism; MA, Montrose \#2 climate station; ES, upper Elephant Skin Wash; CL, Candy Lane]

\begin{tabular}{|c|c|c|c|c|c|c|c|c|c|c|c|c|c|}
\hline \multirow[b]{2}{*}{$\begin{array}{c}\text { Julian day } \\
\text { since } 1 \\
\text { January } \\
2003\end{array}$} & \multicolumn{4}{|c|}{ Montrose \#2 climate station rain gage } & \multicolumn{4}{|c|}{ Upper Elephant Skin Wash rain gage } & \multicolumn{4}{|c|}{ Candy Lane rain gage } & \multirow[b]{2}{*}{ Remark } \\
\hline & $\begin{array}{l}\text { Precip- } \\
\text { itation } \\
\text { (in.) }\end{array}$ & $\begin{array}{l}\text { Cumu- } \\
\text { lative } \\
\text { precip- } \\
\text { itation } \\
\text { (in.) }\end{array}$ & $\begin{array}{l}\text { Precip- } \\
\text { itation } \\
(\mathrm{mm})\end{array}$ & $\begin{array}{l}\text { Cumu- } \\
\text { lative } \\
\text { precip- } \\
\text { itation } \\
(\mathrm{mm})\end{array}$ & $\begin{array}{l}\text { Precip- } \\
\text { itation } \\
\text { (in.) }\end{array}$ & $\begin{array}{l}\text { Cumu- } \\
\text { lative } \\
\text { precip- } \\
\text { itation } \\
\text { (in.) }\end{array}$ & $\begin{array}{l}\text { Precip- } \\
\text { itation } \\
(\mathrm{mm})\end{array}$ & $\begin{array}{l}\text { Cumu- } \\
\text { lative } \\
\text { precip- } \\
\text { itation } \\
(\mathrm{mm})\end{array}$ & $\begin{array}{l}\text { Precip- } \\
\text { itation } \\
\text { (in.) }\end{array}$ & $\begin{array}{l}\text { Cumu- } \\
\text { lative } \\
\text { precip- } \\
\text { itation } \\
\text { (in.) }\end{array}$ & $\begin{array}{l}\text { Precip- } \\
\text { itation } \\
(\mathrm{mm})\end{array}$ & $\begin{array}{l}\text { Cumu- } \\
\text { lative } \\
\text { precip- } \\
\text { itation } \\
(\mathrm{mm})\end{array}$ & \\
\hline 618 & 0.00 & 11.14 & 0.00 & 283 & & & & & & & & & \\
\hline 619 & 0.05 & 11.19 & 1.27 & 284 & & & & & & & & & \\
\hline 620 & 0.00 & 11.19 & 0.00 & 284 & & & & & & & & & \\
\hline 621 & 0.00 & 11.19 & 0.00 & 284 & & & & & & & & & \\
\hline 622 & 0.00 & 11.19 & 0.00 & 284 & & & & & & & & & \\
\hline 623 & 0.00 & 11.19 & 0.00 & 284 & & & & & & & & & \\
\hline 624 & 0.00 & 11.19 & 0.00 & 284 & & & & & & & & & \\
\hline 625 & 0.00 & 11.19 & 0.00 & 284 & & & & & & & & & \\
\hline 626 & 0.00 & 11.19 & 0.00 & 284 & & & & & & & & & \\
\hline 627 & 0.00 & 11.19 & 0.00 & 284 & & & & & & & & & \\
\hline 628 & 0.07 & 11.26 & 1.78 & 286 & & & & & & & & & \\
\hline 629 & 0.42 & 11.68 & 10.67 & 297 & & & & & & & & & \\
\hline 630 & 0.65 & 12.33 & 16.51 & 313 & & & & & & & & & \\
\hline 631 & 0.58 & 12.91 & 14.73 & 328 & & & & & & & & & \\
\hline 632 & 0.04 & 12.95 & 1.02 & 329 & & & & & & & & & \\
\hline 633 & 0.00 & 12.95 & 0.00 & 329 & & & & & & & & & \\
\hline 634 & 0.00 & 12.95 & 0.00 & 329 & & & & & & & & & \\
\hline 635 & 0.00 & 12.95 & 0.00 & 329 & & & & & & & & & \\
\hline 636 & 0.00 & 12.95 & 0.00 & 329 & & & & & & & & & \\
\hline 637 & 0.00 & 12.95 & 0.00 & 329 & & & & & & & & & \\
\hline 638 & 0.04 & 12.99 & 1.02 & 330 & & & & & & & & & \\
\hline 639 & 0.23 & 13.22 & 5.84 & 336 & & & & & & & & & \\
\hline 640 & 0.23 & 13.45 & 5.84 & 342 & & & & & & & & & 1 October 2004 \\
\hline 641 & 0.00 & 13.45 & 0.00 & 342 & & & & & & & & & \\
\hline 642 & 0.00 & 13.45 & 0.00 & 342 & & & & & & & & & \\
\hline 643 & 0.00 & 13.45 & 0.00 & 342 & & & & & & & & & \\
\hline 644 & 0.05 & 13.50 & 1.27 & 343 & & & & & & & & & \\
\hline 645 & 0.07 & 13.57 & 1.78 & 345 & & & & & & & & & \\
\hline 646 & 0.02 & 13.59 & 0.51 & 345 & & & & & & & & & \\
\hline 647 & 0.00 & 13.59 & 0.00 & 345 & & & & & & & & & \\
\hline 648 & 0.00 & 13.59 & 0.00 & 345 & & & & & & & & & \\
\hline 649 & 0.00 & 13.59 & 0.00 & 345 & & & & & & & & & \\
\hline 650 & 0.00 & 13.59 & 0.00 & 345 & & & & & & & & & \\
\hline 651 & 0.00 & 13.59 & 0.00 & 345 & & & & & & & & & \\
\hline 652 & 0.23 & 13.82 & 5.84 & 351 & & & & & & & & & \\
\hline 653 & 0.00 & 13.82 & 0.00 & 351 & & & & & & & & & \\
\hline 654 & 0.00 & 13.82 & 0.00 & 351 & & & & & & & & & \\
\hline 655 & 0.00 & 13.82 & 0.00 & 351 & & & & & & & & & \\
\hline 656 & 0.00 & 13.82 & 0.00 & 351 & & & & & & & & & \\
\hline 657 & 0.02 & 13.84 & 0.51 & 352 & & & & & & & & & \\
\hline 658 & 0.00 & 13.84 & 0.00 & 352 & 0.00 & 0.00 & 0.00 & 0 & 0.00 & 0.00 & 0.00 & 0 & 19 October 2004, ES and CL rain gages installed \\
\hline 659 & 0.00 & 13.84 & 0.00 & 352 & 0.00 & 0.00 & 0.00 & 0 & 0.00 & 0.00 & 0.00 & 0 & \\
\hline 660 & 0.00 & 13.84 & 0.00 & 352 & 0.00 & 0.00 & 0.00 & 0 & 0.04 & 0.04 & 1.02 & 1 & \\
\hline 661 & 0.00 & 13.84 & 0.00 & 352 & 0.00 & 0.00 & 0.00 & 0 & 0.02 & 0.06 & 0.51 & 2 & \\
\hline 662 & 0.02 & 13.86 & 0.51 & 352 & 0.00 & 0.00 & 0.00 & 0 & 0.00 & 0.06 & 0.00 & 2 & \\
\hline
\end{tabular}


Table 7. Daily and cumulative precipitation at the Montrose \#2 climate station, upper Elephant Skin Wash, and Candy Lane rain gages, southwestern Colorado.-Continued

[in., inch; mm, millimeter; md, missing data due to equipment malfunction or vandalism; MA, Montrose \#2 climate station; ES, upper Elephant Skin Wash; CL, Candy Lane]

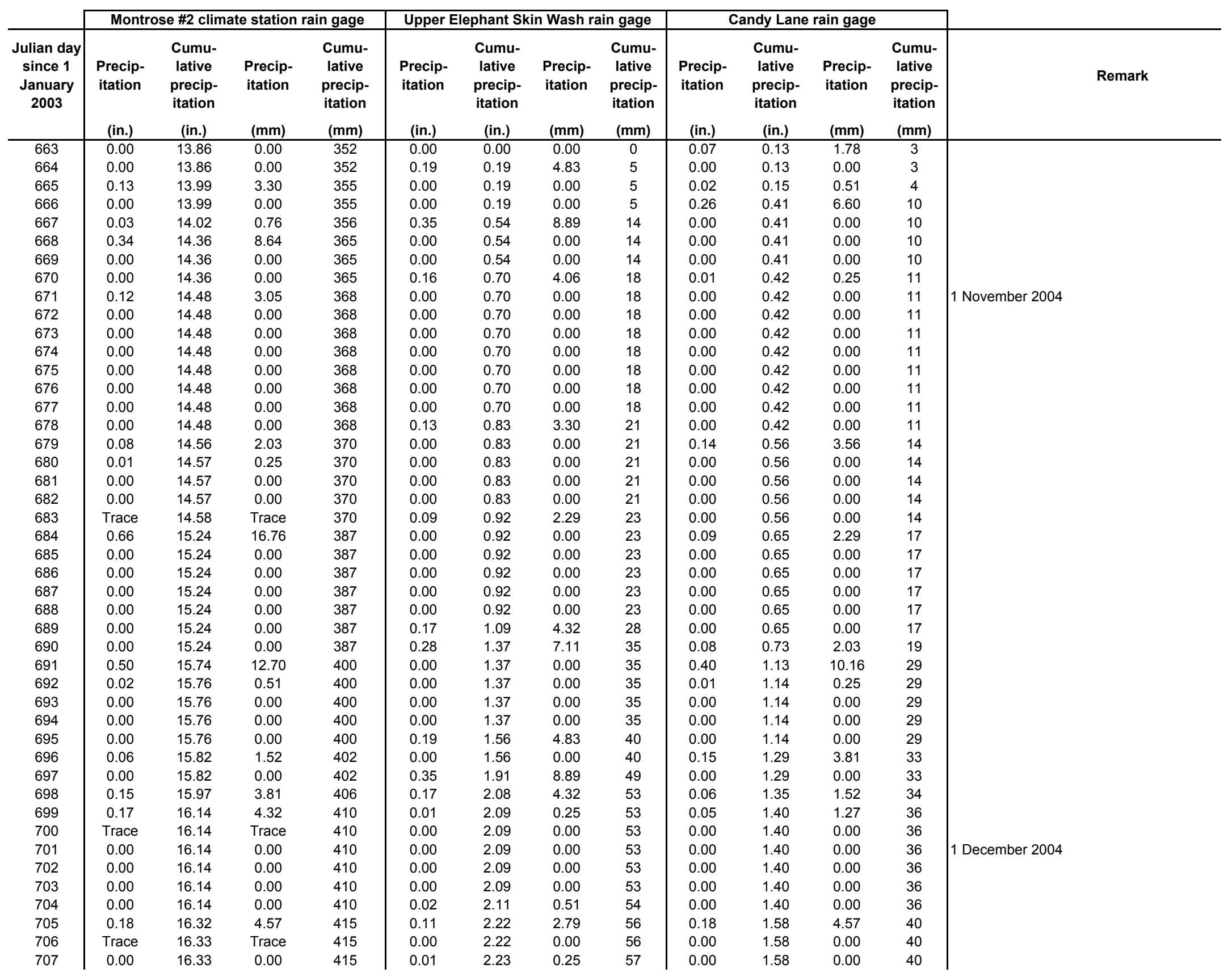


Table 7. Daily and cumulative precipitation at the Montrose \#2 climate station, upper Elephant Skin Wash, and Candy Lane rain gages, southwestern Colorado.-Continued

[in., inch; mm, millimeter; md, missing data due to equipment malfunction or vandalism; MA, Montrose \#2 climate station; ES, upper Elephant Skin Wash; CL, Candy Lane]

\begin{tabular}{|c|c|c|c|c|c|c|c|c|c|c|c|c|c|}
\hline \multirow[b]{2}{*}{$\begin{array}{c}\text { Julian day } \\
\text { since } 1 \\
\text { January } \\
2003\end{array}$} & \multicolumn{4}{|c|}{ Montrose \#2 climate station rain gage } & \multicolumn{4}{|c|}{ Upper Elephant Skin Wash rain gage } & \multicolumn{4}{|c|}{ Candy Lane rain gage } & \multirow[b]{2}{*}{ Remark } \\
\hline & $\begin{array}{l}\text { Precip- } \\
\text { itation } \\
\text { (in.) } \\
\end{array}$ & $\begin{array}{l}\text { Cumu- } \\
\text { lative } \\
\text { precip- } \\
\text { itation } \\
\text { (in.) }\end{array}$ & $\begin{array}{l}\text { Precip- } \\
\text { itation } \\
(\mathrm{mm}) \\
\end{array}$ & $\begin{array}{l}\text { Cumu- } \\
\text { lative } \\
\text { precip- } \\
\text { itation } \\
(\mathrm{mm})\end{array}$ & $\begin{array}{l}\text { Precip- } \\
\text { itation } \\
\text { (in.) } \\
\end{array}$ & $\begin{array}{l}\text { Cumu- } \\
\text { lative } \\
\text { precip- } \\
\text { itation } \\
\text { (in.) }\end{array}$ & $\begin{array}{l}\text { Precip- } \\
\text { itation } \\
(\mathrm{mm}) \\
\end{array}$ & $\begin{array}{l}\text { Cumu- } \\
\text { lative } \\
\text { precip- } \\
\text { itation } \\
(\mathrm{mm})\end{array}$ & $\begin{array}{l}\text { Precip- } \\
\text { itation } \\
\text { (in.) } \\
\end{array}$ & $\begin{array}{l}\text { Cumu- } \\
\text { lative } \\
\text { precip- } \\
\text { itation } \\
\text { (in.) }\end{array}$ & $\begin{array}{l}\text { Precip- } \\
\text { itation } \\
(\mathrm{mm})\end{array}$ & $\begin{array}{l}\text { Cumu- } \\
\text { lative } \\
\text { precip- } \\
\text { itation } \\
(\mathrm{mm})\end{array}$ & \\
\hline 708 & 0.00 & 16.33 & 0.00 & 415 & 0.01 & 2.24 & 0.25 & 57 & 0.03 & 1.61 & 0.76 & 41 & \\
\hline 709 & Trace & 16.33 & Trace & 415 & 0.00 & 2.24 & 0.00 & 57 & 0.00 & 1.61 & 0.00 & 41 & \\
\hline 710 & 0.00 & 16.33 & 0.00 & 415 & 0.00 & 2.24 & 0.00 & 57 & 0.00 & 1.61 & 0.00 & 41 & \\
\hline 712 & 0.00 & 16.33 & 0.00 & 415 & 0.00 & 2.24 & 0.00 & 57 & 0.00 & 1.61 & 0.00 & 41 & \\
\hline 713 & 0.00 & 16.33 & 0.00 & 415 & 0.00 & 2.24 & 0.00 & 57 & 0.00 & 1.61 & 0.00 & 41 & \\
\hline 714 & 0.00 & 16.33 & 0.00 & 415 & 0.00 & 2.24 & 0.00 & 57 & 0.00 & 1.61 & 0.00 & 41 & \\
\hline 715 & 0.00 & 16.33 & 0.00 & 415 & 0.00 & 2.24 & 0.00 & 57 & 0.00 & 1.61 & 0.00 & 41 & \\
\hline 716 & 0.00 & 16.33 & 0.00 & 415 & 0.00 & 2.24 & 0.00 & 57 & 0.00 & 1.61 & 0.00 & 41 & \\
\hline 717 & 0.00 & 16.33 & 0.00 & 415 & 0.00 & 2.24 & 0.00 & 57 & 0.00 & 1.61 & 0.00 & 41 & \\
\hline 718 & 0.00 & 16.33 & 0.00 & 415 & 0.00 & 2.24 & 0.00 & 57 & 0.00 & 1.61 & 0.00 & 41 & \\
\hline 719 & 0.00 & 16.33 & 0.00 & 415 & 0.00 & 2.24 & 0.00 & 57 & 0.00 & 1.61 & 0.00 & 41 & \\
\hline 720 & 0.00 & 16.33 & 0.00 & 415 & 0.00 & 2.24 & 0.00 & 57 & 0.00 & 1.61 & 0.00 & 41 & \\
\hline 721 & 0.00 & 16.33 & 0.00 & 415 & 0.00 & 2.24 & 0.00 & 57 & 0.00 & 1.61 & 0.00 & 41 & \\
\hline 724 & 0.00 & 16.34 & 0.00 & 415 & 0.00 & 2.26 & 0.00 & 57 & 0.00 & 1.61 & 0.00 & 41 & \\
\hline 725 & 0.00 & 16.34 & 0.00 & 415 & 0.00 & 2.26 & 0.00 & 57 & 0.00 & 1.61 & 0.00 & 41 & \\
\hline 726 & 0.00 & 16.34 & 0.00 & 415 & 0.00 & 2.26 & 0.00 & 57 & 0.00 & 1.61 & 0.00 & 41 & \\
\hline 727 & 0.00 & 16.34 & 0.00 & 415 & 0.00 & 2.26 & 0.00 & 57 & 0.00 & 1.61 & 0.00 & 41 & \\
\hline 728 & 0.00 & 16.34 & 0.00 & 415 & 0.20 & 2.46 & 5.08 & 62 & 0.00 & 1.61 & 0.00 & 41 & \\
\hline 729 & 0.03 & 16.37 & 0.76 & 416 & 0.00 & 2.46 & 0.00 & 62 & 0.31 & 1.92 & 7.87 & 49 & \\
\hline 730 & 0.15 & 16.52 & 3.81 & 420 & 0.00 & 2.46 & 0.00 & 62 & 0.00 & 1.92 & 0.00 & 49 & \\
\hline 731 & 0.00 & 16.52 & 0.00 & 420 & 0.00 & 2.46 & 0.00 & 62 & 0.00 & 1.92 & 0.00 & 49 & \\
\hline 732 & 0.00 & 16.52 & 0.00 & 420 & 0.00 & 2.46 & 0.00 & 62 & 0.00 & 1.92 & 0.00 & 49 & 1 January 2005 \\
\hline 733 & 0.00 & 16.52 & 0.00 & 420 & 0.00 & 2.46 & 0.00 & 62 & 0.00 & 1.92 & 0.00 & 49 & \\
\hline 734 & 0.00 & 16.52 & 0.00 & 420 & 0.16 & 2.62 & 4.06 & 67 & 0.00 & 1.92 & 0.00 & 49 & \\
\hline 735 & 0.03 & 16.55 & 0.76 & 420 & 0.01 & 2.63 & 0.25 & 67 & 0.19 & 2.11 & 4.83 & 54 & \\
\hline 736 & 0.14 & 16.69 & 3.56 & 424 & 0.01 & 2.64 & 0.25 & 67 & 0.01 & 2.12 & 0.25 & 54 & \\
\hline 737 & Trace & 16.69 & Trace & 424 & 0.00 & 2.64 & 0.00 & 67 & 0.00 & 2.12 & 0.00 & 54 & \\
\hline 738 & 0.00 & 16.69 & 0.00 & 424 & 0.00 & 2.64 & 0.00 & 67 & 0.00 & 2.12 & 0.00 & 54 & \\
\hline 739 & 0.00 & 16.69 & 0.00 & 424 & 0.00 & 2.64 & 0.00 & 67 & 0.00 & 2.12 & 0.00 & 54 & \\
\hline 740 & 0.00 & 16.69 & 0.00 & 424 & 0.00 & 2.64 & 0.00 & 67 & 0.00 & 2.12 & 0.00 & 54 & \\
\hline 741 & 0.02 & 16.71 & 0.51 & 425 & 0.01 & 2.65 & 0.25 & 67 & 0.00 & 2.12 & 0.00 & 54 & \\
\hline 742 & 0.01 & 16.72 & 0.25 & 425 & 0.18 & 2.83 & 4.57 & 72 & 0.12 & 2.24 & 3.05 & 57 & \\
\hline 743 & 0.15 & 16.87 & 3.81 & 429 & 0.12 & 2.95 & 3.05 & 75 & 0.15 & 2.39 & 3.81 & 61 & \\
\hline 746 & 0.00 & 16.87 & 0.00 & 429 & 0.00 & 2.95 & 0.00 & 75 & 0.00 & 2.39 & 0.00 & 61 & \\
\hline 747 & 0.00 & 16.87 & 0.00 & 429 & 0.00 & 2.95 & 0.00 & 75 & 0.00 & 2.39 & 0.00 & 61 & \\
\hline 748 & 0.00 & 16.87 & 0.00 & 429 & 0.00 & 2.95 & 0.00 & 75 & 0.00 & 2.39 & 0.00 & 61 & \\
\hline 749 & 0.00 & 16.87 & 0.00 & 429 & 0.00 & 2.95 & 0.00 & 75 & 0.00 & 2.39 & 0.00 & 61 & \\
\hline 750 & 0.00 & 16.87 & 0.00 & 429 & 0.00 & 2.95 & 0.00 & 75 & 0.00 & 2.39 & 0.00 & 61 & \\
\hline 751 & 0.00 & 16.87 & 0.00 & 429 & 0.00 & 2.95 & 0.00 & 75 & 0.00 & 2.39 & 0.00 & 61 & \\
\hline 752 & 0.00 & 16.87 & 0.00 & 429 & 0.00 & 2.95 & 0.00 & 75 & 0.00 & 2.39 & 0.00 & 61 & \\
\hline
\end{tabular}


Table 7. Daily and cumulative precipitation at the Montrose \#2 climate station, upper Elephant Skin Wash, and Candy Lane rain gages, southwestern Colorado.-Continued

[in., inch; mm, millimeter; md, missing data due to equipment malfunction or vandalism; MA, Montrose \#2 climate station; ES, upper Elephant Skin Wash; CL, Candy Lane]

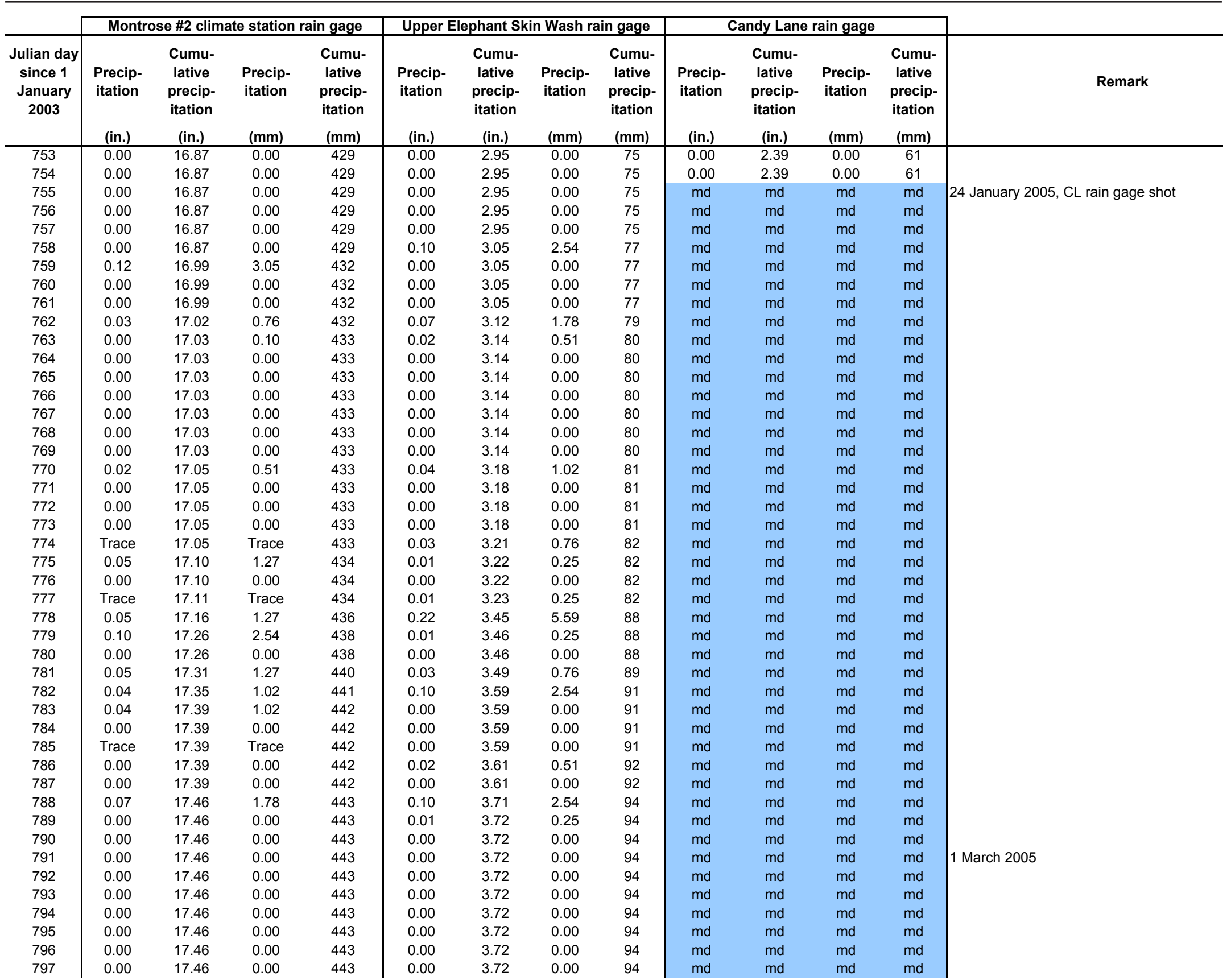


Table 7. Daily and cumulative precipitation at the Montrose \#2 climate station, upper Elephant Skin Wash, and Candy Lane rain gages, southwestern Colorado.-Continued

[in., inch; mm, millimeter; md, missing data due to equipment malfunction or vandalism; MA, Montrose \#2 climate station; ES, upper Elephant Skin Wash; CL, Candy Lane]

\begin{tabular}{|c|c|c|c|c|c|c|c|c|c|c|c|c|c|}
\hline \multirow[b]{2}{*}{$\begin{array}{c}\text { Julian day } \\
\text { since } 1 \\
\text { January } \\
2003\end{array}$} & \multicolumn{4}{|c|}{ Montrose \#2 climate station rain gage } & \multicolumn{4}{|c|}{ Upper Elephant Skin Wash rain gage } & \multicolumn{4}{|c|}{ Candy Lane rain gage } & \multirow[b]{2}{*}{ Remark } \\
\hline & $\begin{array}{l}\text { Precip- } \\
\text { itation } \\
\text { (in.) }\end{array}$ & $\begin{array}{l}\text { Cumu- } \\
\text { lative } \\
\text { precip- } \\
\text { itation } \\
\text { (in.) }\end{array}$ & $\begin{array}{l}\text { Precip- } \\
\text { itation } \\
(\mathrm{mm})\end{array}$ & $\begin{array}{l}\text { Cumu- } \\
\text { lative } \\
\text { precip- } \\
\text { itation } \\
(\mathrm{mm})\end{array}$ & $\begin{array}{l}\text { Precip- } \\
\text { itation } \\
\text { (in.) }\end{array}$ & $\begin{array}{l}\text { Cumu- } \\
\text { lative } \\
\text { precip- } \\
\text { itation } \\
\text { (in.) }\end{array}$ & $\begin{array}{l}\text { Precip- } \\
\text { itation } \\
(\mathrm{mm})\end{array}$ & $\begin{array}{c}\text { Cumu- } \\
\text { lative } \\
\text { precip- } \\
\text { itation } \\
(\mathrm{mm})\end{array}$ & $\begin{array}{l}\text { Precip- } \\
\text { itation } \\
\text { (in.) }\end{array}$ & $\begin{array}{l}\text { Cumu- } \\
\text { lative } \\
\text { precip- } \\
\text { itation } \\
\text { (in.) }\end{array}$ & $\begin{array}{l}\text { Precip- } \\
\text { itation } \\
(\mathrm{mm})\end{array}$ & $\begin{array}{l}\text { Cumu- } \\
\text { lative } \\
\text { precip- } \\
\text { itation } \\
(\mathrm{mm})\end{array}$ & \\
\hline 798 & 0.00 & 17.46 & 0.00 & 443 & 0.00 & 3.72 & 0.00 & 94 & $\mathrm{md}$ & $\mathrm{md}$ & $\mathrm{md}$ & md & \\
\hline 799 & 0.00 & 17.46 & 0.00 & 443 & 0.00 & 3.72 & 0.00 & 94 & $\mathrm{md}$ & $\mathrm{md}$ & $\mathrm{md}$ & $\mathrm{md}$ & \\
\hline 800 & 0.00 & 17.46 & 0.00 & 443 & 0.00 & 3.72 & 0.00 & 94 & $\mathrm{md}$ & $\mathrm{md}$ & $\mathrm{md}$ & $\mathrm{md}$ & \\
\hline 801 & 0.00 & 17.46 & 0.00 & 443 & 0.00 & 3.72 & 0.00 & 94 & $\mathrm{md}$ & $\mathrm{md}$ & $\mathrm{md}$ & $\mathrm{md}$ & \\
\hline 802 & 0.00 & 17.46 & 0.00 & 443 & 0.00 & 3.72 & 0.00 & 94 & $\mathrm{md}$ & $\mathrm{md}$ & $\mathrm{md}$ & $\mathrm{md}$ & \\
\hline 803 & 0.00 & 17.46 & 0.00 & 443 & 0.00 & 3.72 & 0.00 & 94 & $\mathrm{md}$ & $\mathrm{md}$ & $\mathrm{md}$ & $\mathrm{md}$ & \\
\hline 804 & 0.00 & 17.46 & 0.00 & 443 & 0.00 & 3.72 & 0.00 & 94 & $\mathrm{md}$ & $\mathrm{md}$ & $\mathrm{md}$ & $\mathrm{md}$ & \\
\hline 805 & 0.00 & 17.46 & 0.00 & 443 & 0.00 & 3.72 & 0.00 & 94 & $\mathrm{md}$ & $\mathrm{md}$ & $\mathrm{md}$ & md & \\
\hline 806 & 0.00 & 17.46 & 0.00 & 443 & 0.00 & 3.72 & 0.00 & 94 & $\mathrm{md}$ & $\mathrm{md}$ & $\mathrm{md}$ & $\mathrm{md}$ & \\
\hline 807 & 0.00 & 17.46 & 0.00 & 443 & 0.00 & 3.72 & 0.00 & 94 & $\mathrm{md}$ & $\mathrm{md}$ & $\mathrm{md}$ & $\mathrm{md}$ & \\
\hline 808 & 0.00 & 17.46 & 0.00 & 443 & 0.00 & 3.72 & 0.00 & 94 & $\mathrm{md}$ & $\mathrm{md}$ & $\mathrm{md}$ & md & \\
\hline 809 & 0.00 & 17.46 & 0.00 & 443 & 0.00 & 3.72 & 0.00 & 94 & $\mathrm{md}$ & $\mathrm{md}$ & $\mathrm{md}$ & $\mathrm{md}$ & \\
\hline 810 & Trace & 17.46 & Trace & 444 & 0.03 & 3.75 & 0.76 & 95 & $\mathrm{md}$ & $\mathrm{md}$ & $\mathrm{md}$ & md & \\
\hline 811 & 0.05 & 17.51 & 1.27 & 445 & 0.15 & 3.90 & 3.81 & 99 & $\mathrm{md}$ & $\mathrm{md}$ & $\mathrm{md}$ & $\mathrm{md}$ & \\
\hline 812 & 0.00 & 17.51 & 0.00 & 445 & 0.00 & 3.90 & 0.00 & 99 & $\mathrm{md}$ & md & md & md & Equate $C L$ cumulative precpitation to $\mathrm{ES}$ \\
\hline 813 & 0.00 & 17.51 & 0.00 & 445 & 0.10 & 4.00 & 2.54 & 102 & 0.00 & 3.90 & 0.00 & 99 & 23 March $2005, \mathrm{CL}$ rain gage relocated \\
\hline 814 & 0.03 & 17.54 & 0.76 & 446 & 0.00 & 4.00 & 0.00 & 102 & 0.22 & 4.12 & 5.59 & 105 & \\
\hline 815 & 0.04 & 17.58 & 1.02 & 447 & 0.05 & 4.05 & 1.27 & 103 & 0.03 & 4.15 & 0.76 & 105 & \\
\hline 816 & 0.05 & 17.63 & 1.27 & 448 & 0.00 & 4.05 & 0.00 & 103 & 0.00 & 4.15 & 0.00 & 105 & \\
\hline 817 & 0.00 & 17.63 & 0.00 & 448 & 0.00 & 4.05 & 0.00 & 103 & 0.00 & 4.15 & 0.00 & 105 & \\
\hline 818 & 0.00 & 17.63 & 0.00 & 448 & 0.00 & 4.05 & 0.00 & 103 & 0.00 & 4.15 & 0.00 & 105 & \\
\hline 819 & 0.00 & 17.63 & 0.00 & 448 & 0.41 & 4.46 & 10.41 & 113 & 0.08 & 4.23 & 2.03 & 107 & \\
\hline 820 & 0.27 & 17.90 & 6.86 & 455 & 0.01 & 4.47 & 0.25 & 114 & 0.10 & 4.33 & 2.54 & 110 & \\
\hline 821 & 0.06 & 17.96 & 1.52 & 456 & 0.00 & 4.47 & 0.00 & 114 & 0.00 & 4.33 & 0.00 & 110 & \\
\hline 822 & 0.00 & 17.96 & 0.00 & 456 & 0.00 & 4.47 & 0.00 & 114 & 0.00 & 4.33 & 0.00 & 110 & 1 April 2005 \\
\hline 823 & 0.00 & 17.96 & 0.00 & 456 & 0.00 & 4.47 & 0.00 & 114 & 0.00 & 4.33 & 0.00 & 110 & \\
\hline 824 & 0.00 & 17.96 & 0.00 & 456 & 0.00 & 4.47 & 0.00 & 114 & 0.00 & 4.33 & 0.00 & 110 & \\
\hline 825 & 0.00 & 17.96 & 0.00 & 456 & 0.00 & 4.47 & 0.00 & 114 & 0.00 & 4.33 & 0.00 & 110 & \\
\hline 826 & 0.00 & 17.96 & 0.00 & 456 & 0.07 & 4.54 & 1.78 & 115 & 0.00 & 4.33 & 0.00 & 110 & \\
\hline 827 & 0.00 & 17.96 & 0.00 & 456 & 0.00 & 4.54 & 0.00 & 115 & 0.00 & 4.33 & 0.00 & 110 & \\
\hline 828 & 0.00 & 17.96 & 0.00 & 456 & 0.00 & 4.54 & 0.00 & 115 & 0.00 & 4.33 & 0.00 & 110 & \\
\hline 829 & 0.00 & 17.96 & 0.00 & 456 & 0.00 & 4.54 & 0.00 & 115 & 0.00 & 4.33 & 0.00 & 110 & \\
\hline 830 & 0.00 & 17.96 & 0.00 & 456 & 0.00 & 4.54 & 0.00 & 115 & 0.00 & 4.33 & 0.00 & 110 & \\
\hline 831 & 0.10 & 18.06 & 2.54 & 459 & 0.06 & 4.60 & 1.52 & 117 & 0.00 & 4.33 & 0.00 & 110 & \\
\hline 832 & Trace & 18.07 & Trace & 459 & 0.00 & 4.60 & 0.00 & 117 & 0.00 & 4.33 & 0.00 & 110 & \\
\hline 833 & 0.00 & 18.07 & 0.00 & 459 & 0.00 & 4.60 & 0.00 & 117 & 0.00 & 4.33 & 0.00 & 110 & \\
\hline 834 & 0.00 & 18.07 & 0.00 & 459 & 0.00 & 4.60 & 0.00 & 117 & 0.00 & 4.33 & 0.00 & 110 & \\
\hline 835 & 0.00 & 18.07 & 0.00 & 459 & 0.00 & 4.60 & 0.00 & 117 & 0.00 & 4.33 & 0.00 & 110 & \\
\hline 836 & 0.00 & 18.07 & 0.00 & 459 & 0.00 & 4.60 & 0.00 & 117 & 0.00 & 4.33 & 0.00 & 110 & \\
\hline 837 & 0.00 & 18.07 & 0.00 & 459 & 0.00 & 4.60 & 0.00 & 117 & 0.00 & 4.33 & 0.00 & 110 & \\
\hline 838 & 0.00 & 18.07 & 0.00 & 459 & 0.00 & 4.60 & 0.00 & 117 & 0.12 & 4.45 & 3.05 & 113 & \\
\hline 839 & 0.00 & 18.07 & 0.00 & 459 & 0.00 & 4.60 & 0.00 & 117 & 0.00 & 4.45 & 0.00 & 113 & \\
\hline 840 & 0.00 & 18.07 & 0.00 & 459 & 0.00 & 4.60 & 0.00 & 117 & 0.00 & 4.45 & 0.00 & 113 & \\
\hline 841 & 0.00 & 18.07 & 0.00 & 459 & 0.11 & 4.71 & 2.79 & 120 & 0.05 & 4.50 & 1.27 & 114 & \\
\hline 842 & 0.09 & 18.16 & 2.29 & 461 & 0.00 & 4.71 & 0.00 & 120 & 0.00 & 4.50 & 0.00 & 114 & \\
\hline
\end{tabular}


Table 7. Daily and cumulative precipitation at the Montrose \#2 climate station, upper Elephant Skin Wash, and Candy Lane rain gages, southwestern Colorado.-Continued

[in., inch; mm, millimeter; md, missing data due to equipment malfunction or vandalism; MA, Montrose \#2 climate station; ES, upper Elephant Skin Wash; CL, Candy Lane]

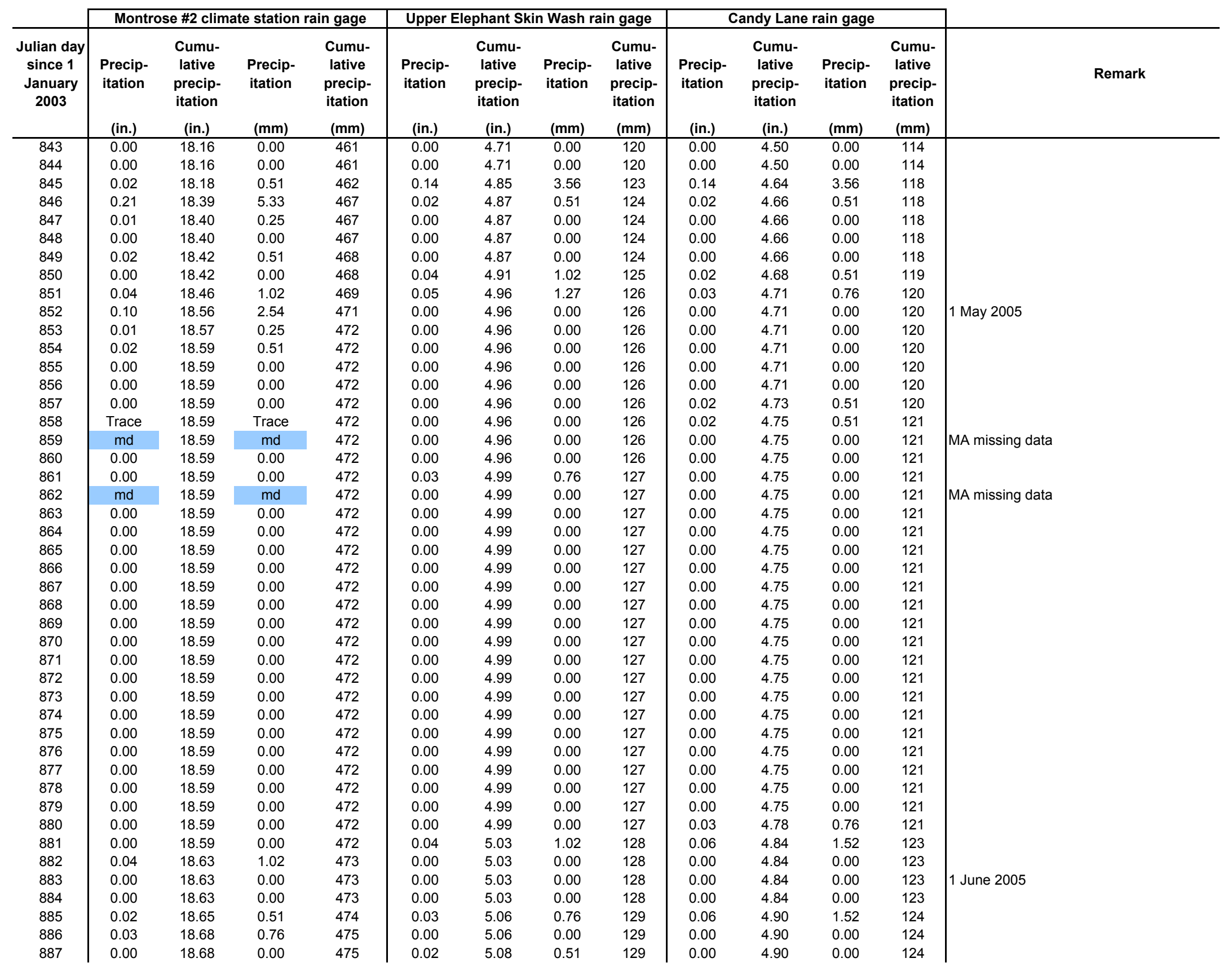


Table 7. Daily and cumulative precipitation at the Montrose \#2 climate station, upper Elephant Skin Wash, and Candy Lane rain gages, southwestern Colorado.-Continued

[in., inch; mm, millimeter; md, missing data due to equipment malfunction or vandalism; MA, Montrose \#2 climate station; ES, upper Elephant Skin Wash; CL, Candy Lane]

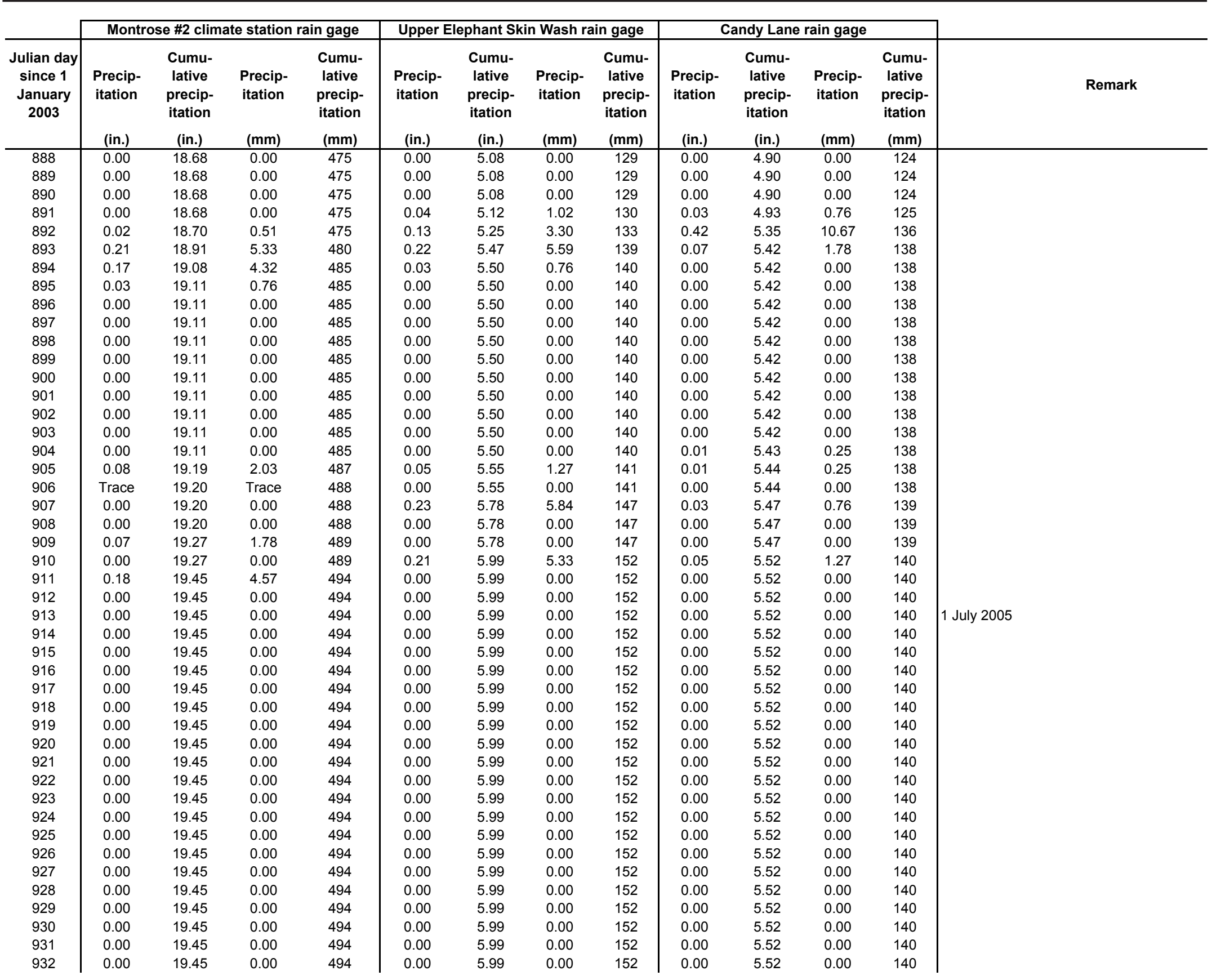


Table 7. Daily and cumulative precipitation at the Montrose \#2 climate station, upper Elephant Skin Wash, and Candy Lane rain gages, southwestern Colorado.-Continued

[in., inch; mm, millimeter; md, missing data due to equipment malfunction or vandalism; MA, Montrose \#2 climate station; ES, upper Elephant Skin Wash; CL, Candy Lane]

\begin{tabular}{|c|c|c|c|c|c|c|c|c|c|c|c|c|c|}
\hline \multirow[b]{2}{*}{$\begin{array}{c}\text { Julian day } \\
\text { since } 1 \\
\text { January } \\
2003\end{array}$} & \multicolumn{4}{|c|}{ Montrose \#2 climate station rain gage } & \multicolumn{4}{|c|}{ Upper Elephant Skin Wash rain gage } & \multicolumn{4}{|c|}{ Candy Lane rain gage } & \multirow[b]{2}{*}{ Remar } \\
\hline & $\begin{array}{c}\text { Precip- } \\
\text { itation } \\
\text { (in.) } \\
\end{array}$ & $\begin{array}{c}\text { Cumu- } \\
\text { lative } \\
\text { precip- } \\
\text { itation } \\
\text { (in.) }\end{array}$ & $\begin{array}{l}\text { Precip- } \\
\text { itation } \\
(\mathrm{mm})\end{array}$ & $\begin{array}{c}\text { Cumu- } \\
\text { lative } \\
\text { precip- } \\
\text { itation } \\
(\mathrm{mm})\end{array}$ & $\begin{array}{c}\text { Precip- } \\
\text { itation } \\
\text { (in.) } \\
\end{array}$ & $\begin{array}{c}\text { Cumu- } \\
\text { lative } \\
\text { precip- } \\
\text { itation } \\
\text { (in.) }\end{array}$ & $\begin{array}{c}\text { Precip- } \\
\text { itation } \\
(\mathrm{mm})\end{array}$ & $\begin{array}{c}\text { Cumu- } \\
\text { lative } \\
\text { precip- } \\
\text { itation } \\
(\mathrm{mm})\end{array}$ & $\begin{array}{c}\text { Precip- } \\
\text { itation } \\
\text { (in.) } \\
\end{array}$ & $\begin{array}{c}\text { Cumu- } \\
\text { lative } \\
\text { precip- } \\
\text { itation } \\
\text { (in.) }\end{array}$ & $\begin{array}{l}\text { Precip- } \\
\text { itation } \\
(\mathrm{mm})\end{array}$ & $\begin{array}{c}\text { Cumu- } \\
\text { lative } \\
\text { precip- } \\
\text { itation } \\
(\mathrm{mm})\end{array}$ & \\
\hline 933 & 0.00 & 19.45 & 0.00 & 494 & 0.00 & 5.99 & 0.00 & 152 & 0.00 & 5.52 & 0.00 & 140 & \\
\hline 934 & 0.06 & 19.51 & 1.52 & 495 & 0.00 & 5.99 & 0.00 & 152 & 0.00 & 5.52 & 0.00 & 140 & \\
\hline 935 & 0.00 & 19.51 & 0.00 & 495 & 0.00 & 5.99 & 0.00 & 152 & 0.00 & 5.52 & 0.00 & 140 & \\
\hline 936 & 0.00 & 19.51 & 0.00 & 495 & 0.01 & 6.00 & 0.25 & 152 & 0.00 & 5.52 & 0.00 & 140 & \\
\hline 937 & 0.03 & 19.54 & 0.76 & 496 & 0.30 & 6.30 & 7.62 & 160 & 0.04 & 5.56 & 1.02 & 141 & \\
\hline 938 & 0.46 & 20.00 & 11.68 & 508 & 0.00 & 6.30 & 0.00 & 160 & 0.59 & 6.15 & 14.99 & 156 & \\
\hline 939 & 0.00 & 20.00 & 0.00 & 508 & 0.00 & 6.30 & 0.00 & 160 & 0.00 & 6.15 & 0.00 & 156 & \\
\hline 940 & 0.00 & 20.00 & 0.00 & 508 & 0.00 & 6.30 & 0.00 & 160 & 0.00 & 6.15 & 0.00 & 156 & \\
\hline 941 & 0.00 & 20.00 & 0.00 & 508 & 0.00 & 6.30 & 0.00 & 160 & 0.00 & 6.15 & 0.00 & 156 & \\
\hline 942 & 0.00 & 20.00 & 0.00 & 508 & 0.00 & 6.30 & 0.00 & 160 & 0.00 & 6.15 & 0.00 & 156 & \\
\hline 943 & 0.00 & 20.00 & 0.00 & 508 & 0.00 & 6.30 & 0.00 & 160 & 0.00 & 6.15 & 0.00 & 156 & \\
\hline 944 & 0.00 & 20.00 & 0.00 & 508 & 0.00 & 6.30 & 0.00 & 160 & 0.00 & 6.15 & 0.00 & 156 & 1 August 2005 \\
\hline 945 & 0.01 & 20.01 & 0.25 & 508 & 0.01 & 6.31 & 0.25 & 160 & 0.00 & 6.15 & 0.00 & 156 & \\
\hline 946 & 0.46 & 20.47 & 11.68 & 520 & 0.00 & 6.31 & 0.00 & 160 & 0.00 & 6.15 & 0.00 & 156 & \\
\hline 947 & 0.04 & 20.51 & 1.02 & 521 & 0.02 & 6.33 & 0.51 & 161 & 0.00 & 6.15 & 0.00 & 156 & \\
\hline 948 & 0.20 & 20.71 & 5.08 & 526 & 0.02 & 6.35 & 0.51 & 161 & 0.04 & 6.19 & 1.02 & 157 & \\
\hline 949 & 0.02 & 20.73 & 0.51 & 526 & 0.01 & 6.36 & 0.25 & 162 & 0.00 & 6.19 & 0.00 & 157 & \\
\hline 950 & 0.00 & 20.73 & 0.00 & 526 & 0.00 & 6.36 & 0.00 & 162 & 0.00 & 6.19 & 0.00 & 157 & \\
\hline 951 & 0.00 & 20.73 & 0.00 & 526 & 0.00 & 6.36 & 0.00 & 162 & 0.00 & 6.19 & 0.00 & 157 & \\
\hline 952 & 0.00 & 20.73 & 0.00 & 526 & 0.03 & 6.39 & 0.76 & 162 & 0.00 & 6.19 & 0.00 & 157 & \\
\hline 953 & 0.01 & 20.74 & 0.25 & 527 & 0.07 & 6.46 & 1.78 & 164 & 0.06 & 6.25 & 1.52 & 159 & \\
\hline 954 & 0.11 & 20.85 & 2.79 & 529 & 0.03 & 6.49 & 0.76 & 165 & 0.04 & 6.29 & 1.02 & 160 & \\
\hline 955 & Trace & 20.85 & Trace & 530 & 0.01 & 6.50 & 0.25 & 165 & 0.00 & 6.29 & 0.00 & 160 & \\
\hline 956 & 0.00 & 20.85 & 0.00 & 530 & 0.00 & 6.50 & 0.00 & 165 & 0.01 & 6.30 & 0.25 & 160 & \\
\hline 957 & 0.01 & 20.86 & 0.25 & 530 & 0.00 & 6.50 & 0.00 & 165 & 0.00 & 6.30 & 0.00 & 160 & \\
\hline 958 & 0.00 & 20.86 & 0.00 & 530 & 0.00 & 6.50 & 0.00 & 165 & 0.00 & 6.30 & 0.00 & 160 & \\
\hline 959 & 0.10 & 20.96 & 2.54 & 532 & 0.26 & 6.76 & 6.60 & 172 & 0.05 & 6.35 & 1.27 & 161 & \\
\hline 960 & 0.76 & 21.72 & 19.30 & 552 & 0.12 & 6.88 & 3.05 & 175 & 0.04 & 6.39 & 1.02 & 162 & \\
\hline 961 & 0.22 & 21.94 & 5.59 & 557 & 0.00 & 6.88 & 0.00 & 175 & 0.00 & 6.39 & 0.00 & 162 & \\
\hline 962 & 0.00 & 21.94 & 0.00 & 557 & 0.04 & 6.92 & 1.02 & 176 & 0.00 & 6.39 & 0.00 & 162 & \\
\hline 963 & 0.50 & 22.44 & 12.70 & 570 & 0.00 & 6.92 & 0.00 & 176 & 0.00 & 6.39 & 0.00 & 162 & \\
\hline 964 & 0.00 & 22.44 & 0.00 & 570 & 0.00 & 6.92 & 0.00 & 176 & 0.00 & 6.39 & 0.00 & 162 & \\
\hline 965 & 0.01 & 22.45 & 0.25 & 570 & 0.00 & 6.92 & 0.00 & 176 & 0.00 & 6.39 & 0.00 & 162 & \\
\hline 966 & 0.02 & 22.47 & 0.51 & 571 & 0.00 & 6.92 & 0.00 & 176 & 0.00 & 6.39 & 0.00 & 162 & \\
\hline 967 & Trace & 22.47 & Trace & 571 & 0.00 & 6.92 & 0.00 & 176 & 0.05 & 6.44 & 1.27 & 164 & \\
\hline 968 & Trace & 22.48 & Trace & 571 & 0.03 & 6.95 & 0.76 & 177 & 0.00 & 6.44 & 0.00 & 164 & \\
\hline 969 & 0.43 & 22.91 & 10.92 & 582 & 0.00 & 6.95 & 0.00 & 177 & 0.01 & 6.45 & 0.25 & 164 & \\
\hline 970 & 0.02 & 22.93 & 0.51 & 582 & 0.00 & 6.95 & 0.00 & 177 & 0.00 & 6.45 & 0.00 & 164 & \\
\hline 971 & 0.00 & 22.93 & 0.00 & 582 & 0.00 & 6.95 & 0.00 & 177 & 0.00 & 6.45 & 0.00 & 164 & \\
\hline 972 & 0.00 & 22.93 & 0.00 & 582 & 0.00 & 6.95 & 0.00 & 177 & 0.00 & 6.45 & 0.00 & 164 & \\
\hline 973 & 0.00 & 22.93 & 0.00 & 582 & 0.00 & 6.95 & 0.00 & 177 & 0.00 & 6.45 & 0.00 & 164 & \\
\hline 974 & 0.00 & 22.93 & 0.00 & 582 & 0.00 & 6.95 & 0.00 & 177 & 0.00 & 6.45 & 0.00 & 164 & \\
\hline 975 & 0.00 & 22.93 & 0.00 & 582 & 0.00 & 6.95 & 0.00 & 177 & 0.01 & 6.46 & 0.25 & 164 & 1 September 2005 \\
\hline 976 & 0.00 & 22.93 & 0.00 & 582 & 0.00 & 6.95 & 0.00 & 177 & 0.00 & 6.46 & 0.00 & 164 & \\
\hline 977 & 0.00 & 22.93 & 0.00 & 582 & 0.00 & 6.95 & 0.00 & 177 & 0.00 & 6.46 & 0.00 & 164 & \\
\hline
\end{tabular}


Table 7. Daily and cumulative precipitation at the Montrose \#2 climate station, upper Elephant Skin Wash, and Candy Lane rain gages, southwestern Colorado.-Continued

[in., inch; mm, millimeter; md, missing data due to equipment malfunction or vandalism; MA, Montrose \#2 climate station; ES, upper Elephant Skin Wash; CL, Candy Lane]

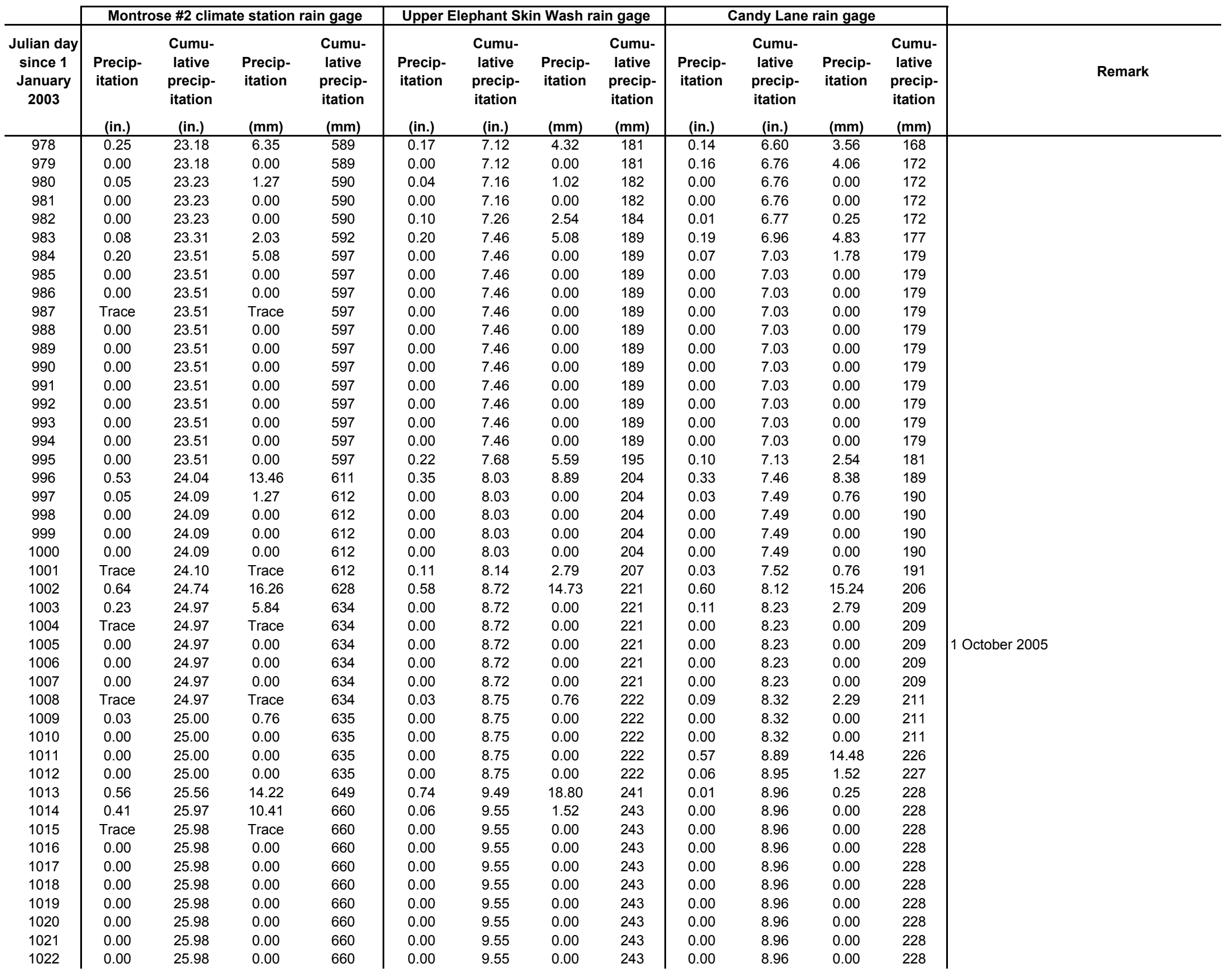


Table 7. Daily and cumulative precipitation at the Montrose \#2 climate station, upper Elephant Skin Wash, and Candy Lane rain gages, southwestern Colorado.-Continued

[in., inch; mm, millimeter; md, missing data due to equipment malfunction or vandalism; MA, Montrose \#2 climate station; ES, upper Elephant Skin Wash; CL, Candy Lane]

\begin{tabular}{|c|c|c|c|c|c|c|c|c|c|c|c|c|c|}
\hline \multirow[b]{2}{*}{$\begin{array}{c}\text { Julian day } \\
\text { since } 1 \\
\text { January } \\
2003\end{array}$} & \multicolumn{4}{|c|}{ Montrose \#2 climate station rain gage } & \multicolumn{4}{|c|}{ Upper Elephant Skin Wash rain gage } & \multicolumn{4}{|c|}{ Candy Lane rain gage } & \multirow[b]{2}{*}{ Remark } \\
\hline & $\begin{array}{l}\text { Precip- } \\
\text { itation } \\
\text { (in.) } \\
\end{array}$ & $\begin{array}{l}\text { Cumu- } \\
\text { lative } \\
\text { precip- } \\
\text { itation } \\
\text { (in.) }\end{array}$ & $\begin{array}{l}\text { Precip- } \\
\text { itation } \\
(\mathrm{mm})\end{array}$ & $\begin{array}{l}\text { Cumu- } \\
\text { lative } \\
\text { precip- } \\
\text { itation } \\
(\mathrm{mm})\end{array}$ & $\begin{array}{l}\text { Precip- } \\
\text { itation } \\
\text { (in.) } \\
\end{array}$ & $\begin{array}{l}\text { Cumu- } \\
\text { lative } \\
\text { precip- } \\
\text { itation } \\
\text { (in.) }\end{array}$ & $\begin{array}{l}\text { Precip- } \\
\text { itation } \\
(\mathrm{mm})\end{array}$ & $\begin{array}{l}\text { Cumu- } \\
\text { lative } \\
\text { precip- } \\
\text { itation } \\
(\mathrm{mm})\end{array}$ & $\begin{array}{l}\text { Precip- } \\
\text { itation } \\
\text { (in.) }\end{array}$ & $\begin{array}{l}\text { Cumu- } \\
\text { lative } \\
\text { precip- } \\
\text { itation } \\
\text { (in.) }\end{array}$ & $\begin{array}{l}\text { Precip- } \\
\text { itation } \\
(\mathrm{mm})\end{array}$ & $\begin{array}{c}\text { Cumu- } \\
\text { lative } \\
\text { precip- } \\
\text { itation } \\
(\mathrm{mm})\end{array}$ & \\
\hline 1023 & 0.27 & 26.25 & 6.86 & 667 & 0.13 & 9.68 & 3.30 & 246 & md & $\mathrm{md}$ & $\mathrm{md}$ & $\mathrm{md}$ & 19 October $2005, \mathrm{CL}$ rain gage pen fails to return \\
\hline 1024 & 0.00 & 26.25 & 0.00 & 667 & 0.00 & 9.68 & 0.00 & 246 & $\mathrm{md}$ & $\mathrm{md}$ & $\mathrm{md}$ & $\mathrm{md}$ & \\
\hline 1025 & 0.00 & 26.25 & 0.00 & 667 & 0.00 & 9.68 & 0.00 & 246 & $\mathrm{md}$ & $\mathrm{md}$ & $\mathrm{md}$ & md & \\
\hline 1026 & 0.00 & 26.25 & 0.00 & 667 & 0.00 & 9.68 & 0.00 & 246 & $\mathrm{md}$ & md & $\mathrm{md}$ & $\mathrm{md}$ & \\
\hline 1027 & 0.00 & 26.25 & 0.00 & 667 & 0.00 & 9.68 & 0.00 & 246 & md & md & $\mathrm{md}$ & md & \\
\hline 1028 & 0.00 & 26.25 & 0.00 & 667 & 0.00 & 9.68 & 0.00 & 246 & md & md & md & md & \\
\hline 1029 & 0.00 & 26.25 & 0.00 & 667 & 0.00 & 9.68 & 0.00 & 246 & $\mathrm{md}$ & md & md & md & \\
\hline 1030 & 0.00 & 26.25 & 0.00 & 667 & 0.00 & 9.68 & 0.00 & 246 & 0.00 & 9.68 & 0.00 & 246 & Equate $C L$ cumulative precpitation to $\mathrm{ES}$ \\
\hline 1031 & 0.00 & 26.25 & 0.00 & 667 & 0.00 & 9.68 & 0.00 & 246 & 0.00 & 9.68 & 0.00 & 246 & \\
\hline 1032 & 0.00 & 26.25 & 0.00 & 667 & 0.00 & 9.68 & 0.00 & 246 & 0.00 & 9.68 & 0.00 & 246 & \\
\hline 1033 & 0.00 & 26.25 & 0.00 & 667 & 0.01 & 9.69 & 0.25 & 246 & 0.05 & 9.73 & 1.27 & 247 & \\
\hline 1034 & 0.04 & 26.29 & 1.02 & 668 & 0.06 & 9.75 & 1.52 & 248 & 0.07 & 9.80 & 1.78 & 249 & \\
\hline 1035 & 0.13 & 26.42 & 3.30 & 671 & 0.00 & 9.75 & 0.00 & 248 & 0.00 & 9.80 & 0.00 & 249 & \\
\hline 1036 & 0.00 & 26.42 & 0.00 & 671 & 0.00 & 9.75 & 0.00 & 248 & 0.00 & 9.80 & 0.00 & 249 & 1 November 2005 \\
\hline 1037 & 0.00 & 26.42 & 0.00 & 671 & 0.00 & 9.75 & 0.00 & 248 & 0.00 & 9.80 & 0.00 & 249 & \\
\hline 1038 & 0.00 & 26.42 & 0.00 & 671 & 0.00 & 9.75 & 0.00 & 248 & 0.00 & 9.80 & 0.00 & 249 & \\
\hline 1039 & 0.00 & 26.42 & 0.00 & 671 & 0.00 & 9.75 & 0.00 & 248 & 0.00 & 9.80 & 0.00 & 249 & \\
\hline 1040 & 0.00 & 26.42 & 0.00 & 671 & 0.00 & 9.75 & 0.00 & 248 & 0.00 & 9.80 & 0.00 & 249 & \\
\hline 1041 & 0.00 & 26.42 & 0.00 & 671 & 0.00 & 9.75 & 0.00 & 248 & 0.00 & 9.80 & 0.00 & 249 & \\
\hline 1042 & 0.00 & 26.42 & 0.00 & 671 & 0.00 & 9.75 & 0.00 & 248 & 0.00 & 9.80 & 0.00 & 249 & \\
\hline 1043 & 0.00 & 26.42 & 0.00 & 671 & 0.00 & 9.75 & 0.00 & 248 & 0.00 & 9.80 & 0.00 & 249 & \\
\hline 1044 & 0.00 & 26.42 & 0.00 & 671 & 0.00 & 9.75 & 0.00 & 248 & 0.00 & 9.80 & 0.00 & 249 & \\
\hline 1045 & 0.00 & 26.42 & 0.00 & 671 & 0.00 & 9.75 & 0.00 & 248 & 0.00 & 9.80 & 0.00 & 249 & \\
\hline 1046 & 0.00 & 26.42 & 0.00 & 671 & 0.12 & 9.87 & 3.05 & 251 & 0.08 & 9.88 & 2.03 & 251 & \\
\hline 1047 & 0.15 & 26.57 & 3.81 & 675 & 0.01 & 9.88 & 0.25 & 251 & 0.00 & 9.88 & 0.00 & 251 & \\
\hline 1048 & 0.00 & 26.57 & 0.00 & 675 & 0.00 & 9.88 & 0.00 & 251 & 0.00 & 9.88 & 0.00 & 251 & \\
\hline 1049 & 0.00 & 26.57 & 0.00 & 675 & 0.07 & 9.95 & 1.78 & 253 & 0.06 & 9.94 & 1.52 & 252 & \\
\hline 1050 & 0.12 & 26.69 & 3.05 & 678 & 0.00 & 9.95 & 0.00 & 253 & 0.00 & 9.94 & 0.00 & 252 & \\
\hline 1051 & 0.00 & 26.69 & 0.00 & 678 & 0.00 & 9.95 & 0.00 & 253 & 0.00 & 9.94 & 0.00 & 252 & \\
\hline 1052 & 0.00 & 26.69 & 0.00 & 678 & 0.00 & 9.95 & 0.00 & 253 & 0.00 & 9.94 & 0.00 & 252 & \\
\hline 1053 & 0.00 & 26.69 & 0.00 & 678 & 0.00 & 9.95 & 0.00 & 253 & 0.00 & 9.94 & 0.00 & 252 & \\
\hline 1054 & 0.00 & 26.69 & 0.00 & 678 & 0.00 & 9.95 & 0.00 & 253 & 0.00 & 9.94 & 0.00 & 252 & \\
\hline 1055 & 0.00 & 26.69 & 0.00 & 678 & 0.00 & 9.95 & 0.00 & 253 & 0.00 & 9.94 & 0.00 & 252 & \\
\hline 1056 & 0.00 & 26.69 & 0.00 & 678 & 0.00 & 9.95 & 0.00 & 253 & 0.00 & 9.94 & 0.00 & 252 & \\
\hline 1057 & 0.00 & 26.69 & 0.00 & 678 & 0.00 & 9.95 & 0.00 & 253 & 0.00 & 9.94 & 0.00 & 252 & \\
\hline 1058 & 0.00 & 26.69 & 0.00 & 678 & 0.00 & 9.95 & 0.00 & 253 & 0.00 & 9.94 & 0.00 & 252 & \\
\hline 1059 & 0.00 & 26.69 & 0.00 & 678 & 0.00 & 9.95 & 0.00 & 253 & 0.00 & 9.94 & 0.00 & 252 & \\
\hline 1060 & 0.00 & 26.69 & 0.00 & 678 & 0.00 & 9.95 & 0.00 & 253 & 0.00 & 9.94 & 0.00 & 252 & \\
\hline 1061 & 0.00 & 26.69 & 0.00 & 678 & 0.00 & 9.95 & 0.00 & 253 & 0.00 & 9.94 & 0.00 & 252 & \\
\hline 1062 & 0.00 & 26.69 & 0.00 & 678 & 0.00 & 9.95 & 0.00 & 253 & 0.00 & 9.94 & 0.00 & 252 & \\
\hline 1063 & 0.00 & 26.69 & 0.00 & 678 & 0.00 & 9.95 & 0.00 & 253 & 0.00 & 9.94 & 0.00 & 252 & \\
\hline 1064 & 0.00 & 26.69 & 0.00 & 678 & 0.39 & 10.34 & 9.91 & 263 & 0.00 & 9.94 & 0.00 & 252 & \\
\hline 1065 & 0.00 & 26.69 & 0.00 & 678 & 0.02 & 10.36 & 0.51 & 263 & 0.00 & 9.94 & 0.00 & 252 & \\
\hline 1066 & 0.00 & 26.69 & 0.00 & 678 & 0.02 & 10.38 & 0.51 & 264 & 0.00 & 9.94 & 0.00 & 252 & 1 December 2005 \\
\hline 1067 & 0.00 & 26.69 & 0.00 & 678 & 0.00 & 10.38 & 0.00 & 264 & 0.17 & 10.11 & 4.32 & 257 & \\
\hline
\end{tabular}


Table 7. Daily and cumulative precipitation at the Montrose \#2 climate station, upper Elephant Skin Wash, and Candy Lane rain gages, southwestern Colorado.-Continued

[in., inch; mm, millimeter; md, missing data due to equipment malfunction or vandalism; MA, Montrose \#2 climate station; ES, upper Elephant Skin Wash; CL, Candy Lane]

\begin{tabular}{|c|c|c|c|c|c|c|c|c|c|c|c|c|c|}
\hline \multirow[b]{2}{*}{$\begin{array}{c}\text { Julian day } \\
\text { since } 1 \\
\text { January } \\
2003\end{array}$} & \multicolumn{4}{|c|}{ Montrose \#2 climate station rain gage } & \multicolumn{4}{|c|}{ Upper Elephant Skin Wash rain gage } & \multicolumn{4}{|c|}{ Candy Lane rain gage } & \multirow[b]{2}{*}{ Remark } \\
\hline & $\begin{array}{l}\text { Precip- } \\
\text { itation } \\
\text { (in.) } \\
\end{array}$ & $\begin{array}{c}\text { Cumu- } \\
\text { lative } \\
\text { precip- } \\
\text { itation } \\
\text { (in.) }\end{array}$ & $\begin{array}{l}\text { Precip- } \\
\text { itation } \\
(\mathrm{mm}) \\
\end{array}$ & $\begin{array}{l}\text { Cumu- } \\
\text { lative } \\
\text { precip- } \\
\text { itation } \\
(\mathrm{mm})\end{array}$ & $\begin{array}{c}\text { Precip- } \\
\text { itation } \\
\text { (in.) } \\
\end{array}$ & $\begin{array}{c}\text { Cumu- } \\
\text { lative } \\
\text { precip- } \\
\text { itation } \\
\text { (in.) }\end{array}$ & $\begin{array}{l}\text { Precip- } \\
\text { itation } \\
(\mathrm{mm})\end{array}$ & $\begin{array}{c}\text { Cumu- } \\
\text { lative } \\
\text { precip- } \\
\text { itation } \\
(\mathrm{mm})\end{array}$ & 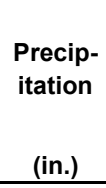 & $\begin{array}{c}\text { Cumu- } \\
\text { lative } \\
\text { precip- } \\
\text { itation } \\
\text { (in.) }\end{array}$ & $\begin{array}{l}\text { Precip- } \\
\text { itation } \\
(\mathrm{mm})\end{array}$ & $\begin{array}{c}\text { Cumu- } \\
\text { lative } \\
\text { precip- } \\
\text { itation } \\
(\mathrm{mm})\end{array}$ & \\
\hline 1068 & 0.28 & 26.97 & 7.11 & 685 & 0.00 & 10.38 & 0.00 & 264 & 0.04 & 10.15 & 1.02 & 258 & \\
\hline 1069 & 0.04 & 27.01 & 1.02 & 686 & 0.00 & 10.38 & 0.00 & 264 & 0.00 & 10.15 & 0.00 & 258 & \\
\hline 1070 & Trace & 27.01 & Trace & 686 & 0.00 & 10.38 & 0.00 & 264 & 0.00 & 10.15 & 0.00 & 258 & \\
\hline 1072 & 0.00 & 27.02 & 0.00 & 686 & 0.03 & 10.41 & 0.76 & 264 & 0.01 & 10.16 & 0.25 & 258 & \\
\hline 1073 & 0.00 & 27.02 & 0.00 & 686 & 0.00 & 10.41 & 0.00 & 264 & 0.01 & 10.17 & 0.25 & 258 & \\
\hline 1074 & 0.00 & 27.02 & 0.00 & 686 & 0.00 & 10.41 & 0.00 & 264 & 0.00 & 10.17 & 0.00 & 258 & \\
\hline 1075 & 0.00 & 27.02 & 0.00 & 686 & 0.00 & 10.41 & 0.00 & 264 & 0.00 & 10.17 & 0.00 & 258 & \\
\hline 1076 & 0.00 & 27.02 & 0.00 & 686 & 0.00 & 10.41 & 0.00 & 264 & 0.00 & 10.17 & 0.00 & 258 & \\
\hline 1077 & 0.00 & 27.02 & 0.00 & 686 & 0.00 & 10.41 & 0.00 & 264 & 0.00 & 10.17 & 0.00 & 258 & \\
\hline 1078 & Trace & 27.02 & Trace & 686 & 0.04 & 10.45 & 1.02 & 265 & 0.04 & 10.21 & 1.02 & 259 & \\
\hline 1079 & 0.00 & 27.02 & 0.00 & 686 & 0.00 & 10.45 & 0.00 & 265 & 0.02 & 10.23 & 0.51 & 260 & \\
\hline 1080 & 0.00 & 27.02 & 0.00 & 686 & 0.00 & 10.45 & 0.00 & 265 & 0.00 & 10.23 & 0.00 & 260 & \\
\hline 1081 & 0.00 & 27.02 & 0.00 & 686 & 0.00 & 10.45 & 0.00 & 265 & 0.00 & 10.23 & 0.00 & 260 & \\
\hline 1084 & 0.02 & 27.04 & 0.51 & 687 & 0.00 & 10.45 & 0.00 & 265 & 0.04 & 10.28 & 1.02 & 261 & \\
\hline 1085 & Trace & 27.04 & Trace & 687 & 0.00 & 10.45 & 0.00 & 265 & 0.00 & 10.28 & 0.00 & 261 & \\
\hline 1086 & 0.00 & 27.04 & 0.00 & 687 & 0.10 & 10.55 & 2.54 & 268 & 0.00 & 10.28 & 0.00 & 261 & \\
\hline 1087 & 0.00 & 27.04 & 0.00 & 687 & 0.00 & 10.55 & 0.00 & 268 & 0.00 & 10.28 & 0.00 & 261 & \\
\hline 1088 & 0.00 & 27.04 & 0.00 & 687 & 0.02 & 10.57 & 0.51 & 268 & 0.00 & 10.28 & 0.00 & 261 & \\
\hline 1089 & 0.00 & 27.04 & 0.00 & 687 & 0.00 & 10.57 & 0.00 & 268 & 0.00 & 10.28 & 0.00 & 261 & \\
\hline 1090 & 0.00 & 27.04 & 0.00 & 687 & 0.01 & 10.58 & 0.25 & 269 & 0.00 & 10.28 & 0.00 & 261 & \\
\hline 1091 & 0.00 & 27.04 & 0.00 & 687 & 0.06 & 10.64 & 1.52 & 270 & 0.00 & 10.28 & 0.00 & 261 & \\
\hline 1092 & 0.02 & 27.06 & 0.51 & 687 & 0.00 & 10.64 & 0.00 & 270 & 0.05 & 10.33 & 1.27 & 262 & \\
\hline 1093 & 0.00 & 27.06 & 0.00 & 687 & 0.02 & 10.66 & 0.51 & 271 & 0.00 & 10.33 & 0.00 & 262 & \\
\hline 1094 & 0.00 & 27.06 & 0.00 & 687 & 0.00 & 10.66 & 0.00 & 271 & 0.02 & 10.35 & 0.51 & 263 & \\
\hline 1095 & 0.00 & 27.06 & 0.00 & 687 & 0.00 & 10.66 & 0.00 & 271 & 0.00 & 10.35 & 0.00 & 263 & \\
\hline 1096 & 0.00 & 27.06 & 0.00 & 687 & 0.00 & 10.66 & 0.00 & 271 & 0.02 & 10.37 & 0.51 & 263 & \\
\hline 1097 & Trace & 27.07 & Trace & 688 & 0.00 & 10.66 & 0.00 & 271 & 0.03 & 10.40 & 0.76 & 264 & 1 January 2006 \\
\hline 1098 & 0.00 & 27.07 & 0.00 & 688 & 0.00 & 10.66 & 0.00 & 271 & 0.00 & 10.40 & 0.00 & 264 & \\
\hline 1099 & Trace & 27.07 & Trace & 688 & 0.00 & 10.66 & 0.00 & 271 & 0.00 & 10.40 & 0.00 & 264 & \\
\hline 1100 & 0.00 & 27.07 & 0.00 & 688 & 0.00 & 10.66 & 0.00 & 271 & 0.00 & 10.40 & 0.00 & 264 & \\
\hline 1101 & 0.00 & 27.07 & 0.00 & 688 & 0.00 & 10.66 & 0.00 & 271 & 0.00 & 10.40 & 0.00 & 264 & \\
\hline 1102 & 0.00 & 27.07 & 0.00 & 688 & 0.00 & 10.66 & 0.00 & 271 & 0.00 & 10.40 & 0.00 & 264 & \\
\hline 1103 & 0.00 & 27.07 & 0.00 & 688 & 0.00 & 10.66 & 0.00 & 271 & 0.00 & 10.40 & 0.00 & 264 & \\
\hline 1106 & 0.00 & 27.07 & 0.00 & 688 & 0.29 & 10.98 & 7.37 & 279 & 0.00 & 10.40 & 0.00 & 264 & \\
\hline 1107 & 0.00 & 27.07 & 0.00 & 688 & 0.00 & 10.98 & 0.00 & 279 & 0.00 & 10.40 & 0.00 & 264 & \\
\hline 1108 & 0.00 & 27.07 & 0.00 & 688 & 0.00 & 10.98 & 0.00 & 279 & 0.00 & 10.40 & 0.00 & 264 & \\
\hline 1109 & 0.00 & 27.07 & 0.00 & 688 & 0.07 & 11.05 & 1.78 & 281 & 0.00 & 10.40 & 0.00 & 264 & \\
\hline 1110 & 0.00 & 27.07 & 0.00 & 688 & 0.02 & 11.07 & 0.51 & 281 & 0.00 & 10.40 & 0.00 & 264 & \\
\hline 1111 & 0.00 & 27.07 & 0.00 & 688 & 0.00 & 11.07 & 0.00 & 281 & 0.02 & 10.42 & 0.51 & 265 & \\
\hline 1112 & 0.22 & 27.29 & 5.59 & 693 & 0.00 & 11.07 & 0.00 & 281 & 0.27 & 10.69 & 6.86 & 272 & \\
\hline
\end{tabular}


Table 7. Daily and cumulative precipitation at the Montrose \#2 climate station, upper Elephant Skin Wash, and Candy Lane rain gages, southwestern Colorado.-Continued

[in., inch; mm, millimeter; md, missing data due to equipment malfunction or vandalism; MA, Montrose \#2 climate station; ES, upper Elephant Skin Wash; CL, Candy Lane]

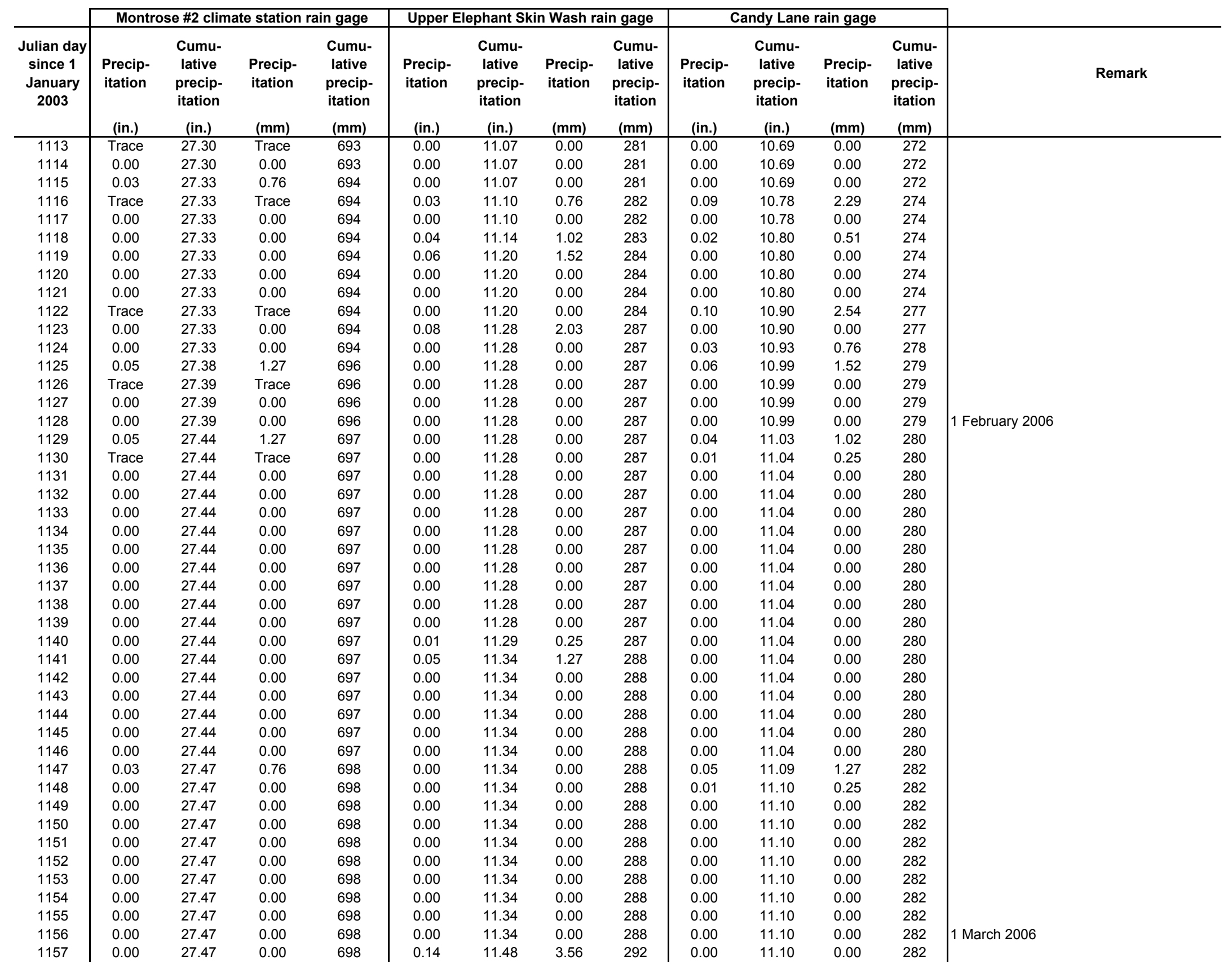


Table 7. Daily and cumulative precipitation at the Montrose \#2 climate station, upper Elephant Skin Wash, and Candy Lane rain gages, southwestern

[in., inch; mm, millimeter; md, missing data due to equipment malfunction or vandalism; MA, Montrose \#2 climate station; ES, upper Elephant Skin Wash; CL, Candy Lane]

\begin{tabular}{|c|c|c|c|c|c|c|c|c|c|c|c|c|c|}
\hline \multirow[b]{2}{*}{$\begin{array}{c}\text { Julian day } \\
\text { since } 1 \\
\text { January } \\
2003\end{array}$} & \multicolumn{4}{|c|}{ Montrose \#2 climate station rain gage } & \multicolumn{4}{|c|}{ Upper Elephant Skin Wash rain gage } & \multicolumn{4}{|c|}{ Candy Lane rain gage } & \multirow[b]{2}{*}{ Remark } \\
\hline & $\begin{array}{l}\text { Precip- } \\
\text { itation } \\
\text { (in.) }\end{array}$ & $\begin{array}{l}\text { Cumu- } \\
\text { lative } \\
\text { precip- } \\
\text { itation } \\
\text { (in.) }\end{array}$ & $\begin{array}{l}\text { Precip- } \\
\text { itation } \\
(\mathrm{mm})\end{array}$ & $\begin{array}{l}\text { Cumu- } \\
\text { lative } \\
\text { precip- } \\
\text { itation } \\
(\mathrm{mm})\end{array}$ & $\begin{array}{l}\text { Precip- } \\
\text { itation } \\
\text { (in.) }\end{array}$ & $\begin{array}{l}\text { Cumu- } \\
\text { lative } \\
\text { precip- } \\
\text { itation } \\
\text { (in.) }\end{array}$ & $\begin{array}{l}\text { Precip- } \\
\text { itation }\end{array}$ & $\begin{array}{l}\text { Cumu- } \\
\text { lative } \\
\text { precip- } \\
\text { itation } \\
(\mathrm{mm})\end{array}$ & $\begin{array}{l}\text { Precip- } \\
\text { itation }\end{array}$ & $\begin{array}{l}\text { Cumu- } \\
\text { lative } \\
\text { precip- } \\
\text { itation } \\
\text { (in.) }\end{array}$ & $\begin{array}{l}\text { Precip- } \\
\text { itation }\end{array}$ & $\begin{array}{l}\text { Cumu- } \\
\text { lative } \\
\text { precip- } \\
\text { itation } \\
(\mathrm{mm})\end{array}$ & \\
\hline 1158 & 0.00 & 27.47 & 0.00 & 698 & 0.00 & 11.48 & 0.00 & 292 & 0.01 & 11.11 & 0.25 & 282 & \\
\hline 1159 & 0.00 & 27.47 & 0.00 & 698 & 0.25 & 11.73 & 6.35 & 298 & 0.00 & 11.11 & 0.00 & 282 & 4 March $2006, C L$ rain gage shot \\
\hline 1160 & 0.00 & 27.47 & 0.00 & 698 & 0.19 & 11.92 & 4.83 & 303 & $\mathrm{md}$ & md & $\mathrm{md}$ & md & \\
\hline 1161 & 0.00 & 27.47 & 0.00 & 698 & 0.06 & 11.98 & 1.52 & 304 & md & md & md & md & \\
\hline 1162 & 0.00 & 27.47 & 0.00 & 698 & 0.02 & 12.00 & 0.51 & 305 & $\mathrm{md}$ & $\mathrm{md}$ & $\mathrm{md}$ & md & \\
\hline 1163 & 0.02 & 27.49 & 0.51 & 698 & 0.00 & 12.00 & 0.00 & 305 & $\mathrm{md}$ & $\mathrm{md}$ & $\mathrm{md}$ & $\mathrm{md}$ & \\
\hline 1164 & 0.03 & 27.52 & 0.76 & 699 & 0.00 & 12.00 & 0.00 & 305 & $\mathrm{md}$ & $\mathrm{md}$ & $\mathrm{md}$ & md & \\
\hline 1165 & 0.14 & 27.66 & 3.56 & 703 & 0.00 & 12.00 & 0.00 & 305 & $\mathrm{md}$ & $\mathrm{md}$ & $\mathrm{md}$ & $\mathrm{md}$ & \\
\hline 1166 & 0.12 & 27.78 & 3.05 & 706 & 0.00 & 12.00 & 0.00 & 305 & $\mathrm{md}$ & $\mathrm{md}$ & $\mathrm{md}$ & $\mathrm{md}$ & \\
\hline 1167 & Trace & 27.79 & Trace & 706 & 0.07 & 12.07 & 1.78 & 307 & $\mathrm{md}$ & $\mathrm{md}$ & $\mathrm{md}$ & $\mathrm{md}$ & \\
\hline 1168 & 0.02 & 27.81 & 0.51 & 706 & 0.00 & 12.07 & 0.00 & 307 & $\mathrm{md}$ & $\mathrm{md}$ & $\mathrm{md}$ & $\mathrm{md}$ & \\
\hline 1169 & 0.00 & 27.81 & 0.00 & 706 & 0.00 & 12.07 & 0.00 & 307 & $\mathrm{md}$ & $\mathrm{md}$ & $\mathrm{md}$ & $\mathrm{md}$ & \\
\hline 1170 & 0.00 & 27.81 & 0.00 & 706 & 0.00 & 12.07 & 0.00 & 307 & $\mathrm{md}$ & $\mathrm{md}$ & md & md & \\
\hline 1171 & 0.00 & 27.81 & 0.00 & 706 & 0.00 & 12.07 & 0.00 & 307 & $\mathrm{md}$ & $\mathrm{md}$ & $\mathrm{md}$ & $\mathrm{md}$ & \\
\hline 1172 & 0.00 & 27.81 & 0.00 & 706 & 0.00 & 12.07 & 0.00 & 307 & $\mathrm{md}$ & $\mathrm{md}$ & $\mathrm{md}$ & $\mathrm{md}$ & \\
\hline 1174 & 0.03 & 27.84 & 0.76 & 707 & 0.00 & 12.07 & 0.00 & 307 & $\mathrm{md}$ & $\mathrm{md}$ & $\mathrm{md}$ & $\mathrm{md}$ & \\
\hline 1175 & 0.00 & 27.84 & 0.00 & 707 & 0.00 & 12.07 & 0.00 & 307 & $\mathrm{md}$ & $\mathrm{md}$ & $\mathrm{md}$ & $\mathrm{md}$ & \\
\hline 1176 & 0.00 & 27.84 & 0.00 & 707 & 0.00 & 12.07 & 0.00 & 307 & $\mathrm{md}$ & $\mathrm{md}$ & $\mathrm{md}$ & $\mathrm{md}$ & \\
\hline 1177 & 0.00 & 27.84 & 0.00 & 707 & 0.00 & 12.07 & 0.00 & 307 & $\mathrm{md}$ & $\mathrm{md}$ & $\mathrm{md}$ & $\mathrm{md}$ & \\
\hline 1178 & 0.00 & 27.84 & 0.00 & 707 & 0.02 & 12.09 & 0.51 & 307 & $\mathrm{md}$ & $\mathrm{md}$ & $\mathrm{md}$ & $\mathrm{md}$ & \\
\hline 1179 & 0.00 & 27.84 & 0.00 & 707 & 0.16 & 12.25 & 4.06 & 311 & $\mathrm{md}$ & md & $\mathrm{md}$ & $\mathrm{md}$ & \\
\hline 1180 & 0.00 & 27.84 & 0.00 & 707 & 0.00 & 12.25 & 0.00 & 311 & $\mathrm{md}$ & $\mathrm{md}$ & $\mathrm{md}$ & $\mathrm{md}$ & \\
\hline 1181 & 0.00 & 27.84 & 0.00 & 707 & 0.00 & 12.25 & 0.00 & 311 & $\mathrm{md}$ & $\mathrm{md}$ & $\mathrm{md}$ & $\mathrm{md}$ & \\
\hline 1182 & 0.00 & 27.84 & 0.00 & 707 & 0.05 & 12.30 & 1.27 & 312 & $\mathrm{md}$ & $\mathrm{md}$ & $\mathrm{md}$ & $\mathrm{md}$ & \\
\hline 1183 & 0.00 & 27.84 & 0.00 & 707 & 0.00 & 12.30 & 0.00 & 312 & $\mathrm{md}$ & $\mathrm{md}$ & $\mathrm{md}$ & $\mathrm{md}$ & \\
\hline 1184 & 0.00 & 27.84 & 0.00 & 707 & 0.00 & 12.30 & 0.00 & 312 & $\mathrm{md}$ & $\mathrm{md}$ & $\mathrm{md}$ & $\mathrm{md}$ & \\
\hline 1185 & 0.21 & 28.05 & 5.33 & 712 & 0.00 & 12.30 & 0.00 & 312 & $\mathrm{md}$ & $\mathrm{md}$ & $\mathrm{md}$ & $\mathrm{md}$ & \\
\hline 1186 & 0.00 & 28.05 & 0.00 & 712 & 0.06 & 12.36 & 1.52 & 314 & $\mathrm{md}$ & $\mathrm{md}$ & $\mathrm{md}$ & $\mathrm{md}$ & \\
\hline 1187 & 0.00 & 28.05 & 0.00 & 712 & 0.00 & 12.36 & 0.00 & 314 & $\mathrm{md}$ & $\mathrm{md}$ & $\mathrm{md}$ & $\mathrm{md}$ & 1 April 2006 \\
\hline 1188 & 0.03 & 28.08 & 0.76 & 713 & 0.00 & 12.36 & 0.00 & 314 & $\mathrm{md}$ & md & $\mathrm{md}$ & md & \\
\hline 1189 & 0.00 & 28.08 & 0.00 & 713 & 0.00 & 12.36 & 0.00 & 314 & $\mathrm{md}$ & $\mathrm{md}$ & $\mathrm{md}$ & $\mathrm{md}$ & \\
\hline 1190 & 0.00 & 28.08 & 0.00 & 713 & 0.00 & 12.36 & 0.00 & 314 & $\mathrm{md}$ & $\mathrm{md}$ & $\mathrm{md}$ & $\mathrm{md}$ & \\
\hline 1191 & 0.00 & 28.08 & 0.00 & 713 & 0.00 & 12.36 & 0.00 & 314 & $\mathrm{md}$ & $\mathrm{md}$ & $\mathrm{md}$ & $\mathrm{md}$ & \\
\hline 1192 & 0.01 & 28.09 & 0.25 & 713 & 0.00 & 12.36 & 0.00 & 314 & $\mathrm{md}$ & $\mathrm{md}$ & $\mathrm{md}$ & $\mathrm{md}$ & \\
\hline 1193 & 0.00 & 28.09 & 0.00 & 713 & 0.00 & 12.36 & 0.00 & 314 & $\mathrm{md}$ & $\mathrm{md}$ & $\mathrm{md}$ & $\mathrm{md}$ & \\
\hline 1197 & 0.00 & 28.09 & 0.00 & 713 & 0.00 & 12.39 & 0.00 & 315 & $\mathrm{md}$ & $\mathrm{md}$ & $\mathrm{md}$ & $\mathrm{md}$ & \\
\hline 1198 & 0.00 & 28.09 & 0.00 & 713 & 0.00 & 12.39 & 0.00 & 315 & $\mathrm{md}$ & $\mathrm{md}$ & $\mathrm{md}$ & $\mathrm{md}$ & \\
\hline 1199 & 0.00 & 28.09 & 0.00 & 713 & 0.00 & 12.39 & 0.00 & 315 & $\mathrm{md}$ & $\mathrm{md}$ & $\mathrm{md}$ & $\mathrm{md}$ & \\
\hline 1200 & 0.00 & 28.09 & 0.00 & 713 & 0.00 & 12.39 & 0.00 & 315 & $\mathrm{md}$ & $\mathrm{md}$ & $\mathrm{md}$ & $\mathrm{md}$ & \\
\hline 1201 & 0.03 & 28.12 & 0.76 & 714 & 0.00 & 12.39 & 0.00 & 315 & $\mathrm{md}$ & $\mathrm{md}$ & $\mathrm{md}$ & $\mathrm{md}$ & \\
\hline 1202 & 0.00 & 28.12 & 0.00 & 714 & 0.00 & 12.39 & 0.00 & 315 & $\mathrm{md}$ & $\mathrm{md}$ & $\mathrm{md}$ & $\mathrm{md}$ & \\
\hline
\end{tabular}


Table 7. Daily and cumulative precipitation at the Montrose \#2 climate station, upper Elephant Skin Wash, and Candy Lane rain gages, southwestern Colorado.-Continued

[in., inch; mm, millimeter; md, missing data due to equipment malfunction or vandalism; MA, Montrose \#2 climate station; ES, upper Elephant Skin Wash; CL, Candy Lane]

\begin{tabular}{|c|c|c|c|c|c|c|c|c|c|c|c|c|c|}
\hline \multirow[b]{2}{*}{$\begin{array}{c}\text { Julian day } \\
\text { since } 1 \\
\text { January } \\
2003\end{array}$} & \multicolumn{4}{|c|}{ Montrose \#2 climate station rain gage } & \multicolumn{4}{|c|}{ Upper Elephant Skin Wash rain gage } & \multicolumn{4}{|c|}{ Candy Lane rain gage } & \multirow[b]{2}{*}{ Remark } \\
\hline & $\begin{array}{l}\text { Precip- } \\
\text { itation } \\
\text { (in.) } \\
\end{array}$ & $\begin{array}{l}\text { Cumu- } \\
\text { lative } \\
\text { precip- } \\
\text { itation } \\
\text { (in.) }\end{array}$ & $\begin{array}{l}\text { Precip- } \\
\text { itation } \\
(\mathrm{mm})\end{array}$ & $\begin{array}{l}\text { Cumu- } \\
\text { lative } \\
\text { precip- } \\
\text { itation } \\
(\mathrm{mm})\end{array}$ & $\begin{array}{l}\text { Precip- } \\
\text { itation } \\
\text { (in.) } \\
\end{array}$ & $\begin{array}{l}\text { Cumu- } \\
\text { lative } \\
\text { precip- } \\
\text { itation } \\
\text { (in.) }\end{array}$ & $\begin{array}{l}\text { Precip- } \\
\text { itation } \\
(\mathrm{mm})\end{array}$ & $\begin{array}{l}\text { Cumu- } \\
\text { lative } \\
\text { precip- } \\
\text { itation } \\
(\mathrm{mm})\end{array}$ & $\begin{array}{c}\text { Precip- } \\
\text { itation } \\
\text { (in.) }\end{array}$ & $\begin{array}{c}\text { Cumu- } \\
\text { lative } \\
\text { precip- } \\
\text { itation } \\
\text { (in.) }\end{array}$ & $\begin{array}{l}\text { Precip- } \\
\text { itation } \\
(\mathrm{mm})\end{array}$ & $\begin{array}{c}\text { Cumu- } \\
\text { lative } \\
\text { precip- } \\
\text { itation } \\
(\mathrm{mm})\end{array}$ & \\
\hline 1203 & 0.00 & 28.12 & 0.00 & 714 & 0.00 & 12.39 & 0.00 & 315 & $\mathrm{md}$ & $\mathrm{md}$ & $\mathrm{md}$ & $\mathrm{md}$ & \\
\hline 1204 & 0.00 & 28.12 & 0.00 & 714 & 0.00 & 12.39 & 0.00 & 315 & $\mathrm{md}$ & $\mathrm{md}$ & $\mathrm{md}$ & $\mathrm{md}$ & \\
\hline 1205 & 0.00 & 28.12 & 0.00 & 714 & 0.00 & 12.39 & 0.00 & 315 & $\mathrm{md}$ & $\mathrm{md}$ & $\mathrm{md}$ & $\mathrm{md}$ & \\
\hline 1206 & 0.00 & 28.12 & 0.00 & 714 & 0.00 & 12.39 & 0.00 & 315 & $\mathrm{md}$ & $\mathrm{md}$ & $\mathrm{md}$ & $\mathrm{md}$ & \\
\hline 1207 & 0.00 & 28.12 & 0.00 & 714 & 0.00 & 12.39 & 0.00 & 315 & $\mathrm{md}$ & $\mathrm{md}$ & $\mathrm{md}$ & $\mathrm{md}$ & \\
\hline 1208 & 0.00 & 28.12 & 0.00 & 714 & 0.00 & 12.39 & 0.00 & 315 & $\mathrm{md}$ & $\mathrm{md}$ & $\mathrm{md}$ & $\mathrm{md}$ & \\
\hline 1209 & 0.00 & 28.12 & 0.00 & 714 & 0.00 & 12.39 & 0.00 & 315 & $\mathrm{md}$ & $\mathrm{md}$ & $\mathrm{md}$ & $\mathrm{md}$ & \\
\hline 1210 & 0.00 & 28.12 & 0.00 & 714 & 0.00 & 12.39 & 0.00 & 315 & $\mathrm{md}$ & $\mathrm{md}$ & $\mathrm{md}$ & $\mathrm{md}$ & \\
\hline 1211 & 0.00 & 28.12 & 0.00 & 714 & 0.00 & 12.39 & 0.00 & 315 & $\mathrm{md}$ & $\mathrm{md}$ & $\mathrm{md}$ & $\mathrm{md}$ & 25 April $2006 \mathrm{CL}$ rain gage discontinued \\
\hline 1212 & 0.00 & 28.12 & 0.00 & 714 & 0.00 & 12.39 & 0.00 & 315 & $\mathrm{md}$ & $\mathrm{md}$ & md & $\mathrm{md}$ & 26 April 2006 ES rain gage discontinued \\
\hline
\end{tabular}




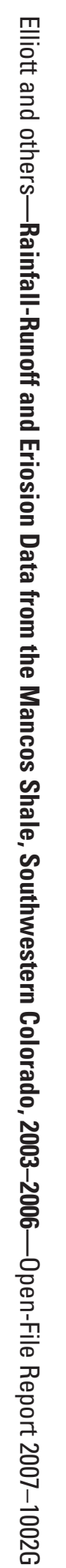

